\title{
PSYCHOPHYSICAL SUPERVENIENCE
}

\author{
Giovanna Enrica Hendel
}

Thesis submitted for the degree of $\mathrm{PhD}$

University College London

May 1999 
ProQuest Number: 10608926

All rights reserved

INFORMATION TO ALL USERS

The quality of this reproduction is dependent upon the quality of the copy submitted.

In the unlikely event that the author did not send a complete manuscript and there are missing pages, these will be noted. Also, if material had to be removed, a note will indicate the deletion.

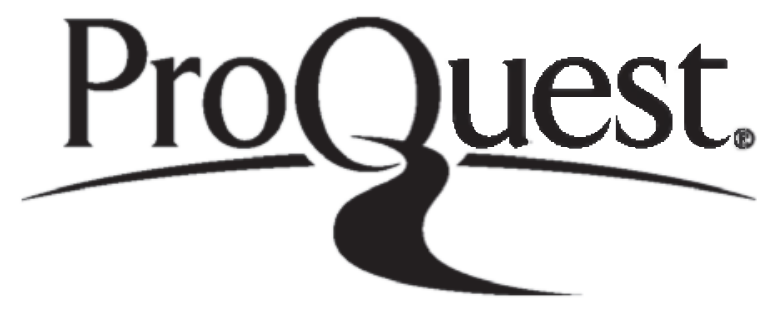

ProQuest 10608926

Published by ProQuest LLC (2017). Copyright of the Dissertation is held by the Author.

All rights reserved.

This work is protected against unauthorized copying under Title 17, United States Code Microform Edition @ ProQuest LLC.

ProQuest LLC.

789 East Eisenhower Parkway

P.O. Box 1346

Ann Arbor, Ml $48106-1346$ 


\begin{abstract}
I argue that, contrary to what is believed today by many philosophers, a supervenience claim can be quite strong (i.e. it can entail many further claims). To this extent, claims of psychophysical supervenience can be used to capture some possible positions with respect to the Mind-Body problem. In some cases, however, the strength of supervenience claims depends on adopting views which are not congenial to all. After considering the variety of forms of supervenience, and establishing what - in my opinion - are the important distinctions, I put forward and defend the thesis (Th) that psychophysical supervenience in its full generality can be satisfactorily supported if and only if one is willing to make any of some substantial assumptions (the 'Assumptions') about the nature of mental and physical properties. Given the nature of the Assumptions, (Th) adds potential strength to supervenience claims. I then argue for the idea of supervenience as a potentially strong thesis by showing that (a) psychophysical supervenience can yield reduction under certain understandings of 'reduction'; (b) a claim of psychophysical supervenience can, relatedly, correspond to a position which is inconsistent with some significant positions, such as that of Davidson and that of the Emergentist, in the debate on the MindBody problem; (c) some of the requirements which are commonly associated with the notion of dependence can be fulfilled on the basis of supervenience alone, and those which can't do not necessarily threaten the view that at least some forms of psychophysical supervenience can, when combined with further assumptions, entail physicalism. I conclude by bringing out some implications that the relationship between supervenience and the Assumptions can have for the use of supervenience in the philosophy of mind.
\end{abstract}




\section{ACKNOWLEDGEMENTS}

My first thanks are really due to Tim Crane for his excellent supervision. I'd then like to thank, for discussion and/or feedback/advice, Lucie Antoniol, Helen Beebee, Chris Daly, John Divers, Michèle Friend, Wallace Fryer, Bob Hale, Frank Jackson, Jonathan Lowe, Lucy O'Brien, John Post, Madhucchanda Sen, Robert Stalnaker, Stephen Yablo.

As for non-professional but still invaluable help, I'd like to thank my parents, Giacomo and Laura, for their constant support and patience. I am, further, grateful to all those friends who did their best to encourage me when I was, in fact, very much in need of being encouraged. 


\section{CONTENTS}

Introduction $\quad 6$

1 Types of supervenience $\quad 12$

$\begin{array}{ll}\text { 1.1 The core idea and the important distinctions } & 12\end{array}$

$\begin{array}{ll}\text { 1.2 Varieties of weak and strong supervenience } & 13\end{array}$

$\begin{array}{ll}\text { 1.3 Fiddling with the distribution-bases } & 22\end{array}$

1.4 'Semi-closure' under identity and quantification, and closure under

$\begin{array}{ll}\text { resplicing } & 32\end{array}$

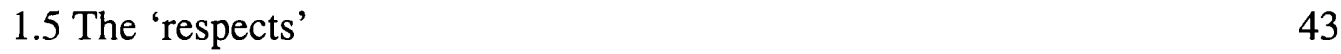

2 Psychophysical Supervenience and the Assumptions 49

$\begin{array}{ll}\text { 2.1 Looking for foundations } & 49\end{array}$

2.2 The Assumptions $\quad 51$

$\begin{array}{ll}2.3 \text { Detectability } & 62\end{array}$

$\begin{array}{ll}2.4 \text { Fundamental properties } & 67\end{array}$

$\begin{array}{ll}2.5 \text { Another attempt: empirical support? } & 74\end{array}$

$\begin{array}{lr}2.6 \text { Causal efficacy } & 80\end{array}$

$\begin{array}{ll}3 \text { Reduction } & 93\end{array}$

$\begin{array}{ll}\text { 3.1 Supervenience as reductive } & 93\end{array}$

$\begin{array}{ll}3.2 \text { Theory reduction and explanation } & 94\end{array}$ 
$\begin{array}{ll}3.3 \text { Ontological reduction } & 102\end{array}$

$\begin{array}{ll}3.4 \text { Implications } & 120\end{array}$

4 Dependence 130

$\begin{array}{lr}\text { 4.1 The Desiderata } & 130\end{array}$

4.2 The less problematic: modal force and the wayward atom 132

4.3 The more problematic: asymmetry and explanation 143

4.4 First attempt to rescue supervenience: versus asymmetry and

$\begin{array}{lr}\text { explanation } & 147\end{array}$

4.5 Second attempt to rescue supervenience: dependence as supervenience 156

$\begin{array}{lr}\text { 4.6 Problematic requirements and physicalism } & 159\end{array}$

$\begin{array}{ll}\text { Conclusion } & 168\end{array}$

$\begin{array}{lr}\text { References } & 178\end{array}$

$\begin{array}{ll}\text { Abbreviations and Definitions } & 190\end{array}$ 


\title{
Introduction
}

Among the most quoted passages in the philosophy of mind, a place of honour is certainly due to the following:

\begin{abstract}
Although the position I describe denies that there are psychophysical laws, it is consistent with the view that mental characteristics are in some sense dependent, or supervenient, on physical characteristics. Such supervenience might be taken to mean that there cannot be two events alike in all physical respects but differing in some mental respect, or that an object cannot alter in some mental respect without altering in some physical respect. ${ }^{1}$
\end{abstract}

Davidson thus drew everybody's attention to the possibility of making use in the philosophy of mind of the concept of supervenience, a concept which had been introduced and used in moral philosophy by writers such as Moore and Hare. $^{2}$ In what follows, I'll first provide a brief sketch of what have been the various stands towards this possibility. Then, I'll put forward what I want to argue by reference to this sketch.

In the seventies and for most of the eighties there was a fair amount of enthusiasm for the possibility of there being an interesting role to be played by supervenience in the philosophy of mind. The main hope was that - as it is suggested by the Davidson passage - psychophysical supervenience could capture and provide a perspicuous formulation of the idea that the mental depends on the physical, an idea regarded as at least one of the core notions of a

\footnotetext{
${ }^{1}$ Davidson (1970a), p. 214.

${ }^{2}$ See Moore (1922), p. 261; and Hare (1952), p. 145.
} 
physicalist position. ${ }^{3}$ (Hence, psychophysical supervenience could, by capturing psychophysical dependence, capture and throw light on at least one of the core notions of physicalism.) Psychophysical reduction, on the other hand, was not supposed to be entailed by psychophysical supervenience. And that was welcome, since it was believed that the yielding of reduction by supervenience would have made psychophysical supervenience implausible (thus causing problems for the physicalist, given the association of psychophysical supervenience with physicalism). ${ }^{4}$

This kind of enthusiasm for psychophysical supervenience did not, of course, reign without, at the same time, some writers expressing reservations. Already in Horgan (1984), for example, one can find the idea that '[supervenience] alone does not explicate materialism as adequately as it initially seems to. ${ }^{5}$ On the whole, however, I think it is fair to say that the vast majority was quite optimistic about the prospect of there being an interesting role to be played by supervenience in the philosophy of mind.

But by the end of the eighties, the tide began to turn. Doubts were cast on the possibility of using psychophysical supervenience alone to capture

${ }^{3}$ See, e.g., Kim (1978; 1979; 1982); Petrie (1987); Post (1987).

${ }^{4}$ See, e.g., Haugeland (1984); Hellman (1985); Petrie (1987); Post (1987). Note, though, the absence of Kim among the ones making the claim about reduction. And note, also, that I am not considering here all the suggested uses of psychophysical supervenience in the philosophy of mind. (I am, for instance, ignoring the appeal to psychophysical supervenience in the debate on mental causation - although something will be said about that, in chapter 2 , when considering the grounds for psychophysical supervenience.) This is because - as it is going to emerge shortly my main interest is focused on the possibility of using psychophysical supervenience to capture positions with respect to the Mind-Body problem.

${ }^{5}$ Horgan (1984), p. 24. 
psychophysical dependence, and, consequently, also on the possibility of using psychophysical supervenience to capture at least one of the core ideas of physicalism. ${ }^{6}$ Indeed, that led to the more general complaint that claims of supervenience fail to be significant as regards to the Mind-Body problem. ${ }^{7}$

In recent years, however, the idea of there being an interesting use to be made of supervenience in the philosophy of mind has won back some sympathy at least in certain philosophical quarters. Writers such as Chalmers and Jackson have revived the idea of using supervenience to capture physicalism. ${ }^{8}$ And we can find the suggestion, in Stalnaker (1996), that supervenience can help to get clearer about kinds of disagreement in the debate on the Mind-Body problem. ${ }^{9}$

All in all we can say that today there are both 'Sceptics' and 'Enthusiasts'. And then, of course, there is always the possibility of arguing for a position which lies somehow in the middle.

This is, indeed, what I am going to do. For the general idea which I would like to defend is that, contrary to what is believed by the Sceptics, a supervenience claim can be quite strong - i.e. it can be quite informative by, e.g., entailing or ruling out many further claims. To this extent, claims of psychophysical supervenience can be used to capture possible positions with respect to the

\footnotetext{
${ }^{6}$ See, e.g., Grimes (1988); Kim (1990a); Charles (1992); Von Kutschera (1992).

${ }^{7}$ See, e.g., Kim (1993; 1997a; 1998); Melnyk (1997); Heil (1998).

${ }^{8}$ See Chalmers (1996) and Jackson (1998). Note that, this time, the idea of using supervenience to capture physicalism is not accompanied by the claim that supervenience is non-reductive. This is because according to these writers physicalism is actually a reductive thesis - although the notion of 'reduction' they have in mind is probably different from the one the early enthusiasts about supervenience were so keen on disassociating from supervenience. For an earlier sample of this kind of position, see Lewis (1983a).

${ }^{9}$ See Stalnaker (1996), pp. 237-8.
} 
Mind-Body problem. On the other hand, though, I also want to concede that the strength of supervenience claims depends, in some cases, on taking stands which are not congenial to all. (Examples of the 'stands' I have in mind are a view of reduction according to which $A$ is reducible to $B$ iff $A$ is derivable from $B$; a view of properties as sets of particulars; a 'weak' view of 'physicality', according to which a property can count as physical as long as it is instantiated exclusively by particulars possessing properties which are used in the physical sciences.) This concession distinguishes my position from that of an Enthusiast.

In chapter 1 I'll be considering the variety of forms of supervenience. The very existence of such a variety can, actually, already be taken as evidence in favour of the expressive power of supervenience claims. For the existence of a variety of forms of supervenience, together with the fact that at least some of these forms capture relations which are inconsistent with each other, means that by making a determinate supervenience claim, one rules out a number of relations in favour of some other. I'll then try to be clear about what are the important distinctions among those which are drawn in terms of differences in forms of supervenience. (And by 'important' I mean both important in the sense that the distinctions in question correspond to substantial distinctions in relations, and important in the sense that the distinctions in question involve features which matter in our context.) In many cases - I'll argue - the important distinctions cut across those which are more commonly made.

In chapter 2 I'll put forward and defend the thesis (Th) that psychophysical supervenience in its full generality can be satisfactorily supported if and only if one is willing to make any of some substantial assumptions (the 'Assumptions') about the nature of mental and physical properties. Given the nature of the 
Assumptions, (Th) - I'll argue - adds potential strength to supervenience claims. Instances of this increase in strength will be considered in chapters 3 and 4 . In chapter 3, I will then argue that psychophysical supervenience can entail reduction under certain understandings of 'reduction', although in some cases it will be possible to reach this conclusion only within certain metaphysical frameworks. I will, further, put to work part of what it is argued in the chapter to show that a claim of psychophysical supervenience can correspond to a position which is inconsistent with some significant positions, such as that of Davidson and that of the Emergentist, in the debate on the Mind-Body problem.

As for chapter 4, what will be considered are going to be the requirements associated with 'dependence' which emerge from the literature on supervenience. (It is by reference to these requirements that relations of supervenience have been claimed, by the Sceptics, to be unsuitable as relations of psychophysical dependence.) I will argue that some of these requirements can be fulfilled on the basis of supervenience alone. Others can't, but then it can be questioned that there are good reasons for thinking that we can't have dependence when these requirements are not satisfied. Finally - I will concede there are requirements which might be problematic for those who want to maintain that psychophysical supervenience can entail psychophysical dependence. These requirements are problematic to the extent that (a) they can't be fulfilled on the basis of supervenience alone; (b) compared to others of the considered requirements there is a stronger case for taking their fulfilment to be necessary for having dependence; (c) there are reasons for remaining unconvinced by attempts at showing that it can't be that their fulfilment is necessary for having dependence. As I will try to show in the last section of the 
chapter, however, the impossibility of fulfilling these requirements on the basis of supervenience alone does not - contrary to what is suggested by the Sceptics - necessarily undermine the possibility of maintaining that we can have physicalism whenever we have at least some determinate forms of psychophysical supervenience. (Still this will be the case, once more, only subject to taking stands which are not congenial to all.)

In the last section of chapter 4 , as in the last section of chapter 3 , an important role will be played by the results of chapter 2 . In the concluding remarks to follow chapter 4, I'll consider some more general implications of these results for the use of supervenience in the philosophy of mind. But, for the moment, let me begin from the beginning, and turn to consider the various types of supervenience. 


\section{Types of supervenience}

\subsection{The core idea and the important distinctions}

As it emerges from the Davidson passage quoted in the Introduction, the core idea of supervenience is that sameness in certain respects (the subvenient respects) yields sameness in certain other respects (the supervenient respects). (When one puts that the other way round, the core idea becomes that differences in the supervenient respects yield differences in the subvenient respects.)

This formulation can be taken as a 'determinable' which can be determined by specifications of the ideas in play. So, for example, the 'respects' can be - as we are going to see in $\S 1.5$ - properties, events, facts, states, predicates, truths, explanations... Moreover, as we are going to see in $\S 1.2$ and in $\S 1.4$, the yielding of sameness in supervenient respects by sameness in subvenient respects can be characterized by different degrees of modal force according to how the notion of this 'yielding' is unpacked. And, further, what is compared when speaking of 'sameness' can be - as we are going to see in $\S 1.3$ - either single individuals, or groups of individuals, or entire possible worlds.

Distinctions in forms of supervenience can be drawn as a result of this variety of determinations. And, accordingly, the notion of supervenience is provided with the potential for expressing important distinctions in relations. (E.g. the distinction between a relation of covariance which holds as a matter of necessity, and one which holds as a matter of contingency; the distinction between a relation of dependence which can, and one which doesn't, involve individuals 
other than the one instantiating the properties which are claimed to be dependent; the distinction between those relations which do, and those which don't, require that the instantiation of a mental property by an individual yield the instantiation, by this same individual, of a physical property which is neither a complement of a physical property, nor the mere conjunction of physical properties.)

This is going to be one of the two general ideas of this chapter. The other is that often, what can be used to express important distinctions in relations are distinctions in forms of supervenience which cut across the ones which are most commonly drawn - or, at least, are different from these latter. Accordingly, what I'll argue to be the important distinctions in forms of supervenience are going to be different from the ones which are most commonly drawn.

\subsection{Varieties of weak and strong supervenience}

Let us begin with the received distinction associated with the following forms of supervenience:

(WS1) A set of properties $A$ weakly supervenes on a set of properties $B$ iff for any world $w$, and for any objects $x$ and $y$, if $x$ and $y$ are $B$-indiscernible in $w$, then they are $A$-indiscernible in $w{ }^{1}$

${ }^{1}$ Cf. Kim (1984a), p. 58. 
$((\forall w)(\forall x)(\forall y)\{(\forall G \in B)[G(x, w) \leftrightarrow G(y, w)] \rightarrow(\forall F \in A)[F(x, w) \leftrightarrow F(y$, w)]\})

(SS1) A set of properties $A$ strongly supervenes on a set of properties $B$ iff for any worlds $w_{j}$ and $w_{k}$, and for any objects $x$ and $y$, if $x$ in $w_{j}$ is $B$-indiscernible from $y$ in $w_{k}$, then $x$ in $w_{j}$ is $A$-indiscernible from $y$ in $w_{k}{ }^{2}$

$\left(\left(\forall w_{j}\right)\left(\forall w_{k}\right)(\forall x)(\forall y)\left\{(\forall G \in B)\left[G\left(x, w_{j}\right) \leftrightarrow G\left(y, w_{k}\right)\right] \rightarrow(\forall F \in A)\left[F\left(x, w_{j}\right)\right.\right.\right.$

$\left.\left.\left.\leftrightarrow F\left(y, w_{k}\right)\right]\right\}\right)$

The WS1/SS1 distinction is meant to express the difference between a relation of covariance which holds as a matter of contingency and a relation of covariance which holds as a matter of necessity. The idea expressed by WS1 is that if $x$ and $y$ are (or were to be) $B$-indiscernible, then they are (or would be) $A$ indiscernible. On the other hand, SS1 is meant to capture the idea that $x$ and $y$ would be $A$-indiscernible also if $y$ were to be $B$-indiscernible from how $x$ is. ${ }^{3}$

Whether this difference is, indeed, best captured in terms of the WS1/SS1 distinction is something which will concern us in $\S 1.4$. For the moment, I want to consider, instead, two other forms of supervenience which are meant to express the difference at issue:

(WS2) A set of properties $A$ weakly supervenes on a set of properties $B$ iff necessarily for any object $x$ and for any property $F$ in $A$, if $x$ has $F$, then there

\footnotetext{
${ }^{2}$ Cf. Kim (1987), p. 81.

${ }^{3}$ For a similar way of expressing the ideas in play here, see Teller (1984a).
} 
exists a property $G$ in $B$ such that $x$ has $G$, and if any $y$ has $G$ it has $F{ }^{4}$

$(\square(\forall x)(\forall F \in A)\{F x \rightarrow(\exists G \in B)[G x \&(\forall y)(G y \rightarrow F y)]\})$

(SS2) A set of properties $A$ strongly supervenes on a set of properties $B$ iff, necessarily, for each $x$ and each property $F$ in $A$, if $x$ has $F$, then there exists a property $G$ in $B$ such that $x$ has $G$, and necessarily if any $y$ has $G$, it has $F .^{5}$

$(\square(\forall x)(\forall F \in A)\{F x \rightarrow(\exists G \in B)[G x \& \square(\forall y)(G y \rightarrow F y)]\})$

WS2 and SS2 are sometimes referred to as 'Modal-Operator Forms of supervenience' (or 'MOFs', for short ${ }^{6}$ ). And they are distinguished from WS1 and SS1, which are referred to as 'Possible-World Forms of supervenience' (or 'PWFs').' Some, furthermore, believe that the MOFs/PWFs distinction is important. ${ }^{8}$ By contrast, I believe that the MOFs/PWFs distinction is not important. What is important is, rather, the distinction between, on the one hand, MOFs where the subvenient sets are not closed under conjunction and complementation, and, on the other hand, PWFs and MOFs where the subvenient sets are closed under conjunction and complementation. ${ }^{9}$ (In what

\footnotetext{
${ }^{4} \operatorname{Kim}(1984 \mathrm{a})$, p. 64.

${ }^{5} \operatorname{Kim}(1984 \mathrm{a})$, p. 65.

${ }^{6}$ Abbreviations and definitions are listed, in alphabetical order, after the References.

${ }^{7}$ Cf., e.g., McLaughlin (1995).

${ }^{8}$ See again McLaughlin (1995).

${ }^{9} \mathrm{~A}$ set is closed under conjunction and complementation iff conjunctions and complements of its members are included among its members.
} 
follows, MOFs where the subvenient sets are closed under conjunction and complementation will be referred to as 'CMOFs', while MOFs where the subvenient sets are not closed under conjunction and complementation will be referred to as 'NCMOFs'.) And this is because it can be shown that CMOFs express the same kind of relation as the one which is expressed by PWFs, whereas the kind of relation which is expressed by NCMOFs is not the same as the one which is expressed by CMOFs/PWFs. But before considering that, I need to say why someone might believe in the importance of the PWFs/MOFs distinction, and why I think, by contrast, that the PWFs/MOFs distinction is not important.

Someone might believe that the PWFs/MOFs distinction is important on the basis of the following reasoning. ${ }^{10}$ There are - it could be argued - distinctions which can be captured by talking in terms of modal operators (by adopting a 'modal-operator talk', an 'MOT') but not by talking in terms of possible worlds (by adopting a 'possible-world talk', a 'PWT'). So, for example, consider the locutions 'logically necessary' and 'analytically necessary'. These locutions are often taken to mean 'logically true' and 'analytically true'. But at least under certain conceptions of analyticity, analytical truths are not the same as logical truths: some analytical truths are not logical truths. (E.g. No bachelor is married - cf. Quine (1953), p. 22-3, where the present distinction between logical and analytical truths is discussed.) The analytically possible worlds ('APWS'), however, are the same as the logically possible worlds ('LPWS'). Therefore PWT can't capture the mentioned distinction between 'analytically necessary'

\footnotetext{
${ }^{10} \mathrm{Cf} . \mathrm{McLaughlin}$ (1995), p. 26-7.
} 
and 'logically necessary'.

If that were true, it would follow that the PWFs/MOFs distinction is important - if not in general, at least in our context. For MOFs would differ in their expressive power from PWFs. And, given our general concern with the expressive power of supervenience claims, this is important.

Still one can resist the claim that the mentioned distinction between 'analytically necessary' and 'logically necessary' can't be captured in terms of PWT. For it does seem possible to conceive of APWS as a proper subset of LPWS. ${ }^{11}$ And such a conception is suitable to capture the kind of distinction between 'logically necessary' and 'analytically necessary' which has been mentioned above. Indeed, if one believes that there are some analytical truths which are not logical truths, then one should, if consistent, believe also that APWS are not the same as LPWS. Think of possible worlds as sets of propositions. Then to say that some analytical truths are not logical truths is to say that there are LPWS which are not APWS insofar as they don't have as members propositions such as the one corresponding to the sentence 'No bachelor is married'. The kind of position above, of someone who believes that some analytical truths are not logical truths while, at the same time, believing that APWS are the same as LPWS, is thus - it appears - actually inconsistent. ${ }^{12}$

\footnotetext{
${ }^{11}$ Cf., e.g., Field (1989).

12 One could object that there is some inconsistency here only because one is conceiving of possible worlds as sets of propositions. Then, however, I'd like to be shown how, by adopting an alternative conception, one can escape the inconsistency. (The general problem here seems to be that any conception of logically/analytically possible worlds is, somehow, going to be related to the notions of logical and analytical truths, so that whatever is claimed about logical/analytical
} 
If it is not true that MOT can capture distinctions which can't be captured by PWT, it follows that we haven't been given any reason for thinking that MOFs differ in their expressive power from PWFs. Accordingly, no good reason has been given either for taking the MOFs/PWFs distinction to be important. In order to find an important distinction in the present context, we'd better turn to something else.

Earlier on I claimed that there is an important distinction between, on the one hand, NCMOFs (i.e. MOFs where the subvenient sets are not closed under conjunction and complementation) and, on the other hand, PWFs and CMOFs (i.e. MOFs where the subvenient sets are closed under conjunction and complementation). And I further claimed that this is so insofar as CMOFs express the same kind of relation as the one which is expressed by PWFs, whereas the kind of relation which is expressed by NCMOFs is not the same as the one which is expressed by CMOFs/PWFs. I now want to say a few words about the equivalence at issue, beginning from what can support the equivalence claim. I'll take the proofs which can be found in the literature to work as proofs of the equivalence between PWFs and CMOFs, but I'll argue that they don't work as proofs of the equivalence between PWFs and MOFs in general.

In Kim (1984a) a proof is given for the claim that WS1 is equivalent to WS2:

[...] First we show that [WS1] entails [WS2]. Assume that for some $\mathrm{F}$ in A, $\mathrm{x}$ has $\mathrm{F}$. We need to show, for some $\mathrm{G}$ in $\mathrm{B}$, that $\mathrm{x}$ has $\mathrm{G}$, and that anything $\mathrm{y}$ with $\mathrm{G}$ has $\mathrm{F}$. Let $\mathrm{G}$

truths can't help having repercussions on what is to be claimed about logically/analytically possible worlds.) 
be the B-maximal property of $\mathrm{x}$ (in any given world under consideration). ${ }^{[13]}$ Then trivially $\mathrm{x}$ has G. To show that anything $\mathrm{y}$ with $\mathrm{G}$ has F: suppose some $\mathrm{y}$ has G. Since both $\mathrm{x}$ and $y$ have $\mathrm{G}$ and $\mathrm{G}$ is a B-maximal property, $\mathrm{x}$ and $\mathrm{y}$ share all properties in B. So by [WS1], $\mathrm{x}$ and $\mathrm{y}$ must share all properties in $\mathrm{A}$. But $\mathrm{F}$ is an $\mathrm{A}$ and $\mathrm{x}$ has $\mathrm{F}$. So $\mathrm{y}$, too, must have $\mathrm{F}$.

Second, to show that [WS2] entails [WS1]: assume $\mathrm{x}$ and $\mathrm{y}$ share all properties in B, and suppose they do not share all properties in $\mathrm{A}$ - that is, for some $\mathrm{F}$ in $\mathrm{A}, \mathrm{x}$ has $\mathrm{F}$ but $\mathrm{y}$ does not. Since $\mathrm{x}$ has F, [WS2] entails that for some $\mathrm{G}$ in $\mathrm{B}$, $\mathrm{x}$ has $\mathrm{G}$, and anything with $\mathrm{G}$ has $\mathrm{F}$. By assumption, $\mathrm{x}$ and $\mathrm{y}$ share all properties in $\mathrm{B}$; so $\mathrm{y}$, too, has $\mathrm{G}$, whence $\mathrm{y}$ has $\mathrm{F}$, yielding a contradiction. ${ }^{14}$

Similarly, in Kim (1987) a proof is given for the claim that SS1 is equivalent to SS2:

To show, first, that [SS1] entails [SS2]: Assume, for any property $\mathrm{F}$ in $\mathrm{A}, \mathrm{x}$ has $\mathrm{F}$ at

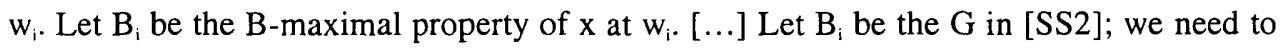
show that necessarily if any $y$ has it, it has F. Suppose otherwise - that is, at some $w_{j}$ there is a $y$ such that $y$ has $B_{i}$ but not $F$. Thus, $x$ has $B_{i}$ at $w_{i}$ and $y$ has $B_{i}$ at $w_{j}$; that is, $x$ and $y$ have the same B-properties in these worlds respectively, and by [SS1], they must have the same A-properties in the respective worlds. Since $x$ has $F$ in $w_{i}, y$ must have $F$ in $w_{j}$, contradicting the supposition. Hence [SS2 holds].

To show the converse: Assume $x$ at $w_{i}$ and $y$ at $w_{j}$ share the same B-properties. Let $F$ be any A-property that $\mathrm{x}$ has at $\mathrm{w}_{\mathrm{i}}$; we need to show that y has $\mathrm{F}$ at $\mathrm{w}_{\mathrm{j}}$. By [SS2] there is a

13 'B-maximal properties' are '[...] the strongest consistent properties constructible in B'. (Kim (1984a), p. 58.)

${ }^{14} \operatorname{Kim}(1984 a)$, p. 64. 
property $\mathrm{G}$ in $\mathrm{B}$ such that $\mathrm{x}$ has $\mathrm{G}$ at $\mathrm{w}_{\mathrm{i}}$, and necessarily (that is, at every world) anything with $\mathrm{G}$ has $\mathrm{F}$. Ex hypothesi, $y$ at $\mathrm{w}_{\mathrm{j}}$ has all the B-properties $\mathrm{x}$ has at $\mathrm{w}_{\mathrm{i}}$. So $y$ has $\mathrm{G}$ at $\mathrm{w}_{\mathrm{j}}$, from which it follows that $\mathrm{y}$ has $\mathrm{F}$ at $\mathrm{w}_{\mathrm{j}}$. Hence, [SS1 holds]. ${ }^{15}$

These equivalence claims can be put in terms of mutual entailments between WS1 and WS2, and between SS1 and SS2. Given our definitions of 'PWFs' and 'MOFs' ('PWFs' = WS1 \& SS1; 'MOFs' = WS2 \& SS2), we can say that Kim thus wants to prove the mutual entailment between PWFs and MOFs.

As far as the entailment of MOFs by PWFs is concerned, what needs to be proved is the claim (C) that it is not possible to have PWFs without MOFs, or, in other words, that PWFs without MOFs leads to a contradiction. This can't be proved if it is possible to find cases which are consistent with some PWF, and inconsistent with some MOF. And if we are dealing with NCMOFs, then it is possible - as it is soon going to be clear - to find cases which are consistent with some PWF, and inconsistent with some MOF. What can be proved, on the basis of Kim's proofs, is thus the equivalence between PWFs and CMOFs, and not the equivalence between PWFs and MOFs in general.

The kind of case which counterexemplifies (C) can be illustrated as follows. Suppose that we have a set of properties $\Phi$ having as members the properties $P$, $P^{\prime}, P^{\prime \prime}$; and a set of properties $\Psi$ having as members the properties $M, M^{\prime}, M^{\prime \prime}$. Then, we'll have a case which counterexemplifies (C) if two individuals $x$ and $y$ are both the same with respect to the $\Psi_{s}$ (given that, say, $M x \& M M^{\prime} x \& M^{\prime \prime} x$, and $\left.M y \& M^{\prime} y \& M^{\prime \prime} y\right)$ and the same with respect to the $\Phi_{S}$ to the extent that $\sim P x \&$

\footnotetext{
${ }^{15} \operatorname{Kim}(1987)$, p. $81-2$.
} 
$\sim P$ ' $x \& \sim P^{\prime}$ ' $x$ and $\sim P y \& \sim P$ ' $y \& \sim P^{\prime}$ ' $y$. For such a case is consistent with a PWF of $\Psi$ on $\Phi$ (we have sameness in both $\Phi_{S}$ and $\Psi S$ ); whereas it is inconsistent with a NCMOF of $\Psi$ on $\Phi$ (both $x$ and $y$ have $\Psi s$ without having any $\Phi_{S}$, unless $\Phi$ is closed under conjunction and complementation; if $\Phi$ is closed under conjunction and complementation, ( $\left.\sim P \& \sim P^{\prime} \& \sim P^{\prime \prime}\right)$ can count as a $\Phi$ property, and $x$ and $y$ can thus count as having $\Phi$ properties).

Another way of putting that is to say that the kind of relation which is expressed by NCMOFs differs from that which is expressed by CMOFs/PWFs to the extent that it requires that any instantiation of a property which belongs to the supervenient set be an instantiation of a property which belongs to the subvenient set and which is neither a complement of a property from the subvenient set, nor the conjunction of properties from the subvenient set. In the psychophysical case, that means that when a psychophysical NCMOF holds, any instantiation of a mental property is an instantiation of a physical property which is neither the complement of a physical property nor the (mere) conjunction of physical properties. (For those who believe that complements of physical properties and mere conjunctions of physical properties are not themselves physical properties, the latter specifications are redundant.) By contrast, PWFs and CMOFs where the supervenient and the subvenient sets are sets having as members, respectively, mental and physical properties, are consistent with the 
existence of (mentally indiscernible) beings such as Cartesian souls. ${ }^{16}$

As we are going to see in chapter 3, this is particularly important when one considers the issue of the relationship between supervenience and reduction. Hence, it will be clear that the distinction between NCMOFs and CMOFs/PWFs is important not only because it corresponds to a substantial distinction in relations, but also because it involves features which matter in our context. For the moment, however, let us turn to some other distinctions.

\subsection{Fiddling with the distribution-bases}

What is compared when speaking of 'sameness', in our determinable formulation of supervenience, can be either single individuals, or groups of individuals, or entire possible worlds. The possibility of comparing groups or worlds, as opposed to single individuals, is important in our context to the extent that - as we are soon going to see - it provides the notion of supervenience with the potential for expressing some features which are frequently thought to characterize the relation between mental and physical properties. The mental properties of an individual are frequently thought to depend not only on physical properties of the individual in question, but also on physical properties of other

\footnotetext{
${ }^{16}$ Note that my way of formulating things here is such as to leave it open whether, given the closure under conjunction and complementation, the subvenient set in question would still be the set of the physical properties, i.e. a set which has only physical properties as members. (That hinges on whether complements and conjunctions of physical properties can count themselves as physical properties.)
} 
individuals. This is the case, for example, for 'broad-content' mental states. Whether supervenience can entail dependence is something which won't actually be considered now, but in chapter 4 . Still, supposing that it can, what is important to note here is that the possibility of taking the indiscernibility claim of a supervenience thesis to be about groups or worlds, as opposed to single individuals, provides the notion of supervenience with the potential for entailing the kind of psychophysical dependence under discussion (henceforth 'broad dependence'), as opposed to a kind of psychophysical dependence where the mental properties of an individual are related exclusively to physical properties of the individual in question (henceforth, 'narrow dependence').

This is commonly put in terms of the distinction between, on the one hand, global and regional forms of supervenience, and, on the other, local forms of supervenience. Global forms of supervenience correspond to the case where what is compared, when speaking of 'sameness' in our determinable formulation of supervenience, are entire possible worlds: a set of properties $A$ globally supervenes on a set of properties $B$ iff worlds which are $B$-indiscernible are also $A$-indiscernible. ${ }^{17}$ Regional supervenience then corresponds to the case where what is compared, when speaking of 'sameness' in our determinable formulation of supervenience, are groups of individuals: a set of properties $A$ regionally supervenes on a set of properties $B$ iff world-regions (i.e. portions of worlds corresponding to subsets of the sets of the inhabitants of the various possible worlds) which are $B$-indiscernible are also $A$-indiscernible. ${ }^{18}$ As for local forms of supervenience, the corresponding case, when speaking of 'sameness' in our

\footnotetext{
${ }^{17}$ Cf. Kim (1984a), p. 68.

${ }^{18}$ Cf. Horgan (1982), p. 37.
} 
determinable formulation of supervenience, is that where what is compared are single individuals: a set of properties $A$ locally supervenes on a set of properties $B$ iff individuals who are $B$-indiscernible are also $A$-indiscernible. ${ }^{19}$

In the next section I'll address the question of whether broad dependence can be entailed only by global and regional forms of supervenience. What I want to do now, by contrast, is to make clear that broad dependence can, indeed, be entailed by global and regional forms of supervenience (under the assumption that supervenience can entail dependence). This can be done by considering the notion of 'indiscernibility' in play in the formulations above. ${ }^{20}$

In the formulation of global supervenience above (henceforth 'GS'), what it is meant by indiscernibility in a certain kind of property (e.g. the $A$ or the $B$ properties) is not that two worlds are the same with respect to the kind of properties in question in that whenever one of the two worlds possesses one of the properties in question, so does the other, and vice versa. ${ }^{21}$ Rather, what it is meant is that two worlds are the same with respect to the kind of properties in question in the sense that the distributions of the properties in question over their inhabitants are the same. A (complete) distribution of properties of a certain kind over a determinate domain (the 'distribution-base') is a (complete) specification, for each individual in the domain and each property of the kind in question, of whether or not the individual has the property. ${ }^{22}$ The notion of world

\footnotetext{
${ }^{19}$ Cf., e.g., Chalmers (1996), p. 33-4.

${ }^{20}$ For simplicity, I will actually focus on the global case. But what I am going to say can easily be seen to be applicable also to the regional one.

${ }^{21}$ Cf. Paull \& Sider (1992), p. 834.

${ }^{22}$ Cf. Kim (1988), p. 113.
} 
indiscernibility under discussion can then be explained as follows. A one-one mapping is established between the inhabitants of the two worlds. The two worlds are then indiscernible with respect to a set of properties $A$ iff the assignments of $A$ properties to individuals related by the mapping are the same.

Given this explication of the notion of 'world-indiscernibility', we can now see how global supervenience can entail broad dependence. The working assumption we need is that the covariance of the $A s$ with the $B s$ (i.e. the yielding of differences in $B s$ by differences in $A s$ ) gives us the dependence of the $A s$ on the $B s$, which is another way of saying that $A / B$ dependence is entailed by $A / B$ supervenience. GS can then entail the dependence of the instantiations of a property $A$ by an individual $x$ on instantiations of $B$ properties by individuals other than $x$. For a failure in $A$-indiscernibility which is due to $x$ being different with respect to $A$ when considered in different worlds, can - given the notion of world-indiscernibility above - yield a failure in $B$-indiscernibility which is due to a failure in $B$-indiscernibilities of individuals other than $x$.

Another lesson which can be taken from the notion of 'indiscernibility' in play in GS is then that there is room for drawing further distinctions within the notion of global supervenience itself. The notion of 'property-distribution', for example, can be either 'individual-specific' or 'structure-specific', according to whether sameness in property-distributions requires the mapping to be between counterparts of the same individual. ${ }^{23}$ (E.g.: The distributions $d_{l}=P a \& \sim P b$, and $d_{2}=\sim P a \& P b$ count as the same under a notion of 'property-distribution' which is structure-specific; they do not count as the same under a notion of

${ }^{23}$ Cf. Kim (1988), pp. 115-16. 
'property-distribution' which is individual-specific.) Corresponding to this distinction, we have a distinction between types of supervenience. ${ }^{24}$ Another distinction - which is going to be illustrated below - is then that between, on the one hand, forms of global supervenience which require the yielding of $A$ indiscernibility by $B$-indiscernibility under any mapping (call these 'SGS', for 'strong forms of global supervenience'), and, on the other hand, forms of global supervenience which require only that there be some mapping under which $A$ indiscernibility is yielded by $B$-indiscernibility (call these 'WGS', for 'weak forms of global supervenience'). ${ }^{25}$ This distinction can be argued to have some importance in our context. I'll thus dwell a bit more on it by considering the following schematic example. ${ }^{26}$

Suppose we have two possible worlds $w_{l}$ and $w_{2}$. In $w_{1}$ the individuals $a$ and $b$ both have the physical property $P$. (For concreteness, you can think of $P$ as the property of having one's $C$-fibres firing.) The individual $a$, however, has the mental property $M$; whereas $b$ doesn't. (For concreteness, again, think of $M$ as the property of being in pain.) Similarly, in $w_{2}$, two individuals $c$ and $d$ have $P$; $d$, then, has $M$, whereas $c$ doesn't.

\footnotetext{
${ }^{24}$ See again Kim (1988), pp. $115 \mathrm{ff}$.

${ }^{25}$ Cf. Stalnaker (1996), p. 227. Stalnaker gives the following definition for SGS:
}

[SGS] (Strong Global Supervenience $=$ Stalnaker's global supervenience $)={ }_{\mathrm{df}}$ [A set of properties] $A$ globally supervenes on [a set of properties] $B$ iff any two worlds that are $B$ indiscernible relative to a mapping from the domain of the one onto the domain of the other are also $A$-indiscernible relative to the same mapping.

For him, then, our GS is in fact equivalent to what we have called 'WGS'.

${ }^{26}$ The example follows the same pattern as the one of the example given in Stalnaker (1996), p. 227. 
Such a case is consistent with a psychophysical form of WGS. For under a mapping which relates $a$ to $d$ and $b$ to $c, w_{1}$ and $w_{2}$ are both physically and mentally indiscernible. On the other hand, the case under discussion is not consistent with a psychophysical form of SGS. For under a mapping which relates $a$ to $c$ and $b$ to $d, w_{1}$ and $w_{2}$ are physically indiscernible without being mentally indiscernible. So, we have a difference in types of supervenience here. Moreover, this difference can be argued to be of some importance in our context. For it allows one to express in terms of a distinction between types of supervenience, a difference in positions with respect to the Mind-Body problem which appears to be quite substantial. (Maybe because of its bearing on whether one is upholding psychophysical dependence? - We'll get back to that in chapter 4.) As it emerges from our example, this is the difference between one who does and one who doesn't allow for the possibility (corresponding to worlds such as $w_{1}$ and $w_{2}$ ) of two individuals being physically indiscernible without being also mentally indiscernible. ${ }^{27}$

Another important distinction, which I now want to consider, can, similarly, emerge from reflection on the notion of 'world-indiscernibility'. Given the oneone mapping requirement, two worlds can be indiscernible, in accordance with the notion of 'world-indiscernibility' above, only if they are worlds of same

\footnotetext{
${ }^{27}$ The difference in question can, of course, be expressed also in terms of weak supervenience. There is, however, a difference (another one!) between expressing the position of someone who doesn't allow for the possibility above in terms of weak supervenience and expressing it in terms of SGS. By expressing it in terms of SGS as opposed to weak supervenience, one ascribes to the position under discussion some additional features, such as, indeed, that of ruling out worldindiscernibilities in the physical which are not accompanied by world-indiscernibilities in the mental. (SGS entails weak supervenience, but weak supervenience does not entail SGS.)
} 
cardinality - i.e. they have the same number of inhabitants. That means that GS does not say anything about, for example, a counterfactual situation which differs from the actual only in the number of individuals involved. Whether this is to be regarded as a serious limitation on the expressive power of GS claims is something which I want to leave open. ${ }^{28}$ What I want to consider is, rather, a relation which does say something about counterfactual situations which differ from the actual only in the number of individuals involved. (This relation is not, strictly speaking, a determinate of our determinable supervenience. Given, however, that it is still very much close to its spirit, I think that we can, after all, regard it as some kind of supervenience relation.)

Here is the relation in question, then:

[SBGS] (Similarity-Based Global Supervenience) $=_{\mathrm{df}}$ A set of properties $A$ is related by SBGS to a set of properties $B$ iff any two worlds that are pretty much similar in respect of $B$ are pretty much similar in respect of $A .{ }^{29}$

\footnotetext{
${ }^{28}$ The issue seems to hinge, once again, on whether one wants to use supervenience claims to express dependence, and, if so, on the notion of dependence in play. One is going to be worried by the limitation under dicussion if, for example, one associates dependence with covariance and wants to use claims of psychophysical supervenience to express the dependence of the mental facts (i.e. the instantiations of mental properties) of our world on the physical facts (i.e. the instantiations of physical properties) of our world. For to say that GS doesn't say anything on counterfactual situations which differ from the actual only in the number of individuals involved, is to say that GS is consistent with cases which differ from the actual in the number of individuals involved and in the mental facts, although they are the same in the physical facts. And that means that GS does not yield the covariance of the mental facts of our world with the physical facts of our world.

${ }^{29}$ Cf. Kim (1988), p. 120.
} 
SBGS is clearly different from GS: there are cases which are consistent with SBGS without being consistent with GS; and there are cases which are consistent with GS without being consistent with SGBS. In the psychophysical case in particular, and for example, the case of a huge difference in mental properties which goes together with a tiny difference in physical properties is consistent with GS without being consistent with SBGS. On the other hand, the case of a complete indiscernibility in physical properties which is accompanied by a slight difference in mental properties is consistent with SBGS (since, in such a case, the two worlds are 'pretty much similar' in respect of $A$ ) without being consistent with GS. Accordingly, the GS/SBGS distinction is suitable to capture the distinction between being willing and being unwilling - when taking a stand on the Mind-Body problem - to allow for the cases in question.

The GS/SBGS distinction has emerged from considerations on the distribution-bases in the global case. Similarly, the WGS/SGS distinction involved the domains referred to in formulations of global supervenience. By way of conclusion of this section, I now want to consider a last case of a difference which emerges from 'fiddling with the distribution-bases'.

This is the case of the distinction between, on the one hand, forms of supervenience where we have the same domain for both $A$-distributions and $B$ distributions (call these 'SDS', for 'Single Domain Supervenience'), and, on the other hand, forms of supervenience where, by contrast, it is left open whether we 
have the same domain for both $A$-distributions and $B$-distributions. (These latter forms of supervenience can, following Kim (1988), be called 'forms of supervenience for multiple domains', or 'MDS', for short. ${ }^{30}$ )

The SDS/MDS distinction is important in our context to the extent that it allows us to express the distinction between on the one hand those who believe in psychophysical token identity, and, on the other, those who, by contrast, want to leave it open whether mental tokens are identical to physical tokens. This latter position, in fact, can be expressed by MDS. Given that MDS does not require that the domain over which the supervening properties are distributed be the same as the domain over which the subvening properties are distributed, it is possible, by subscribing to a psychophysical form of MDS, to subscribe to a form of supervenience of the mental on the physical without, at the same time, committing oneself to the identity of mental tokens with physical tokens. In the limiting case, the 'tokens' (i.e. the particulars) over which the mental properties are distributed could even be entities such as Cartesian souls. In less extreme cases, MDS can be used to express the position of somebody who believes that mental properties are properties of some wholes, while physical properties can

\footnotetext{
${ }^{30}$ Cf. Kim (1988), p. 113:

$<A, D_{1}>$ supervenes on $<B, D_{2}>$ iff every complete distribution of $B$ over $D_{2}$ entails a unique complete distribution of $A$ over $D_{1}$.
} 
be properties only of the mereological sums of the parts of these wholes, and it is to be left open whether mereological sums are identical with wholes; or, indeed, that mental properties are properties of some wholes, while the physical properties on which mental properties supervene are, in some cases, properties only of the parts of these wholes. ${ }^{31}$

Once again, then, the notion of supervenience has proved to be quite resourceful as to its expressive power. It has proved to be capable of expressing some quite determinate features of possible positions with respect to the MindBody problem. Accordingly, also some important differences in psychophysical relations have been proved to be expressible in terms of differences in forms of supervenience. In some cases, then, there is a correspondence between the important differences in relations and the distinctions in forms of supervenience which are commonly drawn. What I want to consider in the next section, by contrast, are cases where important differences in relations cut across the distinctions between forms of supervenience which are most commonly drawn.

Here and elsewhere $D_{1}$ and $D_{2}$ are nonempty sets of individuals, and $A$ and $B$ are nonempty sets of properties.

${ }^{31}$ Cf. Post (1987); and see also the discussion of Post in Melnyk (1995). (Melnyk, for one, actually argues against the identity of the mereological sums and wholes in question.) 


\section{4 'Semi-closure' under identity and quantification, and closure under resplicing}

The distinction between global and local forms of supervenience (henceforth, ' $\mathrm{G} / \mathrm{L}$ ') is commonly believed ${ }^{32}$ to capture the distinction between broad and narrow dependence (henceforth, 'B/N'). ${ }^{33}$ (The working assumption is, of course, that supervenience can capture dependence.) It can be argued, however, that global supervenience is in fact equivalent to strong supervenience. ${ }^{34}$ If so, G/L could not capture B/N. For the equivalence between global and strong supervenience would be the equivalence between global supervenience and a local form of supervenience. It would thus no longer be true that broad dependence can be expressed exclusively by global as opposed to local forms.

Still, the claim that global supervenience is in fact equivalent to strong supervenience can be resisted. It is possible to conceive of a case which is consistent with global supervenience without being consistent with strong supervenience. (This kind of case will be called 'the Petrie case', or 'PC' for short, as it was first brought out in Petrie (1987).) In what follows, I want to

\footnotetext{
${ }^{32}$ See, e.g., Petrie (1987); Paull \& Sider (1992); Moser \& Trout (1995).

${ }^{33}$ To remind the reader: By 'broad dependence' it is meant, here, a kind of dependence relating the instantiations of mental properties by an individual to instantiations of physical properties which are not, necessarily, instantiations of physical properties by that same individual. 'Narrow dependence', by contrast, is used to express the idea that the mental properties of an individual depend exclusively on its physical properties. This is how, at the beginnning of the previous section, it was said that the terms in question would have been used.

${ }^{34}$ See Kim (1984), p. 69. Kim, however, later recanted his argument in the light of the counterexample we are going to consider.
} 
consider PC. I'll argue that the moral which can be drawn from PC is, indeed, that global supervenience is not equivalent to strong supervenience. What can be brought out by reflecting on $\mathrm{PC}$, however, is also that global supervenience is equivalent, if not to strong supervenience in general, at least to forms of strong supervenience where the subvenient sets are 'semi-closed' under identity and quantification. (These forms will be referred to as 'SCSS'.) And I am taking a set of properties $A$ to be 'semi-closed' under identity and quantification iff at least one property construed out of $A$-properties by identity and quantification is included among its members. I'll then argue that SCSS can express broad dependence. Narrow dependence, on the other hand, can, arguably, be expressed by forms of strong supervenience whose sets are neither closed nor semi-closed under identity and quantification ('NCSS'). Hence, it is possible to capture B/N in terms of a distinction between forms of supervenience. But this latter is not $\mathrm{G} / \mathrm{L}$. It is, rather, the distinction between, on the one hand the global supervenience/SCSS pair, and, on the other, NCSS. Once more, then, the important distinction between forms of supervenience is not that which is most commonly drawn.

$\mathrm{PC}$ is a case where two individuals $x$ and $y$ are, in the possible world $w_{l}$, indiscernible with respect to a property $P$ of a set $B$ in that they both instantiate $P$. On the other hand, $x$ instantiates a property $M$ of a set $A$, whereas $y$ doesn't. In another possible world $w_{2}$, then, $x$ instantiates $P$, while $y$ doesn't; and neither $x$ nor $y$ instantiates $M$.

Assuming that $P$ and $M$ are the only properties in, respectively, the sets $B$ and $A, \mathrm{PC}$ is consistent with the global supervenience of $A$ on $B$, but inconsistent with the strong supervenience of $A$ on $B$. For $w_{1}$ and $w_{2}$ are not indiscernible 
with respect to the $B$-properties. So, the fact that they are not indiscernible in their $A$-properties either, does not lead to any inconsistency with the global supervenience of the $A s$ on the $B s$. On the other hand, $x$ in $w_{1}$ and $x$ in $w_{2}$ are indiscernible with respect to their $B$-properties. From the fact that they are not indiscernible also with respect to their $A$-properties, it thus follows that $\mathrm{PC}$ is inconsistent with the strong supervenience of the $A s$ on the $B s$. What PC can be taken to show is then that global supervenience is not equivalent to strong supervenience.

However, consider the semi-closed $B$ which includes among its members, and in addition to $P$, a property $P^{\prime}={ }_{d f} P^{\prime} x$ iff $\exists y(x \neq y \& P y) \cdot{ }^{35}$ In $w_{1}, x$ does instantiate $P^{\prime}$ (in virtue of $y$ instantiating $P$ ). But in $w_{2}$ it doesn't. So, given this semi-closure of $B$, it will no longer be true that $x$ in $w_{1}$ and $x$ in $w_{2}$ are $B$ indiscernible (while being different in the As); $\mathrm{PC}$ will no longer be inconsistent with the strong supervenience of the $A s$ on the $B s$ (while being consistent with the global supervenience of the $A s$ on the $B s$ ), and it won't thus be possible to take PC as a counterexample to the equivalence of the global supervenience of the $A s$ on the $B s$ to the strong supervenience of the $A s$ on the $B s$. Moreover, assuming that $\mathrm{PC}$ is the only kind of counterexample which can be given to Kim's argument for equivalence ${ }^{36}$, what that can be taken to show is that global

\footnotetext{
${ }^{35}$ Cf. Kim (1993), p. 169.

${ }^{36}$ See Kim (1984), p. 69. There is, actually, another (which is, indeed, the only other) counterexample I know of. This can be found in Paull \& Sider (1992), and it can be schematized as follows: $A=\{M\} ; B=\{P ; Q\} ; M=$ ur $M x$ iff $P x \& \exists y Q y ; \mathrm{w}_{1}: P a, \sim Q a, M a, \sim P b, Q b, \sim M b ; \mathrm{w}_{2}$ : $P c, \sim Q c, \sim M c, \sim P b, \sim Q b, \sim M b$. As I hope it can be seen, however, this counterexample can be given exactly the same treatment as the one adopted for PC. I have thus ignored it in the main text to avoid repetition.
} 
supervenience is equivalent to SCSS - i.e. to forms of strong supervenience whose sets are semi-closed under identity and quantification.

The relation which is captured by global supervenience/SCSS is one that does not require that a difference in instantiations of supervenient properties by an individual be always accompanied by a difference which is, and is no more than, a difference in instantiations of subvenient properties by that same individual. (This latter difference can, in accordance with global supervenience/SCSS, be a difference also in instantiations of subvenient properties by other individuals.) Global supervenience/CSS is consistent with PC, where the difference that accompanies the difference in $M$ between $x$ in $w_{1}$ and $x$ in $w_{2}$, is a difference in instantiations of $P$ by $y$. Taking covariance to be enough for dependence, this means that psychophysical forms of global supervenience/SCSS can express broad dependence.

On the other hand, psychophysical forms of NCSS can express narrow dependence - assuming, once again, that covariance is enough for dependence. For NCSS expresses a relation which holds only if a difference in instantiations of supervenient properties by an individual is always accompanied by a difference which is, and is no more than, a difference in instantiations of subvening properties by that same individual. (NCSS is inconsistent with PC, where the difference that accompanies the difference in $M$ between $x$ in $w_{1}$ and $x$ in $w_{2}$, is a difference in instantiations of $P$ by $y$.) So, $\mathrm{B} / \mathrm{N}$ can be captured by the distinction between, on the one hand, global supervenience/SCSS, and, on the other, NCSS. This latter distinction is certainly less common than $\mathrm{G} / \mathrm{L}$ (which, given the equivalence between global supervenience and SCSS, can't capture $\mathrm{B} / \mathrm{N}$ ). Once again, we thus have a case of an important distinction in 
relations which is captured by a distinction in forms of supervenience that cuts across a more common distinction in forms of supervenience, supposed to capture the distinction in relations at issue.

This is, still, not to rule out completely the possibility of expressing $\mathrm{B} / \mathrm{N}$ in terms of a distinction between global and strong supervenience. For suppose that one has qualms about the preservation of propertyhood under identity and quantification. One is not happy, that is to say, with taking any entity which can be constructed out of properties by identity and quantification to be itself a property. Qualms about that are likely to arise from a conception of properties as entities fulfilling some determinate role/s (e.g. properties as truth-makers, grounds for objective resemblances, grounds for causal powers ${ }^{37}$ ); the idea is that not all entities constructed out of properties by identity and quantification would, necessarily, be fulfilling the role/s in question. (More on the relevant conception of properties will be said in $\S 3.3$ when discussing disjunctions of properties.) Or again, suppose that one has qualms about the preservation of physicality under identity and quantification. What would be objected to, this time, would be the necessary physicality of any entity which can be construed out of physical properties by identity and quantification. And the motivation behind this objection could be that taking physicality to be preserved under identity and quantification would lead us to having to regard as physical even entities such as Cartesian souls. (Even these latter, in fact, exemplify properties, of form, say, $P x=(\exists y)(x \neq y \& P y)$, which can be construed out of physical properties by identity and quantification.) Now, both in the case of the sceptic

${ }^{37}$ Cf., e.g., Armstrong (1978). 
about the preservation of physicality, and in the case of the sceptic about the preservation of propertyhood, a claim of strong supervenience of the set of the mental properties on the set of the physical properties amounts to an NCSS. For if one is not willing to take either physicality or propertyhood to be preserved under identity and quantification, and if one wants one's subvenient set to be the set of the physical properties, then one can't take one's subvenient set to be eiher closed or semi-closed under identity and quantification. (Even in the case of semi-closure, as opposed to full closure, there can be no guarantee that the construed property/ies in the set will not be among the ones which the sceptic does not want to count as physical properties.) For these sceptics, then, the global supervenience/strong supervenience distinction will, when considering sets of mental and physical properties, amount to the global supervenience/NCSS distinction. And if $\mathrm{B} / \mathrm{N}$ is expressible in terms of the latter, it will thus be expressible also in terms of the former.

Now, however, note also that by saying this, we have not undermined the idea that what is important is the distinction between, on the one hand, global supervenience/SCSS, and, on the other, NCSS. For $\mathrm{B} / \mathrm{N}$ is, in the case considered, expressible in terms of the global supervenience/strong supervenience distinction only because of the identification of strong supervenience with NCSS. Moreover, this identification was made possible as a result of the adoption of some determinate conceptions of properties and physicality. Under different conceptions, allowing for the preservation of propertyhood and physicality under identity and quantification, it is no longer true that a claim of strong supervenience of the set of the mental properties on the set of the physical properties amounts to an NCSS (and, hence, that B/N can 
be expressed by a distinction between global and strong supervenience). If we want to keep neutral on the notions of propertyhood and physicality in play, we'd thus better speak of the distinction between, on the one hand, global supervenience and SCSS, and, on the other, NCSS, to refer to the distinction in forms of supervenience which can express $\mathrm{B} / \mathrm{N}$.

Considering this distinction, anyway, independently of how we choose to refer to it, our claim above remains in place: we have a case of an important distinction in relations which is captured by a distinction in forms of supervenience that cuts across a more common distinction in forms of supervenience, supposed to capture the distinction in relations at issue. I now want to consider another case of this kind.

This is the case, mentioned at the beginning of $\S 1.2$, of the distinction between relations of covariance which hold as a matter of contingency, and relations of covariance which hold as a matter of necessity. (This distinction will be referred to as 'the contingent/necessary distinction', or ' $\mathrm{C} / \mathrm{N}$ ' for short.) As I said when I first mentioned $\mathrm{C} / \mathrm{N}$, the common idea, in this case, is that $\mathrm{C} / \mathrm{N}$ can be captured by the distinction between weak and strong supervenience (henceforth, 'W/S'). However, similarly to what happened with $\mathrm{B} / \mathrm{N}$, it can be argued that, under a determinate principle of closure of the subvenient set, weak supervenience is in fact equivalent to strong supervenience - or, in other words, that weak supervenience is equivalent to forms of strong supervenience where the subvenient sets are closed under the principle in question. Moreover, it can also be argued that necessary covariance can be expressed by forms of strong supervenience whose sets are not closed under the principle in question; whereas contingent covariance can be expressed by forms of strong supervenience whose 
sets are closed under the principle in question, and by weak supervenience. Once again, then, our distinction in relations (i.e. $\mathrm{C} / \mathrm{N}$, in the present case) could not be expressed in terms of the distinction between forms of supervenience (W/S) which is commonly believed to do the job, but, rather, by a distinction between forms of supervenience which is less commonly drawn - i.e., in the present case, the distinction between, on the one hand, the pair of weak supervenience and those forms of strong supervenience whose sets are closed under the principle to be considered shortly, and, on the other hand, those forms of strong supervenience whose sets are not closed under the principle in question.

The relevant principle in the present context is the one introduced in Bacon (1986) under the name of closure under resplicing:

(CR) Where $\varphi_{\mathrm{w}}$ is the extension of [a property] $\varphi$ at world $w$, and $B_{w}=\left\{\varphi_{\mathrm{w}}\right.$ : $\varphi \in B\}, B$ is also to contain any property $\psi$ such that $\psi_{\mathrm{w}} \in B_{w}$ for each world $w^{38}$

What happens under (CR) can be seen by considering the following example from Oddie \& Tichy (1990).

Suppose that in a possible world $w$, the individuals $x$ and $y$ are red. Given the property $P={ }_{d f}$ being red or blue according as Socrates is or is not snubnosed, if Socrates is snubnosed in $w$, then both $x$ and $y$ will have $P$; if, on the contrary, Socrates is not snubnosed in $w$, then neither $x$ nor $y$ will have $P$. The property $P$ thus weakly supervenes on the set $\{$ being red; being blue $\}$. Now suppose,

\footnotetext{
${ }^{38}$ Bacon (1986), p. 165.
} 
though, that $w^{\prime}$ is a possible world where Socrates is not snubnosed and $x$ is red; and let him be snubnosed in $w$. Then, the individual $x$ will have $P$ in $w$ but not in $w^{\prime}$, while being red (and not blue) in both $w$ and $w^{\prime}$. The property $P$ does not strongly supervene on the set $\{$ being red; being blue $\}$.

However, $P$ can be 'respliced' from the properties of being red and of being blue. Hence, if a set which includes as members the properties being red and being blue is closed under resplicing, it will include also the property $P$. And, with respect to the properties of such a set, $x$ in $w$ and $x$ in $w^{\prime}$ are no longer indiscernible. When considering the set in question, the case at hand can, thus, no longer be taken as a case where we have consistency with weak supervenience and inconsistency with strong supervenience. Moreover, given that strong supervenience entails weak supervenience in any case (i.e. regardless of closure principles on the subvenient set), what that shows is that weak supervenience is equivalent to forms of strong supervenience where the subvenient sets are closed under resplicing. (This is so, of course, under the further assumption that the case at hand is the only kind of case which can be brought to bear against the entailment of strong supervenience by weak supervenience.)

As it emerges from the example, these latter forms of strong supervenience (henceforth, 'RSS'), and weak supervenience, express relations of contingent covariance. RSS and weak supervenience are consistent with a failure of covariance (between $P$ and the non-respliced properties of the subvenient set) when what are compared are individuals of different worlds. On the other hand, those forms of strong supervenience whose sets are not closed under resplicing ('NRSS') express relations of necessary covariance. The failure of covariance 
between $P$ and the properties of the set $\{$ being red; being blue $\}$ is inconsistent with NRSS even when what is compared are individuals of different worlds. Accordingly, $\mathrm{C} / \mathrm{N}$ can be expressed by the distinction between, on the one hand, weak supervenience or RSS, and, on the other, NRSS.

Similarly to what happened with $\mathrm{B} / \mathrm{N}$, scepticism about the preservation of propertyhood and/or physicality under some operations, can lead to an equivalence between a common distinction (i.e., in the present case, W/S) and the distinction I am arguing to be important (i.e., in the present case, the distinction between, on the one hand, weak supervenience or RSS, and, on the other, NRSS). In particular, one might, in the present case, have qualms about the preservation of propertyhood under resplicing. One might, that is to say, be unhappy with taking any entity which can be constructed out of properties by resplicing to be itself a property. And such qualms might be due to adopting a conception of properties as trope-bundles. That would, in fact, imply the imposition of a 'likeness requirement' which is to be satisfied by the instances of those entities that can count as properties, and which would not necessarily be satisfied by the instances of entities obtained by resplicing. (Cf. Bacon (1995).) If so, the claim that the set of the mental properties strongly supervenes on the set of the physical properties would be equivalent to a NRSS claim. For if propertyhood is not preserved under resplicing, and if one wants one's subvenient set to be a set of properties, then one needs one's subvenient set not to be closed under resplicing. Accordingly, for such a person, W/S will, when considering sets of properties, amount to the distinction between weak supervenience and NRSS.

Once again, however, this is so only as a result of the adoption of a 
determinate conception of properties. If we want to keep neutral on the conception of properties adopted (to make the tools offered to express important distinctions available to everybody), we'd better express the distinction in relations at issue by way of a distinction between forms of supervenience where it is explicit whether or not we have closure, as opposed to a distinction in forms of supervenience where closure is denied as a result of the adoption of a determinate conception of properties. In the present case, this is to say that if we want to keep neutral on the conception of properties in play, $\mathrm{C} / \mathrm{N}$ should be expressed in terms of the distinction between, on the one hand, weak supervenience or RSS, and, on the other, NRSS, rather than being expressed by the combination of $\mathrm{W} / \mathrm{S}$ and metaphysical assumptions on the nature of properties.

As we are going to see in chapter $3, \mathrm{C} / \mathrm{N}$ is important when considering the issue of the relationship between supervenience and reduction. The distinction between, on the one hand, weak supervenience or RSS, and, on the other, NRSS, is thus important not only because it corresponds to a substantial difference in relations, but also because it involves features which matter in our context. In the next section, I want to consider whether the same can be said also for some other distinctions in forms of supervenience which are not, once again, among the most commonly drawn. 


\subsection{The 'respects'}

One of the determinable notions in our formulation of the core idea of supervenience are the 'respects' in terms of which the comparison in play in the definition takes place. These 'respects' are frequently taken to be properties, but they can also be events, facts ${ }^{39}$, states $^{40}$, predicates ${ }^{41}$, truths ${ }^{42}$, explanations... ${ }^{43}$

Given that what we are dealing with, here, are different entities, the corresponding supervenience claims will be different. However, there are cases where these distinctions do not matter. These are cases where one of these forms of supervenience entails the other, and what we are interested in is something which can be entailed already by the weaker form. (E.g. fact-supervenience entails property-supervenience, and what we are interested in are forms of reduction which can be entailed already by property-supervenience.) What I want to consider in this section are, by contrast, those cases where this kind of relation can not be established between the forms of supervenience in question. For it is in such cases that there can be room for having an important distinction between forms of supervenience. Moreover, given that in the chapters to follow I intend to focus mainly on property-supervenience ('PS'), what I want to consider in this section is, in particular, whether there are cases where a

\footnotetext{
${ }^{39}$ See, e.g., Kim (1993); Mellor (1993); Menuge (1993); Chalmers (1996).

${ }^{40} \mathrm{Kim}(1982)$.

${ }^{41}$ Davidson (1993); Hellman \& Thompson (1975).

${ }^{42}$ Blackburn (1984); Hellman \& Thompson (1975).

${ }^{43}$ The supervenient and subvenient respects will then be entities belonging to determinate families (e.g. the family of the mental properties; the family of the physical properties). And these are what the relata of supervenience relations are meant to be.
} 
difference in our context might be made by switching to forms of supervenience formulated in terms of entities other than properties. In accordance with what has just been said, these would be cases where PS does not entail the other form of supervenience in question. And, indeed, they would also be cases where sameness in properties does not entail sameness in the other kind of entities in question. For if sameness in properties did entail sameness in the other kind of entities in question, then PS would entail the other form of supervenience. (E.g.: Suppose that sameness in properties entails sameness in facts, and that, in particular, sameness in physical properties entails sameness in physical facts, and sameness in mental properties entails sameness in mental facts. Then, if sameness in physical properties entails sameness in mental properties (i.e. if mental properties supervene on physical properties), it would follow that sameness in physical facts entails sameness in mental facts - i.e. mental facts would supervene on physical facts.)

Let us begin with events. And let us suppose that we are conceiving of events as exemplifications of properties by substances at times. ${ }^{44}$ Within this framework, the issue can arise of whether sameness in events does, actually, require sameness in constitutive substances ${ }^{45}$ If so, sameness in properties is not going to be enough to entail sameness in events. Accordingly, a form of eventsupervenience ('ES') would be stronger than a form of PS. (A case where a psychophysical form of ES holds, and where we have physical sameness, will be a case where we have sameness in the individuals instantiating the physical properties.) On the other hand, though, ES could entail less then PS. For, as there

\footnotetext{
${ }^{44}$ Cf. Kim (1973; 1976).

${ }^{45}$ Cf. Lewis (1986a), ch. 23, p. 250.
} 
can be an issue about the need of having sameness in constitutive substances in order to have sameness in events, so can there be an issue as to whether sameness in constitutive properties is required to have sameness in events. ${ }^{46}$ And if sameness in constitutive properties is not required, then it is hard to see how sameness in events could entail sameness in properties. Moreover, there might, actually, be restrictions on what can count as constitutive properties. ${ }^{47}$ If so, what sameness in events could entail would, at best, be sameness in some properties; PS would be entailed by ES only when dealing with properties of a certain kind (i.e. properties which are constitutive properties of events); forms of PS which are entailed by ES would involve only a specific kind of property. In $\S 3.3$ I'll briefly consider an attempt to put that to work in a discussion which is relevant to our main concern. For the moment, however, let me turn, instead, to what happens, in our context, when events are conceived of in ways different from the one mentioned.

Suppose, for instance, that events are conceived of as temporal parts of objects. ${ }^{48}$ Then, the crucial question becomes that of whether the notion of 'sameness in objects' is to be analysed in terms of 'sameness in properties'. For, unless the notion of 'sameness in objects' is to be analysed in terms of 'sameness in properties', it is hard to see how, under the present conception of events, ES could entail PS or vice versa. And if 'sameness in objects' is not to be analysed in terms of 'sameness in properties', what is entailed by ES is going to be potentially different from what is entailed by PS.

\footnotetext{
${ }^{46}$ Cf., again, Lewis (1986a), ch. 23, p. 250-1; and Kim (1976), p. 48.

${ }^{47}$ Cf., e.g., Lewis (1986a), ch. 23; Kim (1973), p. 10; and McLaughlin (1984), p. 73, on Kim.

${ }^{48}$ Cf. Quine (1981; 1985).
} 
When considering a Davidsonian conception of events ${ }^{49}$, then, the first question that needs to be addressed is whether 'sameness in causes and effects' is to be analysed in terms of sameness in predicates. (If so, it will then be necessary to ask whether sameness in properties entails sameness in predicates.) For under a Davidsonian conception of events, 'sameness in events' is to be analysed in terms of 'sameness in causes and effects'. Thus, if 'sameness in causes and effects' can't be analysed in terms of 'sameness in predicates', it is hard to see how ES could be entailed by predicate-supervenience ('PRS'), or, indeed, by PS in virtue of being entailed by PRS. But 'sameness in causes and effects' does seem to be analysable in terms of 'sameness in predicates'. If two events differ in their causes and effects, then they will differ also in the predicates true of them. Derivatively, there will be a difference also in the predicates true of the individuals 'involved' in the events. So, when events are conceived of à la Davidson, the distinction between ES and PRS does not seem to be important.

Turn now to PRS and PS. The notion of 'physical property' is - at least on a common view ${ }^{50}$ - parasitic on that of 'physical predicate'. To this extent, sameness in physical properties will entail sameness in physical predicates. Once more, then, no difference can - it seems - be made by switching from PS to a form of supervenience formulated in terms of entities other than properties.

This seems to be true also when the entities in question are states. For 'being

\footnotetext{
${ }^{49}$ See Davidson (1967; 1969; 1970b; 1971).

${ }^{50}$ This is the view according to which physical properties are conceived of as the properties picked out by the predicates which feature in the laws of physics - or of some broader category of 'physical sciences'. (See, e.g., Smart (1976), and Jackson (1998).)
} 
in a certain state' appears to amount to 'exemplifying a certain property'. (So, sameness in physical or mental properties, for example, entails sameness in physical or mental states.)

In the case of facts, by contrast, our issue of the significance of a shift from properties to other entities depends on what has been said when considering events as property-exemplifications. For the notion of a fact does seem to be equally analysable as the exemplification of a property by a substance at a time. ${ }^{51}$

Similarly, the significance of the shift from property-supervenience to truthsupervenience or explanation-supervenience depends on the significance of the shift from property-supervenience to forms of supervenience formulated in terms of the entities the truths and explanations in question are about. For sameness in these latter entities entails sameness in the corresponding truths and (true) explanations. So that if sameness in properties entails sameness in the entities in question, then it will also entail sameness in the corresponding truths/explanations. And, in such a case, the distinction between, on the one hand, property-supervenience, and, on the other, truth-supervenience or explanation-supervenience, would not - in the light of what has been argued above - be important.

To conclude and sum up, then, it can be said that the cases where it might

${ }^{51}$ See Chalmers (1996), though, for a notion of 'fact' where the individuals instantiating the properties in question do not play an essential role. Accordingly, property-supervenience can given this conception of 'facts' - entail fact-supervenience without facing the kind of problems which, as we have seen, face the yielding of event-supervenience by property-supervenience under a conception of events as exemplifications of properties by substances at times. 
matter whether, in our formulations of supervenience, we are speaking of properties as opposed to other entities, are the cases of events as propertyexemplifications; of events as temporal parts of objects; of facts; and of truths and explanations concerning the entities mentioned. These are the cases which are to be borne in mind when a claim of property-supervenience can't entail some other claim. For when that happens, a shift to one of the forms of supervenience under discussion might - in the light of what has been argued at the beginning of this section - be of some help. If so, we would have a further instance of the idea that part of the potential strength of the notion of supervenience is due to the variety of its determinates. (Supervenience can entail many different claims because it includes, among its determinates, many theses, each of which entails claims. When we want a claim to be entailed, the more determinates we have, the higher will the probability be of finding, among these determinates, a thesis which does entail the claim in question.) What I am now going to argue, in the context of the next chapter, will point towards other sources of strength. 


\section{Psychophysical Supervenience and the Assumptions ${ }^{1}$}

\subsection{Looking for foundations}

The claim that mental properties supervene on physical properties ('MPS') can more frequently be found as an assumption than as a claim to be argued for. ${ }^{2}$ This is what happens, for example, in Dretske (1998) where MPS is assumed in the proposed account of how reasons explain behaviour. Similarly, in Audi (1993), the causal power of mental properties is defended assuming that mental properties supervene on physical properties.

It seems, however, that there is no reason for believing that MPS is not to be taken as any other claim, i.e. as requiring some argument; the question of how, if at all, one can support MPS seems to be perfectly legitimate.

\footnotetext{
${ }^{1}$ After writing this chapter, I've found some encouragement for my claims by reading Heil (1998), where the possible grounds for supervenience are identified with relations which correspond more or less to my Assumptions. Heil then claims that 'the impression that there could be cases of 'pure' supervenience - supervenience holding tout court, and not in virtue of the holding of something like (1)-(5) [i. e. the grounding relations in question] - is, I contend, unfounded. Supervenience concepts are satisfied by a family of ontologically diverse relations. If that is so, then supervenience claims need to be justified by reference to features of the world like those mentioned in (1)-(5).' (Heil (1998), p. 151.) This is - as it will emerge - clearly in tune with the general gist of the present chapter, although the moral Heil draws from that is different from the one I wish to draw. (See my Conclusion.)

${ }^{2}$ As far as I can see the potential distinctions which have been individuated in $\S 1.5$, have no interesting role to play in connection with what is argued in this chapter. They'll thus be ignored; the discussion will be carried out by considering only MPS.
} 
In this chapter I want to put forward and defend a pretty strong claim as an answer to this question. My claim (call it '(Th)', for Thesis) is that MPS in its full generality (i.e. without any restriction on the mental differences which are meant to entail physical differences) can be satisfactorily supported if and only if one is willing to make any of some substantial assumptions about the nature of mental and physical properties. (Such assumptions will be called 'the Assumptions'.)

(Th) is strong to the extent that its truth requires the inadequacy of any suggestion of support for MPS which does not require one of the Assumptions. Although I can't be sure that what follows is going to be exhaustive, I hope at least to convince the reader of the inadequacy of the suggestions which I'll be considering in $\S 2.3-2.6$. In $\S 2.2$, on the other hand, it is the derivability of MPS from the Assumptions which should appear plausible as a result of what will be argued.

If $(\mathrm{Th})$ is true, it follows that any claim of psychophysical supervenience which can be satisfactorily supported (any 'SSS claim', i.e. any claim of 'Supervenience as Satisfactorily Supported') will, in fact, be a disjunctive claim having the Assumptions as disjuncts. ${ }^{3}$ This implication of (Th) adds potential

\footnotetext{
${ }^{3}$ Not all the Assumptions will, of course, feature as disjuncts in case the upholder of the supervenience claim has reasons for being inclined towards some or one of the Assumptions as opposed to the others. In the limiting case of a preference given to just one Assumption as opposed to the others, the claim would no longer be disjunctive. Shifting from the disjunctive to the non-disjunctive case, however, does not undermine the fact - to be considered shortly - that if $(\mathrm{Th})$ is true, a supervenience claim is made potentially stronger than it would be if (Th) were false. On the contrary, a supervenience claim is in the non-disjunctive case even stronger than in the disjunctive case - as it will be clear from what follows.
} 
strength to supervenience claims. For as long as a supervenience claim is an SSS claim, it will - as we are going to see -mean both a commitment to one or other of some relations which are stronger than supervenience itself, and a commitment to whatever is entailed by any of the Assumptions, which is more than what could be yielded by supervenience if $(\mathrm{Th})$ were false. Instances of this increase in strength will be considered both in chapter 3 and in chapter 4 . For the moment, though, let me turn to the Assumptions themselves.

\subsection{The Assumptions}

Let me begin by listing the Assumptions:

(A1) 'Parts and Wholes':

Necessarily, for every mental property $M, M$ is the property of some whole, and every property of every part of such whole is a physical property.

(A2) 'Identity':

First version $=$ Necessarily, for every mental property $M$ there is a physical property $P$ such that $M=P$ 
Second version $=$ Necessarily, for every mental property $M$ there is a physical property $P$ such that necessarily $M=P$

\section{(A3) 'Higher Order/Lower Order':}

Mental properties are picked out by 'higher-order' predicates, and physical properties are picked out by predicates which are 'lower-order' with respect to the predicates picking out the mental properties. A 'higher-order' predicate is a predicate which picks out a property whose instantiations are entailed by the instantiations of properties picked out by the predicates which are 'lower-order' with respect to the 'higher-order' predicate in question because of the mentioned entailment.

That MPS is entailed by $A l$ can be argued as follows:

(i) If two objects have the same properties, then they are qualitatively identical.

(ii) By (i), necessarily, if we have sameness in properties of parts of wholes (i.e. if we have two wholes which are the same with respect to the properties of their parts), then we have sameness in parts (i.e. we have a one-one mapping 
between parts of the two wholes, and each part is qualitatively identical to the part with which it is related by the mapping).

(iii) Necessarily, if we have sameness in parts (and if each part is not only qualitatively identical to the part with which it is related by the mapping, but it also shares with it the type of relations it bears to the other parts of the whole to which it belongs), then we have sameness in mereological sums - i.e. we have two mereological sums which are qualitatively identical. (The holding of the second antecedent will be expressed by saying that we have sameness in 'partrelations'.)

(iv) A whole is nothing over and above the mereological sum of its parts.

(v) By (iii) \& (iv), necessarily, if we have sameness in parts, and sameness in part-relations, then we have sameness in wholes (i.e. we have two wholes which are qualitatively identical).

(vi) If two things are qualitatively identical, they have the same (intrinsic) properties. 
(vii) By (v) \& (vi), necessarily, if we have sameness in parts, and sameness in part-relations, we have two wholes which have the same (intrinsic) properties.

(Conclusion 1) By (ii), (iii), (vii), and transitivity, necessarily, if we have two wholes which are the same with respect to the properties of their parts, and the same also with respect to part-relations, then we have two wholes which have the same (intrinsic) properties. In other words, the (intrinsic) properties of wholes supervene on the properties and part-relations of their parts.

(A1) Necessarily, for every mental property $M, M$ is the property of some whole, and every property of every part of such whole is a physical property.

(Conclusion 2) From Conclusion 1 and $A 1$ it follows that mental properties supervene on physical properties. ${ }^{4}$

Most of the premisses above are - I take it - uncontroversial. The exception is premiss (iv). Accordingly, the derivability of MPS from $A 1$ is not, admittedly, completely uncontroversial, given that it is controversial that a whole is nothing over and above the mereological sum of its parts.

\footnotetext{
${ }^{4}$ Some of these latter can be relational.
} 
In the case of $A 2$, by contrast, the derivability of MPS (and, in particular, of a RSS form from the first version of $A 2$, and of an NRSS form from the second version of $A 2)$ is completely uncontroversial:

Suppose, first, that the first version holds with the psychophysical identity holding in a world $\mathrm{w}_{1}$. And suppose that $\sim$ RSS $\left(\right.$ for $\mathrm{w}_{1}$ ). For that to be possible, we would have to have two individuals in $\mathrm{w}_{1}$ who are the same with respect to their physical properties without being the same with respect to their mental properties. For them to be different in their mental properties, one of the individuals would have to possess at least one mental property that the other one doesn't possess. But if, necessarily, for every mental property $M$ there is a physical property $P$ such that (in $\mathrm{w}_{1}$ ) $M=P$, then one of the individuals would possess a physical property that the other one doesn't possess. So, the two individuals would not, after all, be the same with respect to their physical properties; the first version of $A 2$ mentioned above can't hold without RSS (for $\mathrm{w}_{1}$ ); the first version of $A 2$ above entails RSS (for $\mathrm{w}_{1}$ ).

Similarly, if we assume that the second version of $A 2$ holds, and that NRSS, we can derive a contradiction imposing the conclusion that the second version of $A 2$ entails NRSS. In order to have $\sim$ NRSS, it would have to be possible to have two individuals, in different worlds, who are the same with respect to their physical properties without being the same with respect to their mental properties. For them to be different in their mental properties, one of the 
individuals would have to possess at least one mental property that the other one doesn't possess. But if, necessarily, for every mental property $M$ there is a physical property $P$ such that, necessarily, $M=P$, then one of the individuals would possess a physical property that the other one doesn't possess. So, the two individuals would not be the same with respect to their physical properties, as it was required to have $\sim$ NRSS; we can't have the second version of $A 2$ without NRSS; the second version of $A 2$ entails NRSS.

Let me now turn to $A 3$. As an example of a higher-order characterization and its corresponding lower-order predicates, take the predicate being coloured and the predicates being blue, being green, being red, etc. Or, again, take the predicate being characterized by the input/output dispositions (i.e. by tendencies to ensue in certain outputs when prompted by certain inputs) associated with pain, and the predicates being in neural state N1, being in neural state $N 2$, being in neural state $N 3$, etc., under the assumption that one is characterized by the input/output dispositions associated with pain if and only if one is either in neural state $\mathrm{N} 1$, or in neural state $\mathrm{N} 2$, or in neural state N3. In both of these higher-order/lower-order cases, the relation in play entails supervenience. (Differences in colour entail differences in being blue, in being green, in being red...; differences in whether or not one is characterized by the input/output dispositions associated with pain entail differences in the states which entail one's being characterized by the input/output dispositions 
associated with pain.) By taking that to hold in all higher-order/lower-order cases, we can say that $A 3$ entails MPS.

There are, however, differences in the type of MPS which is entailed, as there are differences in higher-order/lower-order cases. As I intend to make reference to differences of this kind in the next sections, it will help to devote the rest of this section to the following illustration of the point that there are differences in types of MPS corresponding to differences in higher-order/lower-order cases.

Consider, first, a form of $A 3$ where the mental is claimed to be definable in terms of input/output dispositions. These dispositions are then taken to be explananda which have their dispositional bases as explanantia, and which are, by being assumed to be physical, subject to the following, further, assumption:

[The Completeness of Physics (CP)] Physics is complete; for any physical phenomenon there exists a complete physical explanation. ${ }^{5}$

From that it follows that mental properties are going to have physical properties as their dispositional bases. (From the fact that mental properties are explananda subject to $\mathrm{CP}$, and hence assumed to be somehow definable in physical terms, it follows, from assuming $\mathrm{CP}$, that their explanantia are physical.) Under the assumption that indiscernibility in dispositional bases entails indiscernibility in dispositions, it will thus be possible to derive indiscernibility in mental properties from indiscernibility in physical properties;

\footnotetext{
${ }^{5}$ This is a general version of the more specific principle - restricted to physical effects and causal explanations - which, in the literature, is frequently referred to by making use of the name that I am borrowing. (Cf., e.g., Papineau (1993), and Crane (1995).)
} 
a form of MPS can be derived from a set of assumptions corresponding to a form of $A 3$.

Among these assumptions features the idea that the dispositions associated with the mental are explananda subject to $\mathrm{CP}$. This means that the dispositions associated with the mental have, in our derivation, been assumed to be physical; they have been assumed to be definable in terms of physical inputs and outputs. If, by contrast, the dispositions associated with the mental are not assumed to be physical, what one can do, to derive from CP the form of MPS under discussion, is to argue that indiscernibility in physical input/output dispositions entails indiscernibility in the dispositions relating the inputs and outputs in terms of which the mental is defined. This line of argument, however, is liable to the kind of difficulties which we shall consider in the next section.

Sticking to our derivation, the form of the supervenience obtained is (S1) $\square$ $(\forall x)(\forall F \in M)\{F x \rightarrow(\exists G \in P)[G x \quad \& \quad \square(\forall y)(G y \rightarrow F y)]\} .^{6} M$ and $P$ stand for the sets of, respectively, mental and physical properties. These sets - it is then assumed - are not closed under complementation. In this way S1 can capture the idea that instantiations of mental properties entail instantiations of physical properties robustly understood (i.e. understood as properties which do not feature any complements of physical properties) if the mental supervenes on the physical in accordance with the type of supervenience which can be obtained from our derivation. (Cf. $\S 1.2$.) The first modal operator in $\mathrm{S} 1$ is then assumed to ascribe physical necessity, and the second modal operator is assumed to ascribe metaphysical necessity. (CP is meant to hold in all the physically

\footnotetext{
${ }^{6}$ This corresponds to one way of unpacking the notion of 'strong supervenience'. (See $\S 1.2$. )
} 
possible worlds; and it is true in all possible worlds that dispositions are entailed by those states which are, a posteriori, identifiable with their dispositional bases. $)^{7}$

This is important when one considers the issue of the relation between MPS and reduction. For - as we are going to see in $\S 3.3$ - it might well be that any non-arbitrary way of understanding the notion of property reduction is to be associated with a requirement of predicate co-extensions holding in all possible worlds. If so, whether or not a determinate form of supervenience, such as S1, is enough to give us property reduction will hinge on the kind of features considered in our remarks on S1.

One might then want to introduce specific names do draw distinctions between versions of $A 3$ on the basis of these crucial features. In the present context, let me thus introduce the term ' $A 3-R / R$ ' to refer to versions of $A 3$ yielding forms of supervenience which share all the features of S1 except this latter's being characterized by one specific type of necessity, rather than another, to be associated with the first modal operator. (I am avoiding a specification of the type of necessity, here, for reasons which will become clear in $\S 2.6$. As for my choice of the term ' $A 3-R / R$ ', the idea is that of suggesting that in the versions of $A 3$ under discussion, mental properties are to be regarded as what, in the literature, is sometimes referred to as 'realizables', having physical properties as the corresponding 'realizers'.)

Now consider, by contrast, a version of $A 3$ where the relation between the

\footnotetext{
${ }^{7}$ I am here taking metaphysical necessities to be propositions which are true in all possible worlds, but knowable only a posteriori. (Cf. the kind of accounts of 'metaphysical necessity' which can be found, e.g., in Chalmers (1996) and Jackson (1998).)
} 
mental and the physical is assumed to share all the features of the relation associated with the terms 'determinables' and 'determinates' as originally used. ${ }^{8}$ Here are a couple of these features:

(a) As it is claimed in Johnson (1921), '[...] the understanding of the meaning of [a determinable] carries with it the notion of [its determinates]' ${ }^{9}$ - i.e. the understanding of the meaning of a determinable entails knowledge of what are to count as its determinates. (In Johnson's example, understanding of the determinable 'less than 4' entails knowledge that ' 3 ', ' 2 ', and ' 1 ' are to count as its determinates.)

(b) For a predication of a determinate to be significant, a certain predication of the corresponding determinable must be true. So, for example, the proposition 'Socrates is red' can be significant only if the proposition 'Socrates is coloured' is true. ${ }^{10}$

Both features suggest that the form of supervenience entailed by this version

\footnotetext{
${ }^{8}$ See Johnson (1921); Prior (1949); and Searle (1959). Also Yablo (1992) has, by now, become a classic. In Yablo (1992), however, the use which is made of the terms 'determinables' and 'determinates' departs from the original in precisely those features which we are going to consider.

9 Johnson (1921), p. 177. The relata in play, here, are, of course, predicates rather than properties. I trust, however, that there shouldn't be any problem in seeing how these claims in terms of predicates can still bear on our claims in terms of properties.

${ }^{10}$ See Prior (1949), p. 20.
} 
of $A 3$ is characterized by conceptual necessity. ${ }^{11}$ What is less clear is whether such a form of supervenience is also characterized by two modal operators. ${ }^{12}$ (On that might hinge, as before, what one is to say about the relation with reduction.) To say that we have conceptual necessity, when dealing with a form of supervenience entailed by the version of $A 3$ under discussion, is thus to characterize the form of supervenience in question either as being different from S1 only in that its two modal operators ascribe conceptual necessity, or as being different from $\mathrm{S} 1$ to the extent that, in its case, there is no first operator, and the second ascribes conceptual necessity (as opposed to metaphysical necessity).

Independently of the number of modal operators, the mere fact that we have conceptual necessity is, anyway, already important enough - when, for instance (and once more), dealing with the issue of reduction - to justify the introduction of a specific term to refer to versions of $A 3$ which entail forms of supervenience characterized (in either of the ways considered above) by conceptual modality. Quite predictably, my candidate for such a term is ' $A 3-D / D$ '.

This concludes our overview of the Assumptions. It is now time to argue against the possibility of MPS being satisfactorily supported without appealing to any of the Assumptions.

\footnotetext{
${ }^{11}$ I am here taking conceptual necessities to be propositions which are true in all possible worlds and knowable a priori.

${ }^{12}$ Is the instantiation of a determinable always meant to require the instantiation of one of its determinates? Consider the case of the determinable being coloured - which has, indeed, traditionally been used as an example. Must something always be of a specific colour in order to be coloured?
} 


\subsection{Detectability}

Consider the following principle:

[Detectability (D)] Things (by which it is meant objects, phenomena, etc.) are detectable only in virtue of physical manifestations (by which it is meant manifestations which are describable by appealing to predicates which are used in the physical sciences).

If that were true, it would follow that for a mental difference to be detectable, it would have to be manifestable in a physical difference. That could already be taken, by itself, as a quick argument for psychophysical supervenience especially if the physical difference in question is contemporaneous with the mental difference. ${ }^{13}$ Alternatively - and especially if the physical difference in question is not contemporaneous with the mental difference, while supervenience is conceived of as requiring contemporaneous covariations - one could argue that (D) ensures that the presence of a mental difference entails a physical difference that, by $\mathrm{CP}$, requires, in turn, the presence of a physical

${ }^{13} \mathrm{Cf}$. the 'argument from epistemology' for the supervenience of at least part of the mental on the physical which can be found in Chalmers (1996), p. 73-4. The argument has the following schematic structure:

(i) If $A$ did not supervene on the physical, if differences in the physical were not entailed by differences in $A$, then we would have serious epistemological problems with regard to A.

(ii) We do not have serious epistemological problems with regard to $A$.

$\therefore$ (iii) A supervenes on the physical.

Premiss (i) seems to me to rely on (or, indeed, to amount to) something like (D). 
difference which is contemporaneous to the mental difference. (E.g.: The mental difference manifests itself in virtue of a difference in bodily movements. Given $\mathrm{CP}$, the difference in bodily movements, which is a physical phenomenon, requires, in turn, to be accounted for in terms of some physical phenomenon, such as a difference in physical states causing the bodily movements. And the difference in these physical states is contemporaneous with the mental difference. $)^{14}$ Either way, psychophysical supervenience would have been argued for without making any of the Assumptions. So, if (D) were true, it would be possible - contrary to (Th) - to support psychophysical supervenience without making any of the Assumptions.

But why should we accept (D)? People who are sympathetic to one version of (D) or another, generally reason on something like the following line. Our sense organs - they say - are suitable to detect only the physical, since they work by physical interaction with the environment. So, unless we are willing to allow for the existence of some further and mysterious equipment, we must conclude that we can detect only the physical; the non-physical can be detected only in virtue of physical manifestations. ${ }^{15}$

This way of arguing, however, seems to be resting on a sweeping generalization of the perceptual model. Unless the perceptual model is generalized to any case of acquisition of knowledge, one who is unwilling to require physicality as a necessary condition for detectability can't be pushed into the uneasy position of having to allow for something like an extra sense organ.

But the generalization of the perceptual model to any case of acquisition of

\footnotetext{
${ }^{14}$ Cf. Papineau (1993; 1995).

${ }^{15}$ Cf. Papineau (1993), p. 19-20.
} 
knowledge is, of course, questionable. Why should it be true that we cannot get to know about things except by something like perception? Indeed, in many cases (take, for one, the case of self-knowledge, or again, that of the recognition of the validity of a proof) the suitability of the perceptual model is actually quite controversial. So, why should we think it generalizable to all cases?

To the generalization of the perceptual model to any case of acquisition of knowledge corresponds a sweeping move from a notion of detectability which has been constructed on the basis of the physical/perceptual cases, to a positing of it as a requirement also in the non-physical cases. Again, there is room for objecting to this kind of move - this time by arguing that, once we acknowledge that our starting point for formulating a notion of 'detectability' is to be constituted by what we do and what we do not regard as detectable, it seems to be wrong to take into account nothing but the physical cases.

Given this context, I think that the existence of non-physical cases which we do regard as detectable (but not in virtue of physical manifestations) is enough to provide reasons to reject (D). And, indeed, it seems that we don't need to look too far from the very case which has been considered in attempting to support psychophysical supervenience, to find a case of, precisely, the kind in question. Consider, in fact, the difference between a piece of behaviour such as that of signing a cheque, and a piece of behaviour such as that of testing whether a pen is working properly; or again, the difference between a piece of behaviour such as that of rehearsing for the premiere, and a piece of behaviour such as that of showing to some friends how the role of Richard III should be played. These differences - it seems - are not expressible in terms of predicates which are used in the physical sciences. Yet, they also appear to be detectable in themselves. 
Moreover, it seems that they can be taken as non-physical manifestations in virtue of which some other non-physical difference (i.e. a difference in mental states) is, in turn, detectable. It is thus not true - contrary to what is affirmed in (D) - that everything which is detectable is so in virtue of its physical manifestations.

One could object to the possibility of taking the behavioural differences in question ('BDs') as manifestations of mental differences. Note, however, that when it comes to justifying the rejection of BDs as manifestations of mental differences, it won't be possible to do that on the basis of a conception of the mental which requires manifestations which are exclusively physical, without thereby going a long way towards committing oneself to one of the Assumptions. ${ }^{16}$ (Recall our case of $A 3$ where it was assumed that the mental is to be analysed in terms of relations between physical inputs and physical outputs.)

Seeking a way of defending (D) which does not commit one to any of the Assumptions, one could then object to the claim that BDs are detectable independently of any physical difference. It might well be true - it can be argued - that mental differences are detectable in virtue of behavioural manifestations. These latter, however, are, in turn, detectable in virtue of some physical

\footnotetext{
${ }^{16}$ Note that the same kind of commitment can be argued to be unavoidable also by someone who allows that there is something which is detectable in virtue of BDs, but then takes (D) as applying, if not to everything, at least to what we want to regard as 'scientific'. (The idea is that whatever is detectable in virtue of BDs would not be 'scientific'. So, if we want the mental to be scientifically respectable, we are barred from regarding as a mental difference this something which is detectable in virtue of BDs.) Such a position, in fact, is likely to end up with having to regard as scientifically respectable only a conception of the mental which is, at least partly, defined in terms of physical outputs. (For the idea that (D) can be taken to apply only to what we want to regard as 'scientific', see Loewer (1995).)
} 
differences such as differences in bodily movements. So, in the end, it is not true that there is something (i.e. mental differences) which is detectable independently of any physical manifestation.

According to such a position, pieces of behaviour of the kind which has been considered would thus supervene on bodily movements. This claim, however, is - as it has been anticipated in the previous section ${ }^{17}$ - far from being uncontroversially acceptable. For one thing, it is not difficult to find cases where two individuals can be said to be performing two different types of action while being indistinguishable with regard to their bodily movements. (Think, again, of the former examples of rehearsing for the premiere and showing to some friends how the role of Richard III should be played, and of signing a cheque, and testing whether a pen is working properly.) There are then considerations of a general nature which can be brought to bear against the supervenience of behaviour on bodily movements. So, for example, one could stress that pieces of relational behaviour such as the pointing towards a person in the garden or the pointing towards Alpha-Centauri, seem to be constitutively related to determinate counterfactuals. ${ }^{18}$ (Something is a pointing towards a certain person in the garden rather than a pointing towards Alpha-Centauri just in case if the person pointed at, but not Alpha-Centauri, had been differently located, then the agent would have moved differently, and if Alpha-Centauri, but not the person pointed at, had been differently located, the agent would not have moved differently.) Differences in this kind of counterfactuals are not reflected

\footnotetext{
${ }^{17}$ The defence of (D) which we are considering here corresponds to one of the two kinds of derivation of MPS from versions of $A 3$ which have been considered in the previous section.

${ }^{18}$ Cf. Peacocke (1993).
} 
in differences in actual bodily movements. The fact that I would have moved in a different way if the person I am pointing to had been located differently, does not seem to depend on how I am moving now. It seems, indeed, possible to say that from the fact that two individuals are, as a matter of fact, now indistinguishable in their bodily movements, it does not follow that they could not have been so. And this allows for the possibility of the two individuals being distinguishable in the kind of counterfactuals in question, while being indistinguishable in their actual bodily movements. So, two individuals can be distinguishable in pieces of relational behaviour, while being indistinguishable in their actual bodily movements. (Note that this can, in our context, be used against (D) insofar as, given the line of thought which - as we have seen generally lies behind (D), the physical manifestations in (D) are required to be actual as opposed to counterfactual.)

It thus definitely seems that (D) can't be satisfactorily defended without making any of the Assumptions. If so, it follows that the strategy considered in this section is unsatisfactory as a way of supporting MPS without making any of the Assumptions. Similarly, but more generally, also the strategy to be considered in the next section will be argued to be unsatisfactory as a way of supporting MPS.

\subsection{Fundamental properties}

A strategy for supporting MPS that is common to some of the arguments considered in the last two sections, is that of breaking the entailment from 
physical indiscernibility to psychological indiscernibility into sub-entailments, by introducing in the derivation a third kind of indiscernibility. So, for example, considering one version of $A 3$ in $\S 2.2$, psychological indiscernibility was derived from indiscernibility in input/output dispositions which, in turn, was derived from indiscernibility in the kind of physical properties with which dispositional bases can be identified. Similarly, in one of the arguments based on (D), psychological indiscernibility at a time $t_{l}$ is derived from physical indiscernibility at a later time $t_{2}$, which, in turn, is derived from physical indiscernibility at $t_{1}$.

The situation can be schematized as follows:

$$
\begin{aligned}
& \mathbf{A}=\text { physical indiscernibility } \\
& \mathbf{B}=\text { intermediary kind of indiscernibility } \\
& \mathbf{C}=\text { psychological indiscernibility } \\
& \mathbf{A} \rightarrow \mathbf{B} \rightarrow \mathbf{C}
\end{aligned}
$$

The idea is that the task of supporting the entailment from $\mathbf{A}$ to $\mathbf{C}$ is made less difficult by breaking it into two easier 'sub-tasks' - that of supporting the entailment from $\mathbf{A}$ to $\mathbf{B}$, and that of supporting the entailment from $\mathbf{B}$ to $\mathbf{C}$. In the scheme, the longer the arrow (the further the distance between the letters), the more difficult is to support the entailment between what they correspond to. And that captures another important phenomenon. This is that the easier the step from what corresponds to $\mathbf{A}$ to what corresponds to $\mathbf{B}$ (or from what corresponds to $\mathbf{B}$ to what corresponds to $\mathbf{C}$ ) is made to be supported in virtue of certain 
assumptions (the shorter the distance from $\mathbf{A}$ to $\mathbf{B}$ - or from $\mathbf{B}$ to $\mathbf{C}$ ), the harder is to support the step from what corresponds to $\mathbf{B}$ to what corresponds to $\mathbf{C}$ (or, in the other case, from what corresponds to $\mathbf{A}$ to what corresponds to $\mathbf{B}$ ); the distance between $\mathbf{B}$ and $\mathbf{C}$ (or, in the other case, from $\mathbf{A}$ to $\mathbf{B}$ ) has increased.

This is something which could - arguably - be noted in the derivation of MPS from the form of $A 3$ which we have considered. For, if the inputs and outputs are defined exclusively in terms of physical properties, there are good prospects for supporting the step from indiscernibility in the kind of physical properties with which dispositional bases can be identified to indiscernibility in input/output dispositions; but the step from indiscernibility in input/output dispositions to psychological indiscernibility might become problematic. If, on the other hand, the inputs and outputs are not defined exclusively in terms of physical properties, it is the step from indiscernibility in the kind of physical properties with which dispositional bases can be identified to indiscernibility in input/output dispositions that becomes problematic; whereas the step from indiscernibility in input/output dispositions to psychological indiscernibility becomes - arguably - easier to support.

Also the strategy that I want to consider in this section is characterized by this kind of phenomenon. Indeed, as we are going to see, it provides instances of limiting cases where the step from $\mathbf{B}$ to $\mathbf{C}$ has almost disappeared, thus making the one from A to B very hard to support. But, before we can see that, we need to know what it is that, in the strategy to be considered, $\mathbf{B}$ is meant to stand for.

The idea - drawn from Lewis (1994a) - is that $\mathbf{B}$ can be taken to stand for 'indiscernibility in fundamental properties'. Lewis, in fact, argues for the supervenience of everything (and, hence, a fortiori, of the Mental) on the 
fundamental properties. The step from $\mathbf{B}$ to $\mathbf{C}$ is then meant to be $a$ priori; it is an a priori principle - Lewis claims - that 'every contingent truth must be made true, somehow, by the pattern of coinstantiation of fundamental properties and relations' ${ }^{19}$

This claim can be made more or less substantial depending on what it is meant by 'fundamental properties'. If 'fundamental properties' cannot be characterized except in terms of their constituting the basis (the 'foundation') from which all the rest follows, then it will indeed follow that 'every contingent truth is made true, somehow, by the pattern of coinstantiation of fundamental properties and relations'. Such a conclusion, though, will be trivially true. Moreover, the step from physical indiscernibility to indiscernibility in fundamental properties will become at least as hard to defend as, indeed, the step from physical indiscernibility to psychological indiscernibility. No progress would have been made. (No wonder: in order to support the step from physical indiscernibility to indiscernibility in fundamental properties, it will have to be argued that, in our world, the fundamental properties coincide with the physical properties. Given the aforementioned characterization of 'fundamental properties', that amounts to claiming that the physical properties are the subvenient properties. The distance from $\mathbf{B}$ to $\mathbf{C}$ has thus collapsed; that from $\mathbf{A}$ to $\mathbf{B}$ has stretched to the extent of constituting all that has to be proved.)

Lewis, however, does characterize the fundamental properties also as the 'perfectly natural properties'. The concept of 'perfectly natural property' is actually quite difficult to pin down. (As Lewis himself points out, there are

\footnotetext{
${ }^{19}$ Lewis (1994a), p. 412.
} 
problems with giving a definition of 'natural properties' if one wants to make the extended use of them that he does, while, at the same time, avoiding circularity cf. Lewis (1986b), p. 63.) On the basis of what Lewis writes in Lewis (1983a) and Lewis (1986b), however, it is possible to individuate at least two features of the perfectly natural properties which might shed some light on the derivation from indiscernibilty in fundamental (= perfectly natural) properties to psychological indiscernibility.

First, perfectly natural properties are intrinsic. (See Lewis (1983a, p. 357; 1986b, p. 61).) Indiscernibility in perfectly natural properties entails indiscernibility in $\left(\mathrm{some}^{20}\right)$ intrinsic properties. From that it follows that the claim that everything supervenes on perfectly natural properties can be put in terms of a supervenience on (a certain set of) intrinsic properties.

But why should one believe that everything (and, hence, a fortiori, the Mental) supervenes on (a specific set of) intrinsic properties? Unless one is given reasons for believing that, no progress can be made by pointing out that perfectly natural properties are intrinsic.

At this point, however, one might wonder whether the claim under discussion is not equivalent to what, elsewhere, Lewis calls 'Humean supervenience'. ${ }^{21}$

${ }^{20}$ Indiscernibility in perfectly natural properties entails indiscernibility in just some intrinsic properties (as opposed to entailing indiscernibility in intrinsic properties in general) insofar as, although all perfectly natural properties are intrinsic, not all intrinsic properties are perfectly natural. (See Lewis (1983a, p. 357; 1986b, p. 61).)

${ }^{21}$ See, e.g., Lewis (1986a), p. ix-x:

Humean supervenience is named in honor of the greater denier of necessary connections. It is the doctrine that all there is to the world is a vast mosaic of local matters of particular fact, just one little thing and then another. [...] We have geometry: a system of external relations of spatio-temporal distance between points. Maybe points of spacetime itself, maybe point-sized bits of matter or aether or 
Against this suggestion one can, admittedly, point out that Humean supervenience is claimed to be an empirical issue. (Cf. Lewis (1986a), p. x \& p. xi.) In contrast - as we have seen - the principle that 'every contingent truth must be made true, somehow, by the pattern of coinstantiation of fundamental [= perfectly natural $=$ intrinsic] properties and relations' is meant to be true $a$ priori. That suggests that by 'Humean supervenience' Lewis must, after all, mean something different from the supervenience of everything on the intrinsic properties with which the perfectly natural properties can be identified.

Suppose, however, that, contrary to what has just been suggested, the supervenience of everything on the relevant intrinsic properties is, after all, Lewis's 'Humean supervenience'. If not Humean supervenience, it could at least be a determinable of Humean supervenience. This latter would be a determinate of the claim under discussion to the extent that '[...] Humean Supervenience is yet another speculative addition to the thesis that truth supervenes on being [...] it says that in a world like ours, the fundamental properties are local qualities: perfectly natural intrinsic properties of points, or of point-sized occupants of points $[\ldots]]^{22}$ If so, the empirical nature of Humean supervenience would be perfectly consistent with the claim under discussion being a priori. What could be taken to be a posteriori would be that the subvenient perfectly natural intrinsic properties are perfectly natural intrinsic properties of points, or of pointsized occupants of points.

fields, maybe both. And at those points we have local qualities: perfectly natural intrinsic properties which need nothing bigger than a point at which to be instantiated. For short: we have an arrangement of qualities. And that is all. There is no difference without difference in the arrangement of qualities. All else supervenes on that.

${ }^{22}$ Lewis (1994b), p. 474. (Italics mine.) 
Would it then follow that we would thus have reasons for believing the claim under discussion? What Lewis does - as he puts it himself ${ }^{23}$ - is to uphold not so much the truth of Humean supervenience as its tenability. That suggests that the arguments he provides are to be read as a defence against objections to the thesis, rather than as some positive support for it. If so, it follows that we would still lack some positive support for the claim that everything (and, hence, $a$ fortiori, the Mental) supervenes on (a specific set of) intrinsic properties.

Maybe things are different with the second feature of the perfectly natural properties which promises to shed some light on the derivation from indiscernibility in fundamental (= perfectly natural) properties to psychological indiscernibility. Perfectly natural properties are used to delineate the notion of 'duplication': two things are duplicates iff they have exactly the same perfectly natural properties. ${ }^{24}$ If indiscernibility is regarded as a case of duplication ${ }^{25}$, it will then follow that, on the basis of the given definition of duplicates, any case of indiscernibility can be regarded as a case of sameness in perfectly natural

${ }^{23}$ Cf. Lewis (1986a), p. ix.

${ }^{24}$ See Lewis (1983a, p. 356; 1986b, p. 61).

${ }^{25}$ Lewis actually distinguishes between duplication and indiscernibility:

Two things are duplicates iff they have the same intrinsic qualitative character; and that is a matter of the perfectly natural (hence ex officio intrinsic) properties of those things and their parts, and of the perfectly natural external relations of their parts. Two things are indiscernible iff they have the same intrinsic and extrinsic qualitative character. [Lewis (1986b), p. 62-3.]

Given, however, that the extrinsic qualitative character of the two things which are indiscernible turns out to be definable in terms of perfectly natural properties and relations (see Lewis (1986b), p. 63), I think that psychological indiscernibility can follow from sameness in perfectly natural properties even when the concept of 'indiscernibility' is taken in Lewis's sense. The only difference with a case where indiscernibility is regarded as a case of duplication (in Lewis's sense) is that we'll have a form of supervenience which is global rather than local. 
properties; a fortiori, psychological indiscernibility will follow from indiscernibility in perfectly natural properties.

The problem here is rather that, in this way, it is, once again, the step from $\mathbf{A}$ to $\mathbf{B}$ that becomes problematic. If the perfectly natural properties are characterized in terms of what ensures indiscernibility, then, if in order to support the step from $\mathbf{A}$ to $\mathbf{B}$ it is necessary to argue for the identification of the perfectly natural properties with the physical properties, it follows that what needs to be proved is that indiscernibility in general can be ensured by indiscernibility in physical properties. And that looks like an even more general form of the very supervenience claim which the whole of the argument was meant to prove.

\subsection{Another attempt: empirical support?}

Consider the following claims: [1] mental differences have always been found to be accompanied by physical differences; [2] by induction, this can be generalized in such a way as to acquire modal force - or, alternatively, modal force can be ascribed to the correlations on the grounds that such ascription has led to success/successful predictions in our scientific practice.

Claim [1] can be taken to hold as long as long as each of the mental differences in question has always been found to be accompanied by some physical difference, yet not necessarily the same in every case. It is possible, however, to make the claim more ambitious by correlating each mental difference with some specific physical difference/s. So, for example, one can 
claim that a difference in whether or not one is in pain has always been found to be accompanied by a difference in whether or not one is in the neural state $\mathrm{N} 1$, or in the neural state $\mathrm{N} 2$, or in the neural state N3. The claim would thus be that what has always been found to hold are conjunctions of the following form:

(A) $M D 1$ (= a determinate mental difference) \& PDI (= a determinate physical difference) ${ }^{26}$

Moreover, these conjunctions can further be claimed to have held in virtue of correlations of the following form:

$\left(A^{\prime}\right)(\forall x)(M x \leftrightarrow P x) .{ }^{27}$

And, in accordance with claim [2], the fact that a conjunction of type $A$ has always been found to hold can be taken to yield, by inductive generalization ${ }^{28}$, a conditional of the following form:

(B) In any possible world $R P W$ belonging to some determinate set $R$, if we have a case of mental difference $M D 1$, we have a case of physical difference PD1.

(A conditional of type $B$ can be derived from repeated instances of a conjunction of type $A$ by inductive generalization insofar as the past set-ups in which the conjunctions have held are regarded as worlds belonging to the set $R$, and a conjunction of type $A$ is taken to support a conditional of the following type:

$\left(A^{\prime \prime}\right)$ If we have the mental difference $M D 1$, then we have the physical

\footnotetext{
${ }^{26}$ Cf., e.g., Conee (1995), p. 277.

${ }^{27}$ Cf. Kim (1979) for a similar claim. $M$ stands for a mental property, while $P$ stands for either a physical property or a disjunction of physical properties.

${ }^{28}$ Cf. Post (1995), p. 75.
} 
difference $P D 1.)^{29}$

Conditionals of type $B$ are forms of psychophysical supervenience. Moreover, they can - as it has just been shown - be supported without making use of any of the Assumptions. Unless one decides - as I won't do - to argue that the kind of support considered above is unsatisfactory, one thus gets very close to having a counterexample to (Th). Still, (Th) is the thesis that MPS in its full generality (i. e. without any restriction on the mental differences which are meant to entail physical differences) cannot be satisfactorily supported without making use of any of the Assumptions. So, in order to counterexemplify (Th), what needs to be shown supportable without making use of any of the Assumptions is something like the following claim:

(C) For any mental difference $M D$, there is, in any possible world $R P W$ belonging to the set $R$, a physical difference $P D$ such that if we have $M D$, then we have $P D$.

One can then argue that $(C)$ can be derived from conditionals of type $B$ by inductive generalization. It can be argued that a number of instances of conditionals involving specific mental and physical differences allows us, by induction, to generalize to all mental differences the holding of the kind of conditional in question. But in our case - I'll now try to show - there are

\footnotetext{
${ }^{29}$ Note that there is a possibility of $M D 1$ and $P D I$ being 'cross-world' - i.e. of the individuals compared being in different worlds - if one allows for comparisons which involve different setups. If so, what would be obtained would actually be something different from $B$. Still, that does not matter for the problems which I am going to raise.
} 
problems with drawing this kind of inductive generalization. ${ }^{30}$

In order for our inductive generalization to go through, we must have no reason to suspect that our evidence is constituted by an unrepresentative crosssection, that our type- $B$ conditionals hold only because of some special features which do not characterize the cases we want to generalize to. But if it is possible to draw a principled distinction between the type- $B$ conditionals we have, and the cases we want to generalize to, then we would indeed have reason to suspect that our evidence is constituted by an unrepresentative cross-section. For, in such a case, we could suspect that our type- $B$ conditionals hold only because of some special features which do not characterize the cases we want to generalize to. ${ }^{31}$ So, if it is possible to draw a principled distinction between the type- $B$ conditionals we have, and the cases we want to generalize to, our generalization becomes problematic.

This seems, indeed, to be the case. For one thing, our evidence, as to type- $B$ conditionals, seems to be restricted to differences involving phenomenal states as opposed to propositional attitudes. (Nobody, as far as I know, has ever maintained that when dealing with propositional attitudes, type- $B$ conditionals can be established on the basis of the kind of evidence considered above - i.e.

\footnotetext{
${ }^{30}$ As the kind of support for MPS which we are interested in is not one which would make MPS trivial, I am not addressing the possibility of deriving $(C)$ by arguing for the constant holding of some $P D$ in any $R P W$.

${ }^{31}$ If we don't suspect that, it is because we choose to disregard the special features, judging them incapable of undermining the possibility of inductive generalization, on the grounds of the belief that what matters are the similarities between evidence and cases we want to generalize to. Then, however, it is hard to see how this could be argued without assuming some (unificatory) analysis of the mental, as in the Assumptions.
} 
correlations of type $\left(A^{\prime}\right)$ and/or conjunctions of type (A.).) If so, it follows, by what has just been argued, that there are going to be problems with deriving $(C)$ from type- $B$ conditionals as soon as one tries to generalize to propositional attitudes. (It will be possible to draw a principled distinction between, on the one hand, our evidence, and, on the other, the kind of cases we want to generalize to: on the one hand we have phenomenal states, on the other we have propositional attitudes.)

Moreover, there are going to be problems with deriving $(C)$ from type- $B$ conditionals also as soon as one tries to generalize to mental differences involving dispositional states which are defined in terms of complex inputs. ${ }^{32}$ For, in the case of such states, it is difficult to have repeated instances of type- $A$ ' correlations, and type- $A$ conjunctions. (The complexity of the inputs makes it easier to have a change in inputs whenever we have a change in set-up. Hence, it is difficult to have instances of the same dispositional state/mental difference in different set-ups. ${ }^{33}$ ) Once more, it is thus possible to draw a principled distinction between our evidence and the cases we want to generalize to. (The crucial contrast here, on the basis of which the distinction is to be drawn, is that of mental differences involving dispositional states defined in terms of complex

\footnotetext{
${ }^{32}$ Note that this problem can - contrary to our first problem - affect also phenomenal states as long as a dispositional analysis of these latter is not excluded.

${ }^{33}$ Example: The mental state $M$ is defined as the disposition to be in the physical state $P$ whenever one is prompted by a group of perceptual stimuli, $P^{\prime}, P^{\prime \prime}, P^{\prime \prime}$. Our evidence for cases of state $M$ will thus be cases where an individual is prompted by the perceptual stimuli $P^{\prime}, P^{\prime \prime}, P^{\prime \prime}$, and it is in the physical state $P$. The more stimuli are required to have evidence for $M$, the easier will it be for a set-up to fail to provide evidence for $M$ as a result of the absence of one or some of the stimuli in question.
} 
inputs versus mental differences involving dispositional states defined in terms of simple inputs.)

Finally, consider the case of mental differences involving many mental states. Here again it is difficult to have repeated instances of type- $A$ conjunctions. (The more (specific) states in which two individuals must differ whenever we want to say that a determinate difference $M D 1$ holds, the more difficult will it be to have many cases where $M D 1$, as opposed to some other mental difference, can be said to hold.) It is thus plausible to maintain that our evidence, for grounding type- $B$ conditionals, is restricted to mental differences which do not involve many mental states. (Especially if the mental states in question are taken to include relational states.) Once more, it is possible to draw a principled distinction between, on the one hand, our evidence, and, on the other, the kind of cases we want to generalize to. (The relevant contrast, here, is that between, on the one hand, mental differences which do not involve many mental states, and, on the other, mental differences which involve many mental states.) If what has been argued is true, this is to be taken as a further problem for deriving $(C)$ in the way considered in this section. And problems in deriving $(C)$ in the way considered in this section mean problems in counterexemplifying (Th) on the kind of grounds considered in this section. Hence, I conclude that (Th) can't be satisfactorily counterexemplified on the kinds of grounds considered in this section. I shall now turn to the next, and last, attempt to provide a satisfactory counterexample. 


\subsection{Causal efficacy}

The line of argument that I would like to consider in this section can be presented as follows: ${ }^{34}$

(i) The Mental is causally efficacious with regard to the Physical - i. e. there are causal transactions with the Physical which take place in virtue of the mental properties of the relata. (Call this 'CEM'.)

(ii) MPS features as part of what is necessary for CEM.

$\therefore$ (iii) MPS holds.

Premiss (ii) is supported by an argument of the following form:

(i) CEM requires $\mathrm{X}$.

(ii) $\mathrm{X}$ requires MPS.

$\therefore$ (iii) CEM requires MPS.

Now consider the following argument to see what ' $\mathrm{X}$ ' stands for.

\footnotetext{
${ }^{34}$ I'll sometimes refer to this line of argument by calling it 'the argument from the causal efficacy of the mental' (or 'ACEM', for short).
} 
(i) CEM (which, remember, means the causal efficacy of the mental with regard to the physical) is incompatible with the conjunction of the following assumptions ${ }^{35}$ :

(H) Homogeneity: 'causal efficacy' means the same when the concept is applied to the mental as when the concept is applied to the physical.

(CCP) The Causal Closure of the Physical: any physical effect can be completely accounted for in terms of the causal efficacy, with regard to it, of certain physical properties.

(CEE) Causal Explanatory Exclusion ${ }^{36}$ : for any effect there is no more than a single complete and independent causal explanation.

(PD) Property Dualism: mental properties cannot be identified with physical properties.

(ii) The only assumption which can reasonably be rejected is $(\mathrm{H})$.

$\therefore$ (iii) CEM requires a rejection of $(\mathrm{H})$.

\footnotetext{
${ }^{35}$ Cf. Crane (1995).

${ }^{36}$ Cf. Kim (1989b; 1990b).
} 
This means that any satisfactory way of accounting for CEM will, given the premisses above, have to be at least compatible with a rejection of $(\mathrm{H})$. Call this kind of account a 'No-(H) Account' - 'NHA' for short. (An NHA will thus be an account of the causal efficacy of the mental which (a) is satisfactory, and (b) is compatible with a rejection of $(\mathrm{H})$.) Then, the crucial claim of ACEM is that any NHA entails MPS. What I want to argue is that any account which stands any chance of being an NHA entails, in addition to MPS, at least one of the Assumptions. ${ }^{37}$ There will be only one exception to this rule. It will be a candidate NHA which is grounded in claims of an empirical nature. These claims will - in accordance with what has been argued in the previous section be maintained to have a chance of being satisfactorily supported only for some mental states. Hence it will be possible to conclude that MPS in its full generality can't be got, via ACEM, without commitment to at least one of the Assumptions; (Th) can't be counterexemplified by means of ACEM.

But let me begin by considering a candidate NHA which does not seem to require a commitment to the Assumptions. According to this candidate NHA, CEM is ensured by the truth of certain conditionals which, in their turn, are ensured by the supervenience of the mental on the physical. The conditionals in question are of the form $\square\left(\sim M c \rightarrow \sim P^{*} e\right)-$ where $M$ stands for a causally efficacious mental property, $P^{*}$ stands for a physical property, and $c$ stands for

\footnotetext{
${ }^{37}$ In a sense, I won't thus take issue with ACEM itself; I'll be granting all the premisses above. For some, that is, of course, already being too generous. (See, e.g., Crane (1995) for an instance of unwillingness to grant premiss (ii) above, that the only assumption which can reasonably be rejected is $(\mathrm{H})$.)
} 
the cause of the effect $e .^{38}$ The idea is that (CCP) (which is endorsed on the view in question) ensures - under the assumption that the holding of conditionals of the kind considered above is not only sufficient but also necessary for a property's causal efficacy - that there will be a physical property $P$ such that $\square$ $\left(\sim P c \rightarrow \sim P^{*} e\right)$. For any $M$ having $P$ as supervenience base, $\square(\sim M c \rightarrow \sim P c)$ will then hold. Hence, by transitivity, $\quad \square\left(\sim M c \rightarrow \sim P^{*} e\right) .{ }^{39}$

The problem, here, is that this 'causal conditionals/supervenience combination' (hereafter, CCS) does not, actually, seem enough to ensure causal efficacy. ${ }^{40}$ Examples can be conceived where (1) a property, ' $A$ ', has as a supervenience base another property, ' $B$ ', which is causally efficacious with respect to yet another property, ' $C$ '; (2) $A$ thereby satisfies the relevant conditional with respect to $C$; yet (3) $A$ is not causally efficacious with respect to

${ }^{38}$ Cf. LePore and Loewer (1987); Horgan (1989). (And Marras (1994); Zangwill (1996); ...) I am, however, making use of strict implications instead of conditionals à la Stalnaker. (And the association of causation with conditionals interpreted as implications having at least the same structure as strict implication is an option which some have, indeed, defended - e.g.: Burks (1951).) This is because - as it is going to emerge shortly - we need transitivity to derive causal efficacy from supervenience in the way to be considered, and transitivity does not hold for conditionals à la Stalnaker.

${ }^{39}$ The boxes of all these conditionals are, of course, assumed to correspond to the same set of possible worlds, which is the one in which a conditional must hold to ensure causal efficacy in accordance with the present view.

${ }^{40}$ Hence it fails to be an NHA by failing to satisfy condition (a) above. Condition (b), on the other hand, seems to be satisfiable given that, according to the account under discussion, there are differences between the relation holding between $M$ and $P^{*} e$ and that which holds between $P$ and $P^{*} e$. (On the grounds of these differences, the causal efficacy of $M$ with respect to $P^{*} e$ can be claimed to be different from that of $P$ with respect to $P^{*} e$.) 
$C$. So, for instance, consider the property being $r e d{ }^{41}$ This property can, in certain cases, be plausibly taken to supervene on the property being hot. ${ }^{42}$ In the case of two pieces of coal, for example, indistinguishability with respect to being hot entails indistinguishability with respect to being red; two pieces of coal cannot (ceteris paribus) differ as to whether they are red without, at the same time, differing as to whether they are hot. Hence, if a piece of coal which is both hot and red causes the smouldering of a tissue, and the coal's being hot is causally efficacious with respect to the tissue's being smouldered, we'll have a conditional of form $\square(\sim R x \rightarrow \sim S y)$, where $x$ stands for the piece of coal, $y$ stands for the tissue, $R$ stands for the property of being red, and $S$ stands for the property of being smouldered. Yet, the property being red does not seem to be causally efficacious with regard to the smouldering of the tissue.

One could, however, be dissatisfied with this being a counterexample to the claim that CCS is sufficient for causal efficacy. The property being red - one could argue - fails to be causally efficacious with regard to the smouldering of the tissue only because its supervenience on the property being hot holds only in specific cases (e.g. when the object is a piece of coal) and under ceteris paribus conditions. Indeed, one could even claim that, given that the covariation between the property of being red and that of being hot holds only in specific cases, the property being red is better regarded as being supervenient not on the property

\footnotetext{
${ }^{41}$ The example discussed here is taken from Segal \& Sober (1991). Segal and Sober, however, do not introduce it as an instance of supervenience. They use the example to undermine counterfactual criteria for causal efficacy.

${ }^{42}$ Given that supervenience is a relation between families of properties, this will be a limiting case where the families in question are constituted by single properties.
} 
being hot alone, but on the combination of the property being hot with any of the properties corresponding to the specific cases in which the covariance holds e.g. the property of being a piece of coal, or the property of being a bar of iron, or... If so, the failure of the property being red to be causally efficacious with respect to the smouldering of the tissue cannot be offered as a counterexample to the claim that CCS is sufficient for causal efficacy. For it cannot, properly speaking, be said to supervene on the $P$ in question. If, on the other hand, we had a case where the supervenience of the property whose causal efficacy is at issue on a causally efficacious property is not limited to specific cases and to the holding of ceteris paribus conditions, then the possibility of grounding the causal efficacy of the Mental on CCS would have been left open.

I think, though, that there are problems even when we do consider cases where the supervenience of the property whose causal efficacy is at issue on a causally efficacious property is not limited to specific cases and to the holding of ceteris paribus conditions. To see that, consider the property weighing more than 10 pounds or being a peanut. ${ }^{43}$ This property logically supervenes on the property having a particular mass $m$ and being on Earth via its logical supervenience on the property weighing 170 pounds. ${ }^{44}$ The property having a particular mass $m$

\footnotetext{
${ }^{43}$ Again, I am drawing from Segal \& Sober (1991).

${ }^{44}$ Again, we have here a limiting case of families of properties constituted by single properties. Moreover, it is important to point out that the domain over which the properties are distributed is assumed to be constituted by particulars which are not peanuts. If it weren't constituted by particulars which are not peanuts, the property of weighing more than 10 pounds or being a peanut (call it ' $A$ ') would fail to be logically supervenient on the property having a particular mass $m$ and being on Earth (call it ' $B$ '), as it would be possible for two particulars to be indiscernible in $B$ (e.g. none of the particulars in question has $B$ ) while being discernible in $A$
} 
and being on Earth is causally efficacious with respect to a scale's reading ' 170 '. Yet, it does not seem right to regard the property weighing more than 10 pounds or being a peanut as causally efficacious with respect to the scale's reading ' 170 '; the property weighing more than 10 pounds or being a peanut thus fails in causal efficacy although it logically supervenes on a causally efficacious property.

Similarly for the property being an abrupt utterance of the word 'hello'. ${ }^{45}$ This property logically supervenes on the property being an abrupt and loud utterance of the word 'hello'. Yet, it is not causally efficacious with respect to the heeding by someone who is hard of hearing. Once again, we have supervenience without causal efficacy. ${ }^{46}$

(e.g. both particulars do not weigh more than 10 pounds, but one of the particulars is a peanut, whereas the other isn't - hence one of the particulars has $A$ whereas the other doesn't).

${ }^{45}$ This time I am drawing (with modifications) from Menzies (1988).

46 This latter example, however, could be argued not to fit the pattern required for making of it a counterexample to the claim that CCS is sufficient for causal efficacy. As it will be remembered, in fact, the property which, in the pattern, is assumed to be causally efficacious with respect to the effect, is the property on which the allegedly inefficacious property supervenes. This - it might be argued - is not the case in the example which has just been considered. (The causally relevant property to the heeding by the person hard of hearing is that of being a loud utterance of the word 'hello', not that of being a loud and abrupt utterance of the word 'hello'.)

Does that mean that the example in question cannot be used as a counterexample to the claim that CCS is sufficient for causal efficacy? Well, it does iff it is true that the example does not fit the pattern. I am not sure about whether this is so. (After all - one might argue - why cannot the property of being a loud and abrupt utterance be regarded as causally efficacious with regard to the person's paying attention? Something like what, in Yablo (1992), features as the notion of 'being required' seems, here, to be at work; still, should we endorse such kind of principle?). So, I'll here leave the matter open. (Note that the same conclusion should be reached with regard to another prima facie counterexample to the claim that CCS is sufficient for causal efficacy. The 
I'll now try to support the claim that those accounts which stand a chance of being NHAs entail, in addition to MPS, at least one of the Assumptions. I'll be doing that by providing examples.

First, consider Segal and Sober's criterion of causal efficacy ${ }^{47}$ :

(P5) If (i) it is a (possibly nonstrict) law that every $F$ event causes a $G$ event and (ii) in each case in which an $F$ event causes a $G$ event there exist microproperties $m(F), m(F)^{\prime}$ and $m(G)$ such that the cause's being $F$ mereologically supervenes on its being $m(F)$ and the effect's being $G$ mereologically supervenes on its being $m(G)$ and possession of $m(F)$ includes possession of $m(F)^{\prime}$ ' and the cause's being $m(F)$ ' causes the effect's being $m(G)$, then $F$ is efficacious in the production of $G s$.

The notion of mereological supervenience is to be understood in terms of the

prima facie counterexample - credited to Lewis in Menzies (1988) - is that of the property being opaque, which can be taken to supervene on a categorical basis shared with the property of being an electrical conductor, and yet is causally inefficacious with respect to the property of being a death by electrocution. As before, the counterexample could be resisted by claiming that the causally efficacious property in the example is not the one constituting the supervenience base for the allegedly inefficacious property, but, rather, another property (i.e., in the present case, being an electrical conductor) which shares its supervenience base with this latter. Once again, however, I have uneasy feelings about the uncontroversial acceptability of the claim - this time because of the complexities attached to the issue of the relation between dispositions and categorical bases.)

${ }^{47}$ Segal \& Sober (1991), p. 15. The criterion is, in Segal and Sober's paper, referred to as '(P5)' because it is the fifth proposal which they consider. 
following definition ${ }^{48}$ :

(MS) $F$ mereologically supervenes on $m(F)$ iff it is nomologically necessary that if any object, $x$, has $m(F)$ at any time, $t$, then $x$ has $F$ at $t$.

$F$ stands for a macroproperty; $m(F)$ stands for a micro(based)-property. (A micro-based property is a property which can be characterized as having parts which have determinate properties and relations. A micro-based property is physical iff the corresponding parts are physical.) Thus, for mental properties to be causally efficacious in accordance with (P5), it is (given (CCP)) required not only that mental properties be supervenient on physical properties, but also that they can be regarded as macroproperties whose corresponding microproperties are physical. (And this commits one to $A 1$ : macroproperties are properties of wholes, and 'the corresponding microproperties' are the properties of the parts of the wholes in question.) Moreover, Segal and Sober claim that 'If $F$ mereologically supervenes on $m(F)$, then $x$ 's being $m(F)$ realizes $x$ 's being $F^{\prime}{ }^{49}$ From that it follows that for mental properties to be causally efficacious in accordance with (P5), it is also necessary - on Segal and Sober's view, and, again, given $(\mathrm{CCP})$ - that mental properties be realizables having physical

\footnotetext{
${ }^{48}$ Segal and Sober (1991), p. 10.

${ }^{49}$ Segal \& Sober (1991), p. 10.
} 
properties as their realizers. $^{50}$

The idea that mental properties are realizables having physical properties as their realizers can, actually, by itself, provide a way of arguing for the causal efficacy of the mental. This is, indeed, what seems to be happening in chapter 18 of Kim (1993). There, in fact, it is argued that in case mental properties could be taken to be of the kind of properties which can be realized, and efficacious physical properties had to be regarded as their realizers, CEM would follow. ${ }^{51} \mathrm{I}$ thus suggest to take Kim's proposal as another candidate NHA which, in addition to MPS, entails one of the Assumptions. ${ }^{52}$

Finally, let me cite Macdonald \& Macdonald (1986) and Yablo (1992) as further instances of such candidate NHAs. In both cases a central role is played by the idea that mental properties can be regarded as 'determinables' having physical properties as their 'determinates'. However, what goes under the name of 'the relation between a determinable and its determinates' is not meant to entail a form of supervenience characterized by conceptual necessity. Accordingly, it is better to take the Macdonalds' and Yablo's proposals as cases

${ }^{50}$ On the basis of what Segal and Sober write, it is not possible to establish whether they would be happy to speak of a relation of realization also in cases where the relation in question is associated with a two-operator version of supervenience where the first modal operator ascribes metaphysical (as opposed to physical) necessity. This kind of indeterminacy is quite common in the literature. Accordingly, it has been preserved in the definition of $A 3-R / R$ so as to make it possible to associate this latter with the notion of realization as used in the literature.

${ }^{51}$ This line of thought emerges also in Kim (1997a, 1997b, 1998).

${ }^{52} \mathrm{Kim}$ 's proposal can be taken to be a candidate NHA insofar as it is an account which aspires to be a satisfactory account of the causal efficacy of the mental, and which, given the assumption of (CCP), (CEE), and (PD), is - on the line of argument considered at the beginning of the section - to be taken to aspire to being compatible with a rejection of $(\mathrm{H})$. 
where what is required to have CEM is, in addition to mere psychophysical supervenience, $A 3-R / R$ rather than $A 3-D / D$. (That what is required is $A 3-R / R$ is actually controversial in the case of Yablo (1992), because of Yablo's lack of stand as to whether the mental/physical relation is to be associated with a twooperator version of supervenience. If not $A 3-R / R$, however, it is still some version of $A 3$ that is required for having CEM in accordance with Yablo's proposal.)

Let us now turn to the exception to the 'rule' that any account which stands any chance of being an NHA entails, in addition to MPS, at least one of the Assumptions. This exception provides a picture which - contrary to what happens with CCS - is not obviously inadequate as an account of CEM. ${ }^{53}$ According to this picture (which assumes (CCP), (CEE), and (PD)), the mental is claimed to be causally efficacious not only because, in virtue of its supervenience on the physical, it turns out to be necessary for the effect which one is considering, but also because it turns out to be sufficient. Given (CCP), (CEE), and (PD), the mental can be sufficient for the effect in question only by necessitating the physical properties which are causally efficacious with respect to the effect. Hence, in addition to psychophysical supervenience, it is required that the mental necessitate the physical properties which are causally efficacious

\footnotetext{
${ }^{53}$ I am, once again, granting, perhaps, quite a lot, by presenting the picture under discussion as not obviously inadequate as an account of CEM (and, hence, as an account which stands some chance of being an NHA). In case the picture under discussion were obviously inadequate as an account of CEM, the job of proving ACEM inadequate to counterexemplify (Th) would be even easier.
} 
with respect to the effect in question. ${ }^{54}$

The point is that this requirement (call it ' $R$ ') can be satisfied without making any of the Assumptions. Indeed, the satisfaction of $R$ is - for reasons which will emerge in $\S 4.6$ - incompatible with at least some of the Assumptions. We thus have a candidate NHA which does not entail any of the Assumptions; we have gained the possibility of arguing for MPS via ACEM without commitment to the Assumptions.

However, although the satisfaction of $R$ does not commit one to the Assumptions, it is still likely that one is going to end up committed to assumptions of at least an empirical nature when the picture offered by our candidate NHA is to be completed by giving an account of the alleged satisfaction of $R$. For, given that the satisfaction of $R$ is not to be based on any of the Assumptions, the issue then arises of providing an alternative ground. And it is difficult to see how the satisfaction of $R$ can be grounded if, in addition to the Assumptions, one rejects also the possibility of accounting for the satisfaction of $R$ by appealing to claims such as, for instance, the one that repeated observations of psychophysical correlations make it reasonable to believe in psychophysical connections which hold as a matter of necessity. So, the only case where, by way of an instance of ACEM, it is possible to support MPS without committing oneself to the Assumptions, appears to be a case where some empirical claims

${ }^{54}$ Cf. Horgan (1987). Our 'necessitations' correspond to psychophysical correlations which are assumed to hold in the actual world. Psychophysical supervenience can then give modal force to these correlations. The idea is, further, that the causal inefficacy of the mental would be avoided in virtue of the fact that conditionals of the form $(P c \& \sim Q c)>e$ and $(\sim P c \& Q c)>\sim e$ (where $c$ stands for a cause; $e$ stands for an effect; $P$ stands for a physical property; and $Q$ stands for a mental property supervenient on $P$ ) would have been made to hold vacuously. 
need to be embraced instead. But, in accordance with what has been argued in the previous section, this kind of claims can be maintained to have a chance of being satisfactorily supported only for some mental states. What can be got here is not, then, MPS in its full generality. This, together, with the rest of what has been argued in this section, means that MPS in its full generality can't be got, via ACEM, without commitment to at least one of the Assumptions. Once again, it has not been possible to counterexemplify (Th). 


\section{Reduction}

\subsection{Supervenience as reductive}

What has been argued in chapter 2 points to one way in which an MPS claim can be strong: An MPS claim can be strong by being an SSS claim (i.e. a claim of supervenience as satisfactorily supported). For, given (Th) and the nature of the Assumptions, an SSS claim will be either a claim of a single relation which rules out more possibilities than the ones which are ruled out by supervenience alone (when considered independently of its support), or a disjunctive claim having relations of this kind as disjuncts. (Cf. $\S 2.1$.) In $\S 3.4$ I'll provide an illustration of this point. But what I want to do, more generally, in this chapter is to consider another way in which an MPS claim can be strong; I shall consider the possibility of an MPS claim being strong to the extent that it entails psychophysical reduction.

This 'way of being strong' is, of course, related to the 'way of being strong' considered in the previous chapter, if there are 'reductive requirements' which can be fulfilled by a supervenience claim only if it is an SSS claim. This is, in fact, what I said I'll be considering in $\S 3.4$. More generally, however, I'll argue that, at least under certain notions of reduction, there are forms of supervenience 
which entail reduction - regardless of any consideration concerning their support.

This is the gist of $\S 3.2$ and $\S 3.3$. In these sections, though, I'll also be concerned with showing how, in some cases, the yielding of reduction by supervenience can hold only within metaphysical frameworks which are not congenial to all. It will thus begin to emerge to what extent my position is to be distinguished from that of an Enthusiast. (Cf. the Introduction.) In $\S 3.4$, on the other hand, I will, once more, provide reasons for resisting the Sceptic, as my main concern is going to be that of showing how some forms of supervenience are, in virtue of their 'reductive potential', inconsistent with Anomalous Monism and Psychophysical Emergentism - i.e. two significant positions in the debate on the Mind-Body problem.

\subsection{Theory reduction and explanation}

The obvious place to start a discussion of reduction is with Nagel's model. ${ }^{1}$ According to it, reduction is to be understood in terms of the derivation of the laws of a branch of science from the laws of another branch of science and some 'bridge principles' relating the terms of the former to the terms of the latter.

\footnotetext{
${ }^{1}$ See Nagel (1961), ch. 11.
} 
Contrary to what it has at some point been assumed ${ }^{2}$, these 'bridge principles' are not required to be biconditionals. ${ }^{3}$ It is important to note, however, that when the 'bridge principles' are mere conditionals, derivability is not ensured. For from the possibility of establishing conditionals which relate the terms of one branch of science to those of another, it does not follow that the former branch of science will contain 'images' of the laws of the latter, and, hence, that it will be possible to derive the latter from the former by means of the conditionals in question. By contrast, the existence of biconditionals between the terms of one branch of science and those of another, does ensure the derivability of the laws of one branch of science from the laws of the other. For in case some of the laws of the branch of science which we want to reduce have no 'images' in the branch of science which is meant to be the reducer, we can, by using the biconditionals in question, 'translate' the laws under discussion into laws which can be formulated by using terms of the branch of science which is meant to be the reducer. It will thus be possible to derive the branch of science which we want to reduce from at least an enriched version of the branch of science which was

${ }^{2}$ Cf., e.g., Kemeny \& Oppenheim (1956). (Kemeny \& Oppenheim's discussion of Nagel's model, however, is based on Nagel (1951) rather than Nagel (1961).) Another example is Kim (1978). Given, however, Kim's position in more recent writings, it is, maybe, a bit unfair to cite him as an example in this context.

${ }^{3}$ Cf. Nagel (1961), p. 355, note 5: '[...] the linkage between $A$ [standing for the terms of the branch of science which is reduced] and $B$ [standing for the terms of the branch of science which is the 'reducer'] is not necessarily biconditional in form, and may for example be only a one-way conditional: If $B$, then $A[\ldots]$ '. 
meant to be the reducer. ${ }^{4}$

This acquires a particular importance within our context. What supervenience can ensure is the possibility of establishing conditionals between the subvenient and the supervenient. Taking the subvenient and the supervenient to be sets of terms of branches of science, it is then also possible to have - as Kim has shown - biconditionals between disjunctions of terms of one branch of science and terms of another branch of science, as something that supervenience can ensure. ${ }^{6}$ Disjunctions of terms of a branch of science, however, are not themselves, necessarily, terms of that branch of science. (At least some disjunctions of terms of a branch of science do not, definitely, seem to be themselves terms of that branch of science - if 'terms of a branch of science' means 'predicates featuring in the laws of the branch in question'. So, for example, consider the disjunction

\footnotetext{
${ }^{4}$ Cf. Nagel (1961), p. 355, note 5. In Nagel the point is made by distinguishing between 'derivability' and 'connectability' (by which it is meant the possibility of establishing 'bridge principles' between the terms of the two branches); connectability entails derivability if and only if the bridge principles are biconditionals.

${ }^{5} \operatorname{Kim}(1978 ; 1984 a)$.

${ }^{6}$ In case the sets are closed under conjunction and complementation, it won't be possible to ensure that what can be had are disjunctions of terms of branches of science as opposed to disjunctions of (mere) conjunctions and complements of terms of branches of science. In the main text, I am thus taking 'supervenience' to stand for forms of supervenience whose sets are not closed under conjunction and complementation.
} 
having mass $M$ or having energy $E$ or being the magnetic field $M F.)^{7}$ This does not mean that there can't be disjunctions of terms of a branch of science which are themselves terms of that branch of science. But it does mean that not all disjunctions of terms of a branch of science are themselves terms of that branch of science. And given that there does not seem to be any feature of supervenience which could ensure that the disjunctions entailed by the supervenient are such that they can be regarded as themselves terms of the subvenient branch of science, it follows that supervenience can't ensure that we can establish biconditionals between terms of branches of science. ${ }^{8}$ In the light of what has been said about 'bridge principles', this means that supervenience cannot ensure the satisfaction of Nagel's model. ${ }^{9}$

Nagel's model, however, is meant to apply to branches of science. So, to say that supervenience can't ensure the satisfaction of Nagel's model is to say that,

\footnotetext{
${ }^{7}$ This seems to be so even if the idea is that disjunctions of terms of a branch of science are, if not themselves terms of that branch of science, at least equivalent to terms of that branch of science.

${ }^{8}$ The line of argument which has been sketched in this paragraph will be developed in much more detail in the next section when we'll be dealing with disjunctions of entities other than terms of branches of science. The sketch which has been given should, hopefully, be sufficient for the present purpose.

9 Unless, of course, supervenience could ensure derivability other than by ensuring biconditionals between terms of branches of science. Given, however, that the forms of supervenience which we are considering do not have laws as relata, it is hard to see how supervenience could ensure derivability other than by ensuring biconditionals between terms of branches of science.
} 
under this model, supervenience can't ensure the reduction of branches of science. But what about the relata of the supervenience relations which are the object of our discussion? What about properties, for instance? Is there anything which can be said in the present context, when what we are considering are properties as opposed to branches of science?

Nagel allows for a reduction of properties to the extent that this latter is understood as parasitic on reduction of theories. ${ }^{10}$ For, he argues, there are no properties independent of theories which are used to define them. And whether the properties studied by one science are reducible to the properties studied by another depends on whether the theory corresponding to the former is reducible to the theory corresponding to the latter. ${ }^{11}$

Thus, on this view, supervenience fails to ensure property reduction. For, as we have seen, supervenience fails to ensure theory reduction, which, on this view, is required for having property reduction.

There is, however, also a quite different moral which can be drawn from some of Nagel's more general claims. At p. 338, for example, he claims that

\footnotetext{
${ }^{10}$ I have here shifted from talking of branches of science to talking of theories. This does not make any difference for both 'branches of science' and 'theories' can be understood as sets of laws.

${ }^{11}$ Nagel (1961), pp. $364 \mathrm{ff}$. The idea that property reduction is parasitic on theory reduction is, then, present also in what can be regarded as developments of Nagel's model. (Cf., e.g., Churchland (1986), where property reduction is understood as the relation which holds between the properties of two theories when one is reducible to the other.)
} 
'[...] reduction [...] is the explanation of a theory or a set of experimental laws established in one area of inquiry, by a theory usually though not invariably formulated for some other domain.' This suggests the yet more general idea of understanding reduction in terms of the explanation by the reducer of what is reduced. ${ }^{12}$ Moreover, Nagel's model can - as it will be clear shortly - be regarded as a special case of Kemeny and Oppenheim's model, where reduction is understood in terms of the explanation by the reducer of all that is explained by what is reduced..$^{13}$ That provides us with two ways of conceiving of reduction which are independent of what is taken as the reducer and of what is taken as what is reduced. As such, these two ways of conceiving of reduction can be of interest in the present context, where what are being considered are properties rather than theories. ${ }^{14}$

In both Nagel's and Kemeny \& Oppenheim's model, explanation is understood in terms of derivability: $A$ explains $B$ iff $B$ is derivable from $A$. The notion of 'derivability' is then understood as follows: $B$ is derivable from $A$ iff we can get $B$ as a result of a general premiss, such as that to the effect that

\footnotetext{
${ }^{12}$ Cf. Hempel (1969).

${ }^{13}$ Kemeny \& Oppenheim (1956), p. 13 ff.

14 These two ways of conceiving of reduction are still present in more recent models. In particular, then, they are still present in those models where instead of requiring that the theory to be reduced be deducible from the reducer, all that is required is that it is a theory standing in an 'analogue relation' with the theory to be reduced that is to be deducible from the reducer. (See, e.g., Hooker (1981); Churchland (1986).)
} 
whenever we have $A$ we have $B$, and of the assumption that we have $A .^{15}$

In Nagel's model, the theory to be reduced is thus explained by the reducer to the extent that it is derivable from the reducer. Under the conception of explanation as derivability, whatever is explainable by the theory to be reduced is then going to be derivable from this latter. Given that derivability is transitive, it follows that when $T_{1}$ explains $T_{2}$, whatever is explainable by $T_{2}$ is going to be explainable by $\mathrm{T}_{1}$.

When NCMOFs of MPS hold ${ }^{16}$, we have, for every instantiation $F x$ of a mental property $M$, an instantiation $G x$ of a physical property $P$ and a universal conditional $(\forall \mathrm{y})(\mathrm{Gy} \rightarrow \mathrm{Fy})$ which yields $F x$ as a result of $G x$. To this extent, we can say that psychophysical supervenience can yield psychophysical derivability - i.e. the derivability of the mental from the physical. Under the conception of explanation as derivation, psychophysical supervenience can thus ensure that the

${ }^{15}$ Cf. Hempel \& Oppenheim (1948). Nowdays there is, of course, widespread scepticism about this being an adequate account of explanation. On the other hand, though, it must also be noted that there is no received alternative.

I'd then like to draw the reader's attention also to the compatibility between taking explanation to be asymmetric and taking the condition for derivability stated above to be satisfied on the basis of a relation which is not asymmetric. Explanation can be taken to be asymmetric even when our condition for derivability is satisfied on the basis of a relation which is not asymmetric (e.g. $A \leftrightarrow B$ ), in virtue of the asymmetry introduced by stipulating what is to count as the deriver (i.e., in our case, $A$ ). The import of that will be clear as a result of what will be argued in the next chapter, when considering the issue of the relationships between supervenience, asymmetry, and explanation. 
mental is explainable by the physical. If, then, reduction is conceived of in terms of the explanation of what is reduced by the reducer, it follows that psychophysical supervenience can ensure the reducibility of the mental to the physical. And if explanation is transitive, it will be possible to conclude that psychophysical supervenience can ensure the reducibility of the mental to the physical also when reduction is conceived of in terms of the explanation by the reducer of all that can be explained by what is reduced.

There are, then, at least two conceptions of reduction (i.e. reduction as explanation by the reducer of what is reduced, and reduction as explanation by the reducer of all that is explained by what is reduced) under which psychophysical supervenience can entail psychophysical reduction. In order for that to be the case, however, the notion of 'explanation' is to be understood in terms of 'derivability'. In the next section I'll consider other conceptions which lead to the yielding of psychophysical reduction by some forms of psychophysical supervenience. And similarly to what has happened in this section, it will also be argued that, in some cases, psychophysical supervenience can entail psychophysical reduction only under certain assumptions.

\footnotetext{
${ }^{16}$ To remind the reader: 'NCMOFs' stands for 'Modal Operator Forms of supervenience whose sets are not closed under conjunction and complementation'. (See $§ 1.2$.)
} 


\subsection{Ontological reduction}

The previous section started with a discussion of theory reduction. What we did was then to apply to entities such as properties some general ideas which could be extracted from models of theory reduction. Taking a realist view of properties, this move would be put by some as a shift from a discussion of theory reduction to a discussion of ontological or metaphysical reduction - the general idea behind this distinction being that of a distinction between, on the one hand a form of reduction whose relata are linguistic entities, and, on the other, a form of reduction whose entities are mind-independent. ${ }^{17}$ In this section, I want to carry on my discussion of ontological reduction by considering its association with the idea that what is reduced is nothing over and above its reducer ${ }^{18}$ As we are going to see, there are different ways in which the notion of being nothing over and above can be understood. I'll begin by considering one of them, and whether, under the conception in question, supervenience can entail reduction.

The first way of conceiving of the notion of ontological reduction/being nothing over and above that I want to consider (call it ' $\mathrm{C} 1$ ') is one according to which the $A s$ are reducible/nothing over and above the $B s$ iff all truths about the

\footnotetext{
${ }^{17}$ Cf., e.g., Van Cleve (1990); Charles (1992); Stalnaker (1996).

${ }^{18}$ Cf. Kim (1978); Enç (1986); Lewis (1994a); Stalnaker (1996); Jackson (1998).
} 
$A s$ are entailed by truths about the Bs. (Cf., e.g., Van Cleve (1990), p. 218.) This clearly corresponds to the notion of reduction as derivability considered in the previous section. The only difference is that in the present case the relata of the relation which yields reduction are not the same as the relata of the relation of reduction: the relata of the relation which yields reduction are truths concerning $A s$ and $B s$, whereas the relata of the relation of reduction are the $A s$ and $B s$ themselves. But this distinction is not, after all, that important. For, similarly to what happened in $\S 1.5$, the derivability of the $A s$ from the $B s$ yields (under the notion of 'derivability' in play in the previous section) the entailment of all truths about the $A s$ by truths about the $B s$, and this latter entailment yields the derivability of the $A s$ from the $B s$. So, as (a certain form of) supervenience could be shown to yield (a relevant kind of) derivability, it is, by the same token, also possible to say that supervenience can yield reduction under $\mathrm{C} 1$.

If the reducer is to be constituted by physical properties which are neither (mere) conjunctions nor complements of physical properties, the form of supervenience required to yield reduction under $\mathrm{C} 1$ is an NCMOF. Moreover, given that what is required is that all the $A$-truths be entailed by $B$-truths, the relevant form of supervenience features a strong necessity (i.e. a metaphysical or a conceptual necessity) as the kind of necessity ascribed by both the first and the second modal operator.

Under other conceptions (call them ' $\mathrm{C} 2 \mathrm{~s}$ ') which are put forward as notions 
of ontological reduction/being nothing over and above ${ }^{19}$, reduction can, by contrast, be yielded also by forms of supervenience which do not feature two modal operators ascribing a strong necessity. This is because, under $\mathrm{C} 2 \mathrm{~s}$, all that is required from some $A s$ to be reducible/nothing over and above some $B s$ is the holding of certain entailments between propositions involving $A s$ and $B s$ within a restricted set of possible worlds. When the $A s$ and the $B s$ in question are, respectively, the mental and the physical properties, what is required is, for example, the yielding of the complete description of the distribution of mental properties of our world by the complete description of the distribution of the physical properties of our world in any world which is a 'minimal physical duplicate of our world', or, again, in any world with no natural properties 'alien to our world' ${ }^{20}$ That allows for possible worlds which feature the same distribution of physical properties as the one of our world, and yet differ from our world in their distribution of mental properties because of the presence of, say, some non-physical particulars instantiating some mental properties. (This kind of possible worlds will be referred to as 'dualistic worlds'.)

\footnotetext{
${ }^{19}$ Cf., e.g., Lewis (1983a; 1994a); Jackson (1998).
}

${ }^{20}$ See Jackson (1998), and Lewis (1983a). A 'minimal physical duplicate of our world' is a world that "(a) is exactly like our world in every physical respect, and (b) contains nothing else in the sense of nothing more by way of kinds or particulars than it must to satisfy (a)." (Jackson (1998), p. 13.) A property is 'alien to a world' iff "(1) it is not instantiated by any inhabitant of that world, and (2) it is not analysable as a conjunction of, or as a structural property constructed out of, natural properties all of which are instantiated by inhabitants of that world." (Lewis (1983a), p. 364.) 
What is not clear, however, is whether one can, indeed, regard as a notion of reduction a conception, like $\mathrm{C} 2$, according to which mental properties can be taken to be reducible/nothing over and above physical properties even if the kind of possibility above is not barred. One can, of course, use the term 'reduction' as one wishes. If, however, we want to go beyond mere stipulations, then - it seems to me - some link must be preserved with the notion/s of theory reduction considered in the previous section, which is, after all, what the term 'reduction' originally corresponded to. And the problem is that it does not seem possible, as I am now going to show, to preserve any significant link with theory reduction if one allows for dualistic worlds. ${ }^{21}$

The existence of dualistic worlds, in fact, is inconsistent with all the requirements of theory reduction, when these latter are generalized so as to apply to mental and physical properties. It is, for instance, inconsistent with the explicability of the mental properties by the physical properties, even when by 'explicability' one doesn't mean anything more than 'derivability'. For in a dualistic world there are instantiations of mental properties which are not derivable from physical properties. The fact that these instantiations of mental properties can be used as explanantia can then be taken to undermine also the necessary explicability by physical properties of all that is explainable by mental

21 Note that $\mathrm{Cl}$ is not affected by similar problems; as we have seen, $\mathrm{Cl}$ actually corresponds to one of the general notions which can be extracted from the model/s of theory reduction considered. 
properties. And, finally, the existence of dualistic possible worlds undermines also the possibility of the mental properties being 'incorporated', qua entities to be reduced, in the physical properties. ${ }^{22}$ For the existence, in the dualistic worlds, of particulars which are mental particulars without being physical particulars, rules out the possibility of taking the set of the instantiations of mental properties to be a subset of the set of the instantiations of the physical properties.

Although the satisfaction of the requirement associated with C2s can be ensured by supervenience, it is still, thus, possible to argue that what supervenience can't thereby ensure is the satisfaction of a requirement which can, in any reasonable sense, be taken to give us reduction. For some, then, even the satisfaction of the requirement associated with $\mathrm{C} 1$ is not enough to give us reduction.

This is because it is possible to associate the notion of ontological reduction/being nothing over and above with a requirement which is (at least potentially) even stronger than the one associated with $\mathrm{C} 1$. It is possible to require for some entities $A s$ to be reducible to some entities $B s$, that the $A s$ be identical to the $B s$.

This might, prima facie, impose a requirement for which it does not make much sense to ask whether it can be satisfied by supervenience. For supervenience is supposed to be weaker than identity. Indeed, given that identity

\footnotetext{
${ }^{22}$ See Nagel (1961), p. 240; and Hempel (1969), p. 190 for the idea that what is reduced is
} 
ensures supervenience, the entailment of identity by supervenience would, arguably ${ }^{23}$, mean that supervenience could no longer be regarded as something different from identity.

I believe, however, that the situation is actually slightly more complicated than that. For one thing, one needs to distinguish - I think - between cases where we are dealing with particulars and cases where we are dealing with universals. And in case we are dealing with particulars, it might be that there are at least some versions of supervenience which yield identity. For, if a psychophysical version of an NCMOF holds, then any particular which has a mental property will have a physical property. And if for a particular to have a mental property is for it to be mental, and for a particular to have a physical property is for it to be physical, it then follows that the holding of a psychophysical version of an NCMOF implies that mental particulars are physical particulars. (Note that this can escape the worry about supervenience collapsing into identity insofar as the relata of the identity which can be ensured by supervenience are different from the ones of the supervenience on the basis of which the identity is ensured.)

This is, admittedly, unsatisfactory, to the extent that what might be required

\footnotetext{
'incorporated in'/ 'assimilated by' the reducer.

${ }^{23}$ I say 'arguably' because one might, by analogy with the case of properties, have, also in the case of relations, qualms about the possibility of taking mutual entailment as a condition which is sufficient for having identity.
} 
for, say, a mental particular to be nothing over and above a physical particular is, actually, that the mental particular be only a physical particular. Then, probably, everything would, indeed, hinge upon whether mental properties are nothing over and above physical properties. (For if mental properties were nothing over and above physical properties, it would be hard to see how a particular could be something over and above a physical particular in virtue of being a mental particular.) In this case my contention is that whether it can make any sense to speak of supervenience as yielding identity depends on the notion of property in play.

In order to show that, let me begin by making some remarks on what can be taken to follow from some very general features of identity and supervenience. For certain types of entities (e.g. properties) a necessary condition of identity is coextension of the corresponding predicates. What supervenience can ensure is coextension between predicates and disjunctions of predicates. (Cf. § 3.2.) But what identity requires is also, by Leibniz's Law, indiscernibility of the relata. In particular, then, if one of the relata has the second-order property of being a property, then, for something to be identical with it, this something too must have the second-order property of being a property. That means that in the case of supervening properties, one can have identity only if the disjunctions of subvening predicates which are yielded by supervening predicates pick out properties. And the issue of whether supervenience can yield identity can be put 
in terms of whether the disjunctions of properties which are yielded by supervenient properties are themselves properties.

Now suppose that all that it takes to be a property is to be a set of particulars. Then, disjunctions of properties would themselves be properties, since a disjunction of properties would correspond to a union of sets, and a union of sets is itself a set. Moreover, under this way of conceiving of properties, predicatecoextension is sufficient for property-identity. ${ }^{24}$ Hence, supervenience can yield property-identity when properties are conceived of as being nothing but sets of particulars. ${ }^{25}$ (And, in the psychophysical case, the identity yielded will relate mental properties to physical properties, if the notion of 'physical property' in play is one according to which a property can count as physical as long as it is instantiated exclusively by particulars possessing properties which are used in

\footnotetext{
${ }^{24}$ We are here, of course, speaking of necessary coextension. Accordingly, the relevant kinds of supervenience will be forms of strong supervenience whose sets are not closed under resplicing (i. e. the 'NRSS' of $\S 1.4$ ). Moreover, to have property-identities we need predicate-coextensions which hold in all possible worlds. Another set of distinctions that acquires importance in the present context is thus that concerning the type/s of necessity of forms of supervenience. (Cf. $\S$ 2.2. More on that, anyway, in the Conclusion.)

25 Note that, as before, it does not, still, follow that supervenience collapses into identity: If a set of properties $A$ is identical to a set of properties $B$, the set of properties $A$ is supervenient on the set of properties $B$. Supervenience would collapse into identity only if the identity of $A$ with $B$ were equally yielded by the supervenience of $A$ on $B$. And this is not what follows from what has been argued; the only identity that can be derived from the supervenience of $A$ on $B$, in accordance with what has been argued, is the identity between members of $A$ and disjunctions of members of $B$.
} 
the physical sciences ${ }^{26}$.)

Under other ways of conceiving of properties, however, it is no longer the case that supervenience can yield property-identity. In particular, then, what is no longer the case is that all disjunctions of properties which are entailed by supervenient properties are themselves properties.

This is true - I think - under quite a few ways of conceiving of properties. Rather than arguing for that, however, by mere 'case-by-case' consideration, I suggest to approach matters by considering, instead, whether there is anything

${ }^{26}$ Cf. Hellman \& Thompson's notion of 'ontological status' of properties, as in the following passage:

Confusion threatens when a property or attribute is called "material" or "mental" without specification of whether it is the ontological status or rather the ideological status that is in question. If it is the former, the matter turns primarily on the ontological status of the objects possessing or exemplifying the property. [...] If it is the latter, at issue is the $k i n d(s)$ of predicates (from what scientific discipline or domain of discourse) expressing the property. Thus, for a paradoxical sounding but extremely important example, a property may have the ideological status of being mental but not physical and yet have the ontological status of being physical but not mental! On our view, this happens whenever any psychological predicate $P$ is such that it is not lawlike coextensive with any physical predicate: the attribute expressed by $P$ is mental but not physical (ideologically), but given the Principle of Physical Exhaustion'*!, IT IS PHYSICAL BUT NOT MENTAL (ONTOLOGICALLY) IN VIRTUE OF BEING POSSESSED SOLELY BY PHYSICAL OBJECTS. [Hellman \& Thompson (1977), p.318. ( The capitalization is mine.)]

* The essential idea of the Principle of Physical Exhaustion (or Ontological Physicalism) is put as follows:

Our ontology includes at the very least all concrete referents of the terms of basic physical theory. In addition it includes every part or sum of parts of the entities initially accepted. Finally, our mathematical-physical ontology includes every object occurring at any level of an ordinary set-theoretic hierarchy taking as urelements the null set and the entities already recognized. The principle of Ontological Physicalism holds that the universe so delineated embraces everything there is. [Hellman \& Thompson (1977), p. 310.] 
general which can be said about how these different ways of conceiving of properties can lead to the conclusion that not all the disjunctions of properties which are entailed by supervenient properties are themselves properties.

A very general way of conceiving of properties, which can be taken to subsume as special cases many more specific ways of conceiving of properties, is the idea of a property as an entity to be defined in terms of its fulfilment of a determinate role. ${ }^{27}$ That opens the possibility - I suggest - of arguing against identifications of disjunctions of properties with properties by either arguing that in order to fulfil the role in question it is not necessary to introduce properties other than the ones corresponding to disjuncts, or by arguing that, at least in some cases, there is no role whose fulfilment would require us to introduce in our ontology a property corresponding to the disjunction at issue.

To illustrate the first strategy, I suggest to take the idea of properties as truthmakers. ${ }^{28}$ Under this way of conceiving of properties, disjunctions of properties can be regarded as properties only if the introduction of entities other than the ones corresponding to disjuncts is required to ground the truth of determinate expressions. Yet, even in the case of disjunctive expressions - which, if any, would be the expressions whose truth is grounded in the disjunctions of properties - it does not seem necessary to introduce entities other than the ones

\footnotetext{
${ }^{27}$ Cf. Lewis (1986b), esp. pp. 55 ff. (And Oliver (1996).)

${ }^{28}$ See Armstrong (1978); Lewis (1986b); Martin (1996).
} 
corresponding to the disjuncts in order to have an appropriate ground for truth. For any of the properties corresponding to the disjuncts can be taken to ground the truth of the corresponding disjunct; and once we have one of the disjuncts, we also have, by Disjunction Introduction, the disjunction itself. (E.g.: the possession of the property $F$ by $x$ can be taken to ground the truth of the sentence ' $x$ has $F$ '. But once the truth of $F x$ has been grounded, then, by Disjunction Introduction, also the truth of, say, $F x \vee G x$ will have been grounded. There is thus no need to appeal to any further property.) From that it follows that disjunctions of properties can never be regarded as themselves properties, under the idea of properties as truth makers. ${ }^{29}$

The conclusion that disjunctions of properties can never be regarded as themselves properties is actually stronger than the one that is needed to prove on the basis of the considerations above that supervenience cannot yield identity. For all that is needed to prove this is the idea that not all disjunctions of properties are themselves properties - combined with the idea that the mere fact of being disjunctions of subvenient properties cannot ensure that the disjunctions in question won't be among the ones which are not themselves properties.

The conclusion that not all disjunctions of properties can themselves be regarded as properties, can be reached by way of the second strategy above. This, as it will be remembered, rested on the idea that, at least in some cases,

\footnotetext{
${ }^{29}$ Cf. Armstrong (1978), vol. II, pp. 20 ff. My way of putting things, however, differs from
} 
there is no role whose fulfilment would require us to introduce in our ontology a property corresponding to the disjunction at issue. As I am now going to argue, the conception of properties as grounds of objective resemblances ${ }^{30}$ can be taken as one way of making this claim more determinate, and, arguably, acceptable.

If, in fact, properties are conceived of as grounds for objective resemblances among things, it becomes plausible to believe that for at least some disjunctions (e.g. being a mass $v$ being a velocity $v$ being a magnetic field) there is no corresponding property to the extent that there is no objective resemblance to ground..$^{31}$ Admittedly, the notion of 'objective resemblance' is vague. ${ }^{32}$ That, however, does not affect the present issue to the extent that it seems plausible to believe that there are always going to be some determinate notions of 'objective resemblance' under which it is going to be true that in the case of at least some disjunctions there is no corresponding objective resemblance to ground.

Under the conception of properties as grounds for objective resemblances, it will thus not be the case that supervenience can yield identity unless the fact that a disjunction of properties is a disjunction of subvenient properties can ensure

\footnotetext{
Armstrong's to the extent that I am not putting things in terms of correspondence-rules.

${ }^{30}$ See Armstrong (1978).

${ }^{31}$ Cf. Armstrong (1978), vol. II, p. 20. Note that the mere existence of an objective resemblance among individuals instantiating a disjunction is not going to be enough to make of the disjunction a property. For the resemblance in question might well not characterize only the individuals instantiating the disjunction. (The corresponding property would not, in this case, be associated with the disjunction.)
} 
that the disjunction in question is among the ones which correspond to some objective resemblance.

Now one could maintain that there are objective resemblances corresponding to disjunctions of subvenient properties to the extent that these disjunctions are entailed by properties (i.e. the supervenient properties).

But within our context, that is going to be of no help. For what we are interested in is the reduction of the supervenient to the subvenient. And that means that the objective resemblance in question must be a resemblance in the subvenient. (Example: the individuals in question must resemble in a physical respect if what we are interested in is the reduction of the mental to the physical.) $)^{33}$

Similar considerations apply to the case of properties as grounds for causal powers. ${ }^{34}$ In this case, one can argue that not all disjunctions of properties are themselves properties to the extent that in at least some cases there does not seem to be any causal power which is new (in the sense of not being identifiable with any of the causal powers of the individual disjuncts) and which can be associated with the disjunction in question. ${ }^{35}$ Our dealing with disjunctions of properties which are subvenient properties would, then, be of no help. For it is

\footnotetext{
${ }^{32}$ Cf. Oliver (1996), p. 30-1.

${ }^{33}$ Cf. Fodor (1974); Teller (1984b).

${ }^{34}$ Again, see Armstrong (1978).

${ }^{35}$ Cf. Armstrong (1978), vol. II, p. 20.
} 
hard to see how the fact that the properties in question are subvenient properties can guarantee that their disjunctions are associated with new causal powers. ${ }^{36}$

To that, however, it could be objected that the idea of not having a new causal power whenever we have a disjunction is actually quite controversial. If, for example, one conceives of causal laws as causal explanations, and of causal powers as causal explanantia, one could take disjunctions to be always associated with new causal powers to the extent that disjunctions allow for the possibility of explaining disjunctive explananda. ${ }^{37}$

A proper assessment of this move would require the consideration of issues which, in reason of their broadness and complexity, cannot be considered here. What I can do, instead, is to point out that even if all disjunctions are associated with causal powers which are different from those of the individual disjuncts, it might still be possible to argue against taking disjunctions of properties as themselves properties by adopting, also in the present case, a version of the first strategy. For when the causal powers of one of the disjuncts have been grounded, we'll have the disjunct, and, by Disjunction Introduction, also the disjunction itself. If, then, the disjunction itself cannot be had unless the

\footnotetext{
${ }^{36}$ And to appeal to the causal power of the supervenient properties is of no use for the reason which has just been considered in discussing the case of the conception of properties as grounds of objective resemblances.

${ }^{37} \mathrm{Cf}$. Owens (1989). A stronger version of the claim would then be that as long as one conceives of causal powers as causal explanantia, one can take disjunctions to be associated with new causal powers even in cases of non-disjunctive explananda.
} 
corresponding causal powers have been grounded, it follows that the property which grounds the causal powers of the disjunct in question can ground also the causal powers corresponding to the whole disjunction; there is no justification for introducing another property.

When properties are conceived of by reference to laws and theories, ${ }^{38}$ one can claim with some confidence that conclusions concerning disjunctions of properties are not going to be very different from the ones just considered. For one can regard theories as sets of laws; and laws and causation can obviously be regarded as unextricably intertwined. ${ }^{39}$

The case of the conception of properties as entities which are quantified over in laws is, then, particularly interesting insofar as it is perspicuous to bring out some features of the denial that dealing with subvenient properties can help to regard disjunctions of properties as themselves properties. I'll thus say a few words about that before concluding by gathering up the threads of what has been said so far.

When properties are conceived of as entities which are quantified over in laws, one can argue that not all disjunctions of properties are themselves properties either, by analogy with cases above, by taking the conception in

\footnotetext{
${ }^{38}$ See, e.g., Mellor (1991).

${ }^{39}$ Note, however, that the laws/causation nexus is not required for arguing against the possibility of taking all disjunctions of subvenient properties to be themselves properties. For - as we are
} 
question as specifying a property-role which is then argued to be absent in the case of at least some disjunctions, or, more generally, by taking the conception in question as specifying features which are to be satisfied for being properties, and which are then argued to be unsatisfiable by certain disjunctions. So, for example, one can argue that projectibility is a necessary condition for being a constituent of a law. ${ }^{40}$ It would then be possible to argue for the claim that not all disjunctions of subvenient properties are themselves properties by arguing, first, that not all disjunctions of properties are projectible, ${ }^{41}$ and, then, that the mere fact that what we are dealing with are disjunctions of subvenient properties can be of no help to ensure that the disjunctions in question are not among the ones which are not projectible.

The projectibility case is interesting in our context to the extent that it helps to bring out why the mere fact that what we are dealing with are subvenient properties can't be of any use to ensure that the disjunctions in question are not among the ones which are not properties. For projectibility is, typically, analysed

going to see below - one can argue against such possibility from considerations which do not rest on assuming the nexus.

${ }^{40}$ See, e.g., Goodman (1955).

${ }^{41}$ See Owens (1989). Owens further suggests that - to put it in our terms - some disjunctions can fail to be entities which are quantified over in laws to the extent that they fail to be explanatory of the consequent of the generalizations in which they figure. Note, however, that this can be true only under a notion of explanation which is not couched exclusively in terms of derivability/predictability. 
in terms of features such as entrenchment. ${ }^{42}$ And what this suggests is that supervenience fails to ensure the satisfaction of the feature required for being a property because of its very generality, because it says nothing of the kind about which one needs to know to establish whether the disjunctions in question are characterized by features such as, say, projectibility; property-supervenience does not provide much information about the properties which are subvenient and those which are supervenient.

When we consider forms of supervenience having as relata entities other than properties, we might, indeed, be provided with some information about the properties which feature in relations of supervenience which are entailed by the forms in question. So, for example, the supervenient and the subvenient properties of the relations of property supervenience which are entailed by event supervenience must have the features which are had by the properties which are constitutive properties of events. (Cf. $\S 1.5$.) But what we are not thereby told is then whether the disjunctions entailed by supervenient properties can, equally, be taken to possess the features in question. Even when we don't restrict our attention to property supervenience, no progress can thus be made against the claim that disjunctions of properties which are themselves properties cannot be obtained by supervenience alone.

All of that might look pretty dim for the prospect of ensuring property

\footnotetext{
${ }^{42}$ Goodman (1955). For the notion of 'entrenchment', see pp. 95 ff. What Goodman means by
} 
identity on the basis of supervenience alone. For what has been argued so far is, briefly, that (a) for a property to be identical to a determinate entity, this latter must itself be a property, and, hence, both predicates of the predicatecoextension required by the identity, must pick out properties; $(b)$ when properties are conceived of as truth-makers, or as grounds of objective resemblances, or as grounds of causal powers, or by reference to laws or theories, the holding of supervenience cannot guarantee that we'll have predicate-coextensions whose predicates pick out properties.

That, however, should not make us forget that what has been argued in this section is also that $(c)$ under a conception of properties as sets of particulars, supervenience can yield property identity. (And, in the psychophysical case, the identity yielded relates mental properties to physical properties if the notion of 'physical property' in play is one according to which a property can count as physical as long as it is instantiated exclusively by particulars possessing properties which are used in the physical sciences.) Moreover, $(d)$ if the notion of being nothing over and abovelontological reduction is understood, as in C1 and $\mathrm{C} 2$, in terms of entailment rather than identity, the holding of psychophysical supervenience can guarantee that mental properties are nothing over and above/reducible to physical properties under any conception of properties.

that is, roughly, the degree of use of the predicate in question in past projections. 


\subsection{Implications}

One of the claims of the Sceptic is that supervenience is consistent with most of the available options with regard to the Mind-Body problem. That, the Sceptic argues, speaks for the inadequacy of supervenience as a Mind-Body theory. ${ }^{43}$

What has been argued in this chapter, however, should help us to see that by making a supervenience claim one can rule out at least some positions with respect to the Mind-Body problem. In particular, a supervenience claim can rule out 'non-reductive' positions by being 'reductive'.

In this section I want to illustrate this point by considering first a nonreductive position par excellence, i.e. Davidson's Anomalous Monism, and, then, a position (i.e. Psychophysical Emergentism) that is regarded by some $\mathrm{e}^{44}$ as amounting, in fact, to the core commitments of a non-reductive physicalist.

As far as Davidson's position is concerned, I'll be brief. Davidson's Anomalous Monism is characterized by the denial of psychophysical laws. Although Davidson generally puts that in terms of a denial of psychophysical generalizations of a biconditional form ${ }^{45}$, I think that his denial of psychophysical laws is in fact to be taken to apply also to psychophysical

\footnotetext{
${ }^{43}$ See Kim (1997a; 1998).

${ }^{44}$ See, e.g., Kim (1992); Crane (forthcoming).

${ }^{45}$ See, e.g., Davidson (1970a), p. 214-5, and, also, Davidson (1973), p. 249, p. 251-2, p. 256; Davidson (1993), p. 7.
} 
generalizations of a conditional form. For Davidson's reason for denying psychophysical laws lies in his taking the conditions of ascription of the mental to be essentially different from those of the physical. And if psychophysical laws of a conditional form held from the physical to the mental, the conditions of ascription of the mental would not be different from those of the physical. (Consider: $C$ (i.e. the satisfaction of some conditions of ascription for physical states) is evidence for $P$ (i.e. the holding of a determinate physical state); $P$ entails $M$ (i.e. the holding of a determinate mental state); hence, $C$ is evidence for $M$.) As we have seen in the previous sections, there are at least some forms of supervenience that yield psychophysical conditionals which would allow for the ascription of mental states on the basis of the satisfaction of conditions of ascription of physical states. To this extent, there are at least some forms of supervenience which are inconsistent with Davidson's Anomalous Monism.

Turning to Psychophysical Emergentism (i.e. to the idea that the mental is emergent, in the senses to be considered below, with respect to the physical), it will take a bit longer, compared to the case of Davidson's Anomalous Monism, to bring out the inconsistency with some forms of psychophysical supervenience. For the notion of 'emergence' can be understood in different ways. And only by considering these various understandings it will be possible to show that there are forms of supervenience which are inconsistent with Psychophysical Emergentism. Moreover, it will also be necessary to underline 
the fact that to have a case of inconsistency, one needs determinate forms of supervenience as opposed to others, and more or less of the metaphysical assumptions considered in this chapter, according to the understanding of 'emergence' in play. The importance of that will be clear as a result of the remarks following the last chapter.

But, to begin with, let us consider the idea of emergence as unpredictability. One way of understanding that, is in terms of the idea that the instantation of an emergent property 'could not have been predicted in advance' (i.e. in advance of the time when the property actually gets instantiated). ${ }^{46}$

Now, under this understanding of emergence psychophysical supervenience can not be inconsistent with the idea that the mental is emergent with respect to the physical. For supervenience, at least as normally understood, is a synchronic relation. As such, it can't rule out by itself something which, as in the case under discussion, involves a temporal gap. ${ }^{47}$

\footnotetext{
${ }^{46}$ Beckermann (1992), p. 15.

${ }^{47}$ This, of course, is not to say that the impossibility of predicting the instantiation of the property in question can't be ruled out by psychophysical supervenience when this latter is combined with some other assumption. When, for example, it is possible to predict the instantiation of a property on which the property in question supervenes, the impossibility of predicting the instantiation of this latter can - it seems possible to say - be ruled out on the basis of supervenience. And if this is the kind of predictability that the claim that the property in question could not have been predicted in advance is to be taken to deny, it would no longer be true that psychophysical supervenience can't be inconsistent with the claim under discussion. That, however, would be so only because 'predictability' would have been read in a way very close to the one which is considered next in the main text.
} 
When, however, the notion of emergence is couched in terms of nonpredictability, what it is generally meant is rather something which can, less misleadingly, be put in terms of non-derivability. The idea is that when all that we have is the assumption that a non-emergent property $G$ is instantiated by an individual $x$, it is not possible, for any emergent property $F$, to derive the conclusion that $F$ is instantiated by $x .{ }^{48}$ So, for example, if the property of feeling a sharp pain in one's arm is taken to be an emergent property, and the property of being characterized by a determinate neural configuration $N$ is taken to be a non-emergent property, then the claim is going to be that from the fact that one is characterized by the neural configuration $N$ it is not possible to deduce that one is feeling a sharp pain in one's arm. ${ }^{49}$

This is not to rule out the possibility of obtaining $F x$ from assuming that some individual different from $x$ (or, indeed, and trivially, $x$ itself) is both $F$ and $G$. The Emergentist, that is to say, does not deny, for example, that once it has been found that the property of feeling a sharp pain in one's arm is coinstantiated with the property of being characterized by the neural configuration $N$, it will be possible to conclude that an individual $x$ is feeling a sharp pain in its arm from the fact that $x$ is characterized by the neural configuration $N$. To this extent, the

48 What I call 'non-emergent properties' are meant to be non-emergent with respect to the emergent properties considered. It is to be left open whether they can be emergent with respect to other properties.

${ }^{49}$ Cf. Kim (1992). 
emergentist position is not inconsistent with supervenience in general. For supervenience in general can give us conditionals of form $(\forall x)(G x \rightarrow F x)$, and, thus, the possibility of deriving $F x$ from assuming $G x$, only if some individual is assumed to be $F$ and, for this reason, to be $G$ as well. What supervenience in general can't give us is what, according to the present picture, is inconsistent with the emergentist position - i.e. conditionals of form $(\forall x)(G x \rightarrow F x)$ without the prior assumption that some individual is $F$ and, for this reason, also $G$. (From now on, this kind of conditionals will be referred to as ' $\mathrm{Cs}$ '. $)^{50}$

Cs, however, are yielded by SSS forms, i.e. by forms of 'SupervenienceSatisfactorily-Supported'. (See $\S 2.1$.) For they are yielded by each of the Assumptions.

That this is so is - I think - clear enough in the case of $A 2$ and $A 3$. (If identities hold between mental and physical properties, we can, for mental properties $M$ and physical properties $P$, have conditionals of form $(\forall x)(P x \rightarrow$ $M x$ ) without needing to assume that some individual has $M$ and, for this reason, also $P$. As for $A 3, \mathrm{Cs}$ are yielded in virtue of the fact that mental properties are assumed to stand to physical properties in a higher order/lower order relation, for

\footnotetext{
${ }^{50}$ I am here departing slightly from the picture given in Kim (1992) in that, according to this latter, the emergentist position is not actually inconsistent with Cs if these are 'brute facts'/inexplicable. But even if we add this further condition for inconsistency, the situation remains more or less the same: If supervenience in general cannot give us $\mathrm{Cs}$, then, a fortiori, it cannot give us instances of Cs which are not 'brute facts'. On the other hand, SSS forms do seem to yield, as we are going to see, instances of Cs which are not 'brute facts'.
} 
this latter is indeed a C.) In the case of $A 1$, by contrast, we need to provide a bit of an argument, and that can be done by recalling the steps of the reasoning developed in $\S 2.2$ to support the idea that MPS can be derived from $A 1$.

Among these steps, featured the assumption that a whole is nothing over and above the mereological sum of its parts. Such an assumption can, arguably, be taken as a claim about the properties of the wholes and mereological sums in question. In particular, then, the assumption could be understood as the claim that the properties of a whole are nothing over and above the properties of the corresponding mereological sums. As we have seen in $\S 3.3$, the notion of being nothing over and above for properties can be analysed either in terms of identity or in terms of entailments which, are, indeed, instances of Cs. So, either way, $A 1$ yields Cs. This, together with the fact that also $A 2$ and $A 3$ yield Cs, means that Cs are yielded by SSS forms. Given the assumed inconsistency between Cs and the emergentist position, this latter will thus be inconsistent with SSS forms although, as it will be remembered, it is not with supervenience in general. (We thus have a case where supervenience claims are made stronger as a result of (Th).)

Turn now to the idea of emergence as novelty. Once more there are different possible meanings in play. One idea is that an emergent property is a property which 'has never been instantiated before' (i.e. before the time the property is 
instantiated and judged emergent). ${ }^{51}$ Under this understanding of the idea of emergence as novelty, psychophysical supervenience can't - it may be argued be inconsistent with the claim that mental properties are emergent with respect to physical properties. For how could psychophysical supervenience by itself i.e. without the further assumption that a certain physical property has been instantiated - ensure that a particular mental property has been instantiated before? But the emergentist's claim can rather be read as requiring that an emergent property has not been instantiated before in individuals of a different type from the one in which it is found to be instantiated. Would it then, under this understanding of the idea of emergence as novelty, be, once again, impossible for psychophysical supervenience to be inconsistent with the claim that mental properties are emergent with respect to physical properties?

That depends on how the notion of type in the emergentist's claim is to be understood. If it is understood in such a way as to make sameness of type satisfiable only by individuals who share their (atomic) non-emergent properties, it does become impossible - it seems - for psychophysical supervenience to be inconsistent with the claim that mental properties are emergent with respect to physical properties. For to understand the notion of type in play in the emergentist's claim in such a way as to make sameness of type satisfiable only by individuals who share their (atomic) non-emergent properties, amounts to

\footnotetext{
${ }^{51}$ Beckermann (1992), p. 15.
} 
regarding the emergentist as in fact requiring that prior cases of instantiations of emergent properties be cases where the emergent property in question has been instantiated in an individual who is indistinguishable in its (atomic) nonemergent properties from the one in which the emergent property is instantiated at present. In other words, the emergentist would be regarded as requiring the holding of conditionals from individual emergent properties to individual atomic non-emergent properties; what she would be denying would, in the psychophysical case, be the existence of cases where we have the same mental property when we have different atomic physical properties. Psychophysical supervenience is, of course, consistent with such cases. On the other hand, however, it is also consistent with the possibility that all cases of instantiations of mental properties are not, actually, cases of this kind. To this extent, psychophysical supervenience isn't inconsistent with the possibility that the emergentist requirement under discussion can be satisfied. The impossibility of inconsistency between psychophysical supervenience and the claim that mental properties are emergent with respect to physical properties would thus follow from the impossibility of ensuring, on the basis of supervenience alone, that what the emergentist requires can't be the case.

But that the emergentist does require the holding of conditionals from individual emergent properties to individual atomic non-emergent properties is actually quite controversial. (Cf. Kim (1992).) Maybe the notion of type which is 
in play in the emergentist's claim is to be relaxed so as to allow for counting as individuals of the same type also individuals who share only disjunctions of nonemergent properties. If so, it would no longer be the case that it is impossible for psychophysical supervenience to be inconsistent with the claim that mental properties are emergent with respect to physical properties. For it would no longer be the case that the emergentist can be regarded as requiring something whose denial can't be ensured on the basis of supervenience alone.

When, then, one turns to other possible ways of conceiving of the idea of emergence as novelty, it can even be argued that psychophysical supervenience can, indeed, be inconsistent with the idea that mental properties are emergent with respect to physical properties.

To see that, consider, as an example, the idea that emergent properties are 'novel' with respect to the non-emergent properties in the sense that they are different from these latter. ${ }^{52}$ Under this conception of 'novelty', psychophysical supervenience can be inconsistent with the idea that mental properties are 'novel' (and, hence, emergent, under the idea of emergence as novelty) with respect to physical properties, to the extent that supervenience can ensure property identity. If what has been argued in $\S 3.3$ is true, supervenience can, under the conception of properties as sets of particulars, ensure property identity. Hence, psychophysical supervenience can be inconsistent with psychophysical 
emergence under the conception of emergence which is being considered.

From the fact that supervenience can - under the conception of properties as sets of particulars - ensure property identity, it then further follows that psychophysical supervenience can also ensure that mental properties are not emergent in the sense of being characterized by new causal powers. For if mental properties are identical to physical properties, it follows - by Leibniz's Law - that mental properties cannot be characterized by any causal power which cannot, also, be ascribed to physical properties. Note, however, that this entails that psychophysical supervenience is inconsistent with the idea that mental properties are emergent in the sense of being characterized by new causal powers, only if the conception of properties as sets of particulars is assumed. For - as we have seen in $\S 3.3$ - there are reasons for believing that unless the conception of properties as sets of particulars is assumed, supervenience cannot ensure property identity.

${ }^{52}$ See, e.g., Alexander (1920), p. 45, and what is claimed in Stephan (1992), p. 31, and Kim (1992), p. 123. 


\section{Dependence}

\subsection{The Desiderata}

As I mentioned in the Introduction, part of the original interest in the notion of supervenience was due to the hope that it could capture and provide a perspicuous formulation of the idea that the mental depends on the physical, an idea regarded as at least one of the core notions of a physicalist position. It was then characteristic of what I called 'the Sceptic's position' to pour cold water on this hope: The Sceptics argue that psychophysical supervenience alone cannot express psychophysical dependence.

This claim, however, is never made by reference to a definition of psychophysical dependence, on the basis of which supervenience could then be argued to be inadequate. Rather, what we can find in the literature is a somewhat mixed bag of conditions sometimes argued, but sometimes just assumed, to be necessary for having dependence. The idea is then that psychophysical supervenience is inadequate to express psychophysical dependence to the extent that it fails to ensure the satisfaction of all these conditions.

This is compatible with taking psychophysical supervenience to be necessary for psychophysical dependence. Indeed, it might well be (and, given the Sceptic's desiderata which will be considered, it probably is the case) that even 
the Sceptic would agree with taking something which is at least very close to supervenience to lie at the heart of a somehow intuitive idea of dependence. For $A$ to be dependent on $B /$ for $A$ not to be independent from $B$, it must be the case it seems - that there are no variations in $A$ unless there are variations also in $B .{ }^{1}$ If there are variations in $A$ without variations in $B$, then $A$ is independent from $B$. But then, the Sceptic's claim should probably be put as the idea that there are further requirements associated with 'dependence', which could not be satisfied on the basis of supervenience alone.

In what follows, I'll focus on these requirements (which I'll call the 'Desiderata'). In § 4.2 I'll resist the Sceptic's contention that the Desiderata are problematic for the idea that psychophysical supervenience can entail psychophysical dependence. I'll show that there are forms of supervenience which can ensure the satisfaction of the Desiderata at issue in the section, and/or that it can be questioned that this satisfaction is necessary for dependence. In $\S$ 4.3 , on the other hand, I will concede that some Desiderata are problematic (or at least more problematic than the ones considered in $\S 4.2$ ). This is because (i) there are no forms of supervenience which can ensure their satisfaction; (ii) there are better reasons, compared to the ones given for the Desiderata considered in $\S$ 4.2, for taking their satisfaction to be necessary for dependence; (iii) there are reasons for remaining unconvinced by attempts, considered in $\S 4.4$ and $\S 4.5$, at

\footnotetext{
${ }^{1}$ This is not quite supervenience because the covariance is not characterized by any kind of
} 
showing that it can't be that their satisfaction is necessary for dependence. As I'll argue in $\S 4.6$, however, these facts can't uncontroversially be used to support the Sceptical idea that supervenience is inadequate to express physicalist positions. So, in the end, even the 'problematic requirements' are not too problematic if the issue of whether supervenience can entail dependence derives its interest from the related issue of using supervenience to express physicalist positions.

\subsection{The less problematic : modal force and the wayward}

\section{atom}

In considering alleged inadequacies of weak supervenience, Jaegwon Kim claims that 'Determination or dependence is naturally thought of as carrying a certain modal force [...]'.2 A similar claim reappears in Kim (1990a) (although this time the claim is qualified by the presence of an 'arguably'): '[...] modal force is arguably a necessary aspect of any significant dependency claim. ${ }^{3}$

necessity.

${ }^{2} \operatorname{Kim}(1984 a)$, p. 60.

${ }^{3} \operatorname{Kim}(1990 \mathrm{a})$, p. 143. 
The kind of 'modal force' Kim has in mind is the one that, if required for the dependence of, say, $A s$ on $B s$, would bar any cross-world difference in As which is not accompanied by a cross-world difference in Bs. The idea seems to be that the lack of this kind of co-variance would clearly be inconsistent with the dependence of the $A s$ on the $B s .{ }^{4}$

But is it so? The problem is that, as I have mentioned in the previous section, those, like Kim, who blame claims of supervenience for not satisfying certain conditions, never give a definition of 'dependence' - let alone an uncontroversial one. In order to establish whether the conditions in question should be taken as requirements for dependence, we are thus left with an appeal to intuitions - to what people would say about the conditions in question and about what the absence of their satisfaction would allow for. But then, it is not clear that there are clear-cut intuitions which go one way or the other. In the present case, for instance, there are people, such as William Seager ${ }^{5}$, who would deny that the kind of modal force Kim has in mind is necessary for dependence. Seager, in particular, is then quite explicit about his regarding the lack of crossworld co-variance between, say, $A s$ and $B s$, as perfectly consistent with the dependence of $A s$ on $B s .{ }^{6}$ Moreover, the disagreement here (with those who take Kim's modal force to be necessary for dependence) is not just terminological,

\footnotetext{
${ }^{4}$ See, again, Kim (1990a), p. 143.

${ }^{5}$ See Seager (1988).

${ }^{6}$ See esp. p. 709.
} 
but it seems to be quite substantial. For the two parties are not merely disagreeing on which, between two completely different notions, should be given the name of 'dependence'. The notion they are discussing is, on the contrary, the same to the extent that in the psychophysical case it is meant to do the same job of expressing one of the desiderata of physicalism. The issue of whether Kim's modal force is necessary for dependence is, therefore, a real one. But, given that, as I said and as the case I mentioned shows, there are no clearcut intuitions one way or the other, it is also an issue that, to a certain extent, is to be left open.

Even if, anyway, Kim's modal force were necessary for dependence, it would still not follow that we would have a problem for the idea that psychophysical supervenience can entail psychophysical dependence. For, although weak supervenience does fail to have Kim's modal force, the same can't be said of NRSS and of global supervenience. In other words, there are forms of supervenience which fulfil the requirement under discussion.

Another case which is supposed to be problematic for just one kind of supervenience, is that of 'the wayward atom'. Once again, it comes from Kim:

'[...] it is consistent with this version of materialism [i.e. a version of materialism which is formulated in terms of the global supervenience of mental properties on physical properties] for there to be a world which differs physically from this world in some most trifling respect (say, Saturn's rings in that world contain one more ammonia molecule) but which is entirely 
devoid of consciousness, or has a radically different, perhaps totally irregular, distribution of mental characteristics over its inhabitants (say, creatures with brains have no mentality while rocks are conscious) ${ }^{[7]}$ As long as that world differs from this one in some physical respect, however minuscule or seemingly irrelevant, it could be as different as you please in any psychological respect you choose. [...] It is doubtful that many materialists would regard these consequences as compatible with their materialist tenets; it seems clear that they are not compatible with the claim that the mental is determined wholly by the physical. ${ }^{8}$

The suggestion is, thus, that the global supervenience of mental properties on physical properties is consistent with the independence of the mental and the physical; hence, the global supervenience of mental properties on physical properties cannot ensure the dependence of the mental on the physical. ${ }^{9}$

But why is the case envisaged supposed to be inconsistent with the dependence of the mental on the physical? What is it that makes the case

\footnotetext{
${ }^{7}$ In Kim (1989a) the same kind of situation is put in terms of the world in question differing from the actual world in that 'one lone hydrogen atom somewhere in deep space is slightly displaced relative to its position in this world' (Kim (1989a), p. 277) - hence the association, in the literature, of the term 'wayward atom' with this kind of example. Although I follow the literature in using the term 'wayward atom' when speaking of this kind of example, I have preferred to quote the (1987) version instead of the (1989a) version because I think that the (1987) version is more suitable to bring out what I want to consider.

${ }^{8}$ Kim (1987), p. 85-6. (Italics mine.)

${ }^{9} \mathrm{I}$ am here following common practice (and, indeed, Kim's practice too) in taking dependence as the converse of determination, so that if $A$ determines $B$ (if the physical determines the mental; if - as in the claim mentioned in Kim - 'the mental is determined by the physical'), $B$ is dependent on $A$ (the mental is dependent on the physical). Objections to this use will be considered later.
} 
envisaged inconsistent with the dependence of the mental on the physical?

Kim's complaint about the physical difference between the two worlds being 'minuscule or seemingly irrelevant' provides a clue to answer these questions. The suggestion seems to be that the case envisaged is inconsistent with the dependence of the mental on the physical to the extent that, given a relation of co-variance between mental and physical properties, the physical differences which are entailed by mental differences are 'minuscule or seemingly irrelevant'. So, putting things the other way round, we can say that the idea is that the dependence of the mental on the physical requires that, given a relation of co-variance between mental and physical properties, the physical differences which are entailed by large mental differences, be themselves large differences, and, furthermore, differences which are relevant to the mental differences in question.

Here are, then, two desiderata for psychophysical dependence:

(WA1) $\left[=1^{\text {st }}\right.$ Wayward-Atom-Desideratum $]$ Given a relation of co-variance between mental and physical properties, the physical differences which are entailed by large mental differences must themselves be large.

(WA2) $\left[=2^{\text {nd }}\right.$ Wayward-Atom-Desideratum $]$ Given a relation of co-variance between mental and physical properties, the physical differences which are 
entailed by mental differences must be differences which are relevant to the mental differences.

Now consider (WA1). Is strong supervenience or weak supervenience better suited than global supervenience to ensure its satisfaction? It does not seem so. Strong supervenience and weak supervenience too are consistent with cases where large differences in mental properties are accompanied by only small differences in physical properties. (The mental properties of two individuals can, consistently with strong supervenience and weak supervenience, differ a lot as long as their physical properties differ a bit - e.g. in the activation of a few neurons of their brains.) So, one point to be made about (WA1) is that it can't actually help to see why the case of the wayward atom should be a problem only for global supervenience. ${ }^{10}$

Another point is that it is not actually clear why we should take (WA1) as a requirement for having psychophysical dependence. For one thing, there seem to be cases where we do make claims of psychophysical dependence even if we have only small physical differences to couple with large mental differences. ${ }^{11}$

\footnotetext{
${ }^{10}$ Cf. Paull \& Sider (1992).

${ }^{11}$ The same point is made in Post (1995). Post, however, supports this claim by appealing to cases where there is a temporal gap between the physical difference and the mental difference. (He appeals, for example, to dependence claims involving physical differences at or just after the Big Bang and differences in the present state of the world.) Maybe this feature of Post's examples has, actually, no importance in our context. For, it could be argued, whether or not the
} 
Think, for example, of the mental disorders which are claimed to be dependent on small tumors in the temporal lobe. Moreover, even if there weren't such cases, one could still argue against taking (WA1) as a necessary requirement for having dependence on the grounds that when we do make claims of dependence, a change in the situation which is restricted to the mere size of the physical and mental differences in question wouldn't - it seems - necessarily lead us to withdraw such claims of dependence. (Suppose, for example, that we are willing to regard having a certain memory experience as dependent on the activation of a certain number of neurons in one's brain. Would we be withdrawing any claim of dependence in case it turned out that differences with respect to the having of the memory experience in question are actually correlated with differences in fewer neurons than the ones with respect to which the original dependence claim was made?)

As for (WA2), one can wonder what it means, in fact, to require that the physical differences entailed by mental differences be relevant to the mental differences. Does it mean that the physical differences entailed by the mental differences must be relevant to the determination of the mental differences? If so, it would, of course, be trivially true that what satisfies (WA2) can ensure

size of the physical and mental differences in question matters for dependence does not seem to have anything to do with whether there is a temporal gap between the physical difference and the mental difference in question. Still, I have preferred to make use of an example where there is no 
dependence. But then, to argue for the failure of psychophysical supervenience to entail psychophysical dependence on the grounds of an alleged failure of supervenience to satisfy (WA2) would amount to assuming that psychophysical supervenience can't entail psychophysical dependence.

Perhaps, though, what Kim's claim about relevance can be taken to suggest is actually another alleged kind of deficiency of global supervenience with respect to ensuring psychophysical dependence; a deficiency that can be brought out by reflection on the case of the wayward atom. The idea is that global supervenience would be deficient with respect to ensuring psychophysical dependence to the extent that claims of global supervenience would be 'unfocused' - i.e. they would, in the psychophysical case, concern the whole sets of the mental and of the physical properties. By contrast, what we have in mind, when we say that the mental depends on the physical, are relations holding between sets of mental properties and those physical properties which are relevant to the determination of the mental properties in question. ${ }^{12}$ If we want to put that in terms of a desideratum for having psychophysical dependence, we can say the following:

temporal gap between the physical difference and the mental difference in question - as in discussing supervenience, we are normally dealing with simultaneous co-variations.

${ }^{12}$ The suggestion that this is something that reflection on the case of the wayward atom can be taken to bring out, is made in Post (1995). 
(WA3) [ $3^{\text {rd }}$ Wayward-Atom-Desideratum $]$ To have a relation of psychophysical dependence, we must have, as relata, specific sets of mental and physical properties; the whole families won't do.

Once again it is not clear that strong supervenience or weak supervenience are better suited than global supervenience to satisfy the requirement under discussion. For the relata of a relation of supervenience can be whole families of properties even if the distribution bases are constituted by single individuals as opposed to entire possible worlds.

The main question concerning (WA3), however, is - similarly to what was the case for (WA1) - that of why one should take that as a requirement for having psychophysical dependence. ${ }^{13}$ It might well be that whenever we have psychophysical dependence, there are sets of mental properties which are related to sets of physical properties which are relevant to their determination. What is not clear, however, is that we can speak of dependence only when the properties we are concerned with are the ones which stand in such relations. Even if whenever the mental depends on the physical there are sets of mental properties which are related to sets of physical properties which are relevant to the determination of the mental properties in question, it does not follow that what we have in mind, when we say that the mental depends on the physical, are these sets of properties, and not the whole sets of the mental and of the physical properties. (More or less the same point - I take it - is made in Post (1995). Post

\footnotetext{
${ }^{13}$ Here again, not having a definition, the grounds for answering this question are going to be what people are, or are not, willing to regard as dependent. And as I suggest below, intuitions do not, in the present case as before, seem to be clear-cut.
} 
supports the point by likening the psychophysical case to that of the relation between the theorems and the axioms of an axiom system. In such a case - he argues - there might well be particular sets of theorems which are related to particular sets of axioms which are relevant to their determination. From that, however, it does not follow that we can speak of the dependence of theorems on axioms only when the axioms we are talking about are restricted to those which are relevant to the determination of the theorems which we are considering. ${ }^{14}$ )

In case, however, one were not convinced by that, and, thus, did want to hold onto (WA3) as a requirement for having psychophysical dependence, what would not be clear would be that one would thereby be committed to denying that psychophysical supervenience can entail psychophysical dependence. For the satisfaction of (WA3) can be ensured on the basis of psychophysical supervenience. The term 'psychophysical supervenience' need not refer only to relations between the whole set of the mental properties and the whole set of the physical properties. It then seems to me that it would be question begging to object that what cannot be ensured is that by making claims of psychophysical supervenience, the physical properties we are concerned with are those which are relevant to the determination of the mental properties we are concerned with. No further reason would have been given for saying that. It is not as if we had been provided with a new requirement which - it can be shown - cannot be

\footnotetext{
${ }^{14}$ Note that the very fact that Post takes the position described supports my claim that there are no clear-cut intuitions here. (Post can be taken as an instance of someone who denies that we can speak of psychophysical dependence only when we are dealing with specific sets of mental and physical properties, as opposed to the whole families.) And again, the disagreement here is not merely terminological, in view of the role that the notion of dependence is supposed to play in delineating the concept of physicalism.
} 
fulfilled in virtue of psychophysical supervenience alone.

If (WA3) can be satisfied on the basis of psychophysical supervenience alone, we'll have one more reason for taking (WA3) as not problematic for the idea that psychophysical supervenience can entail psychophysical dependence. (The other reason is that - as we have seen - it is debatable whether (WA3) should be taken as a requirement for having psychophysical dependence.) As for (WA1), the situation is different to the extent that its satisfaction is not something that can be ensured by psychophysical supervenience alone. (From the fact that the mental supervenes on the physical it does not follow that, given a relation of covariance between mental and physical properties, the physical differences which are entailed by large mental differences are themselves large.) On the other hand, though, it is also true that - as in the case of (WA3) - doubts can be raised about the idea that (WA1) is a plausible requirement for having psychophysical dependence. Finally, I have argued that it would be questionbegging to argue for the failure of psychophysical supervenience to entail psychophysical dependence on the grounds of an alleged failure of supervenience to satisfy (WA2). So, it would seem that, all in all, also (WA1) and (WA2) are not too problematic for the idea that psychophysical supervenience can entail psychophysical dependence. If that is true, it follows under the assumption that (WA1), (WA2), and (WA3) do exhaust all the desiderata which can be extracted from the case of the wayward atom - that it is actually the whole of the wayward atom case which is not too problematic for the idea that psychophysical supervenience can entail psychophysical dependence. And the same conclusion should obviously apply to the requirement of modal force, since - as we have seen - it is for one thing questionable that 
Kim's modal force should be taken as a requirement for having dependence; moreover, even if Kim's modal force were taken as a requirement for having dependence, there are forms of supervenience which could ensure its satisfaction. In the next section, by contrast, we'll be considering cases which seem to be more problematic.

\subsection{The more problematic: asymmetry and explanation}

Dependence is frequently assumed to require asymmetry. ${ }^{15}$ The relation of dependence - it is maintained - is such that if $A$ depends on $B$, then $B$ does not depend on $A$. If so, psychophysical supervenience cannot by itself entail psychophysical dependence. For, as it has been pointed out by many ${ }^{16}$, supervenience is neither symmetric nor asymmetric. From the supervenience of $A$ on $B$, it thus cannot be concluded that $A$ is asymmetrically related to $B$, as it should be if $A$ is dependent on $B$, and if dependence requires asymmetry.

But why should dependence be taken to require asymmetry? Indeed, how can that be if - as it seems to be the case - it makes perfectly good sense, in some cases, to speak of 'mutual dependence'? (Think, for example, of three points, A, $\mathrm{B}$, and $\mathrm{C}$, standing on a line in the following order: $\mathrm{A}$ is followed by $\mathrm{B}$, and $\mathrm{B}$ is followed by $\mathrm{C}$. In such a case, one might want to say that the distance of $\mathrm{B}$ from

${ }^{15}$ See, e.g., DePaul (1987); Petrie (1987); Kim (1990a; 1997a; 1998); Savellos \& Yalçin (1995b).

${ }^{16}$ See, again, DePaul (1987); Kim (1990a; 1997a; 1998); Savellos \& Yalçin (1995b); but, also, e.g., Macdonald (1995). 
C depends on the distance of B from A, but also that the distance of B from A depends on the distance of B from C.)

I will now consider what can be said in support of the idea that dependence requires asymmetry. If the argument works, the idea that it makes perfectly good sense, in some cases, to speak of 'mutual dependence' can then be accommodated by distinguishing between types of dependence: that which is symmetric and that which is asymmetric. As we are going to see in $\S 4.6$, it will then be possible to ask, when considering the dependence requirement within the context of issues such as that of the relation between physicalism and supervenience, which of the two types of dependence is the one that is in play.

The idea that dependence requires asymmetry can be supported on the basis of an argument of the following form:

(i) Dependence entails explanation - for $A$ to depend on $B, A$ must be explainable in terms of $B$.

(ii) Explanation is asymmetric.

$\therefore$ (iii) Dependence is asymmetric.

Premiss (i) can itself be taken as one of the Desiderata. ${ }^{17}$ And, given that - as we have seen - supervenience cannot ensure asymmetry, (i) is, in the light of (ii), going to be problematic for the idea that psychophysical supervenience can

\footnotetext{
${ }^{17}$ See Kim (1990a); Bonevac (1995); Moser \& Trout (1995).
} 
entail psychophysical dependence. On the other hand, however, (i) and (ii) are both questionable. In particular, it can be questioned that there are good reasons for endorsing them. In response to that, I'll now consider what can be said in support of (i). As for (ii), a satisfactory assessment would require one to sail into the deep waters of an analysis of the notion of 'explanation'. I am thus compelled to leave the issue open here, and to rest content with pointing out that at least for those who take explanation to be asymmetric ${ }^{18}$, there is going to be a problem with the idea that supervenience can entail dependence, if dependence is assumed to entail explanation.

Some kind of support for the claim that dependence entails explanation might be obtained from the following case. ${ }^{19}$ Suppose that both one's degree of manual dexterity (DMD) and one's degree of intelligence (DI) depend on certain genetic and developmental factors (GDF), and that DI supervenes on DMD. In such a situation - it can be argued - we wouldn't say that DI is dependent on DMD. But, what is it that might account for our willingness to allow for the possibility that DMD and DI depend on GDF, as opposed to our denying that something like DI can depend on something like DMD? What is the difference between such things as DMD and DI with regard to such things as GDF, and such things as DI with regard to such things as DMD, that can account for our willingness to allow for the possibility that DMD and DI depend on GDF, as opposed to our denying that something like DI can depend on something like DMD? Well, it is at this point that the appeal to explanation might gain some plausibility. The

\footnotetext{
${ }^{18}$ See, e.g., Hempel (1965); Nozick (1981); Salmon (1998).

${ }^{19}$ The example is taken from Kim (1990a), although it is here used, somehow differently, to make a different point.
} 
reason - it can be argued - why we are willing to allow for the possibility that DMD and DI depend on GDF, whereas we feel inclined to deny that something like DI can depend on something like DMD, is that such things as DMD and DI seem to be the sort of things which can be explained on the basis of things such as GDF, whereas something like DI does not seem to be explainable in terms of DMD. But then, that suggests that the notion of dependence is closely related to that of explanation. And the reason why the dependence of DI on DMD is denied might lie in the fact that we are assuming that one's DI cannot be explained in terms of DMD; dependence would - according to such a picture entail explanation.

These considerations are not meant to be conclusive. For I think there is still room for disagreeing on the idea that what grounds our willingness to allow for the possibility of the dependence of DMD and DI on GDF, as opposed to our denying that something like DI can depend on something like DMD, is indeed the fact that such things as DMD and DI seem to be the sort of things which can be explained on the basis of things such as GDF, whereas something like DI does not seem to be explainable in terms of DMD.

What is shown by the considerations above, however, is at least that, contrary to what was the case for the Desiderata of the previous section, it is possible to have some kind of argument (though, maybe, still controversial) in support of the Desideratum in question - i.e., in the present case, the idea that dependence entails explanation. To this extent, the present Desideratum is more problematic than the Desiderata of the previous section, for the idea that psychophysical supervenience can entail psychophysical dependence. Moreover, in the light of the argument above, also the Desideratum of asymmetry is going to be 
problematic. For if it is possible to support the idea that dependence entails explanation, then, under the assumption that explanation is asymmetric, it will be possible to support also the idea that dependence is asymmetric; and, as we have seen, supervenience does not yield asymmetry. I now want to consider attempts at showing that it can't be that dependence entails asymmetry and explanation. (If these attempts were successful, one or more of the premisses in the arguments of this section would have to be rejected.)

\subsection{First attempt to rescue supervenience : versus asymmetry and explanation.}

In Post (forthcoming) it is argued that it can't be that dependence entails asymmetry and explanation. In case Post were right, it would follow that the impossibility of ensuring asymmetry and explanation on the basis of supervenience alone is not problematic for the idea that psychophysical supervenience can entail psychophysical dependence.

Post's argument to the conclusion that it can't be that dependence entails explanation can be put as follows.

(i) Dependence (or determination ${ }^{20}$ ) must - at least in the case which is of

\footnotetext{
${ }^{20}$ Throughout his paper, Post uses the terms 'determinational dependence' and 'determination'. As the remarks at p. 3 make clear, what he means by that is the converse of dependence. ( ' $[\ldots]$ let us follow Kim in using the word 'determination' to mean [...] a kind of dependence.') So, I'll here use the terms 'determination' and 'dependence' indifferently.
} 
interest to us - be transitive; if $A$ depends on $B$, and $B$ depends on $C$, then $A$ depends on $C$.

(ii) If dependence is transitive, the relation of explanation which is allegedly implied by dependence would have to be transitive too.

(iii) The kinds of explanation which are relevant to our case are not transitive.

$\therefore$ (iv) It can't be that there is a relation of explanation which is implied by dependence; it can't be that dependence entails explanation.

The first premiss is supported, in Post, by the following considerations. The relation of dependence which is of interest to us is that which the physicalist speaks about. ${ }^{21}$ And this latter is (and it needs to be) transitive. When the physicalist claims that the quantum-chemical properties depend on (or are determined by) the quantum-physical properties, for example, she also wants to claim that, since the biochemical properties are dependent on the quantumchemical properties, the quantum-physical properties determine also the biochemical properties. Moreover, determination must, for the physicalist, be transitive to the extent that this is necessary for supporting the physicalist claims

\footnotetext{
${ }^{21}$ This is in accordance with the general framework, outlined in the Introduction, of an interest in the notion of 'supervenience-as-dependence' motivated by the hope of capturing at least part of what lies at the heart of a physicalist position. As I have mentioned in the previous section, it might well be that there is more than one type of dependence. If so, our attention will, indeed, be focused, as in Post, on the type which is supposed to be relevant when considering physicalism.
} 
of determination. For it is unlikely that we can find some direct support for a claim of dependence on physical properties of kinds of properties such as the psychological properties. Rather, the support for this kind of claim is more likely to come, if at all, from supporting the dependence, on the physical properties, of kinds of properties on which, say, the psychological properties depend (or of kinds of properties which determine other kinds of properties on which the psychological properties depend, or... so on and so forth, adding as many steps as you want). But in order to support, say, psychophysical dependence in this way, one needs the relation of dependence to be transitive. It follows that, if we want to give the physicalist the chance of supporting her claims of dependence on the physical, we must take the relation of dependence she speaks about to be transitive. Thus, the relation of dependence which is of interest to us must be transitive, if it is the ome which the physicalist speaks about.

Premiss (ii) can then be shown to be acceptable by considering the following case. Suppose that a thing's biological property $\mathrm{N}$ is determined by the thing's biochemical properties $\mathrm{Bi}$, and that these latter are determined by certain quantum-chemical properties $\mathrm{Pi}$. Then, if determination is transitive, $\mathrm{N}$ will be determined by Pi. Now suppose that there is a relation of explanation which is implied by dependence ( dependence relation between $\mathrm{N}$ and $\mathrm{Bi}, \mathrm{Bi}$ and $\mathrm{Pi}, \mathrm{N}$ and $\mathrm{Pi}$, it would then follow that also $\mathrm{E}$ holds between $\mathrm{N}$ and $\mathrm{Bi}, \mathrm{Bi}$ and $\mathrm{Pi}$, and $\mathrm{N}$ and $\mathrm{Pi}$. Generalizing, a relation of explanation will follow from relations of explanation of the kind considered, whenever we have a case where a relation of dependence follows from relations of dependence of the kind considered. In other words, a relation of explanation which is implied by dependence is transitive if dependence is 
transitive.

What is more controversial is premiss (iii) - i.e. the claim that the kinds of explanations which are relevant to our case are not transitive. Post considers, for example, the relation which holds between two sets of properties when the instantiation of properties from one set is the best explanation for the instantiation of properties from the other. Such a relation (call it 'the relation of best explanation', or 'BE' for short) is not transitive. For from the fact that the instantiation of properties from a set $A$ is the best explanation of the instantiation of properties from a set $B$, and that the instantiation of properties from $B$ is the best explanation for the instantiation of properties from another set $C$, it does not (and, indeed, given that for any one explanandum there can be only one best explanation, it can not) follow that the instantiation of properties from $A$ is the best explanation of the instantiation of properties from $C$. Similarly for a kind of explanation characterized by the explanans's making the explanandum suffïciently probable - i.e. reaching the degree of probability (generally greater than 0.5 ) which is judged sufficient for having an explanation. (This kind of explanation will be called 'probabilifying explanation', or 'PE', for short.) $\mathrm{PE}$ is not transitive. For from the fact that A makes B 'sufficiently probable' (by, e.g., bringing B's probability to 0.7 when the degree of sufficient probability has been assumed to be 0.6 ), and that B makes $C$ sufficiently probable, it does not follow that A makes $\mathrm{C}$ sufficiently probable. (The degree of probability that A can ensure for $C$ will be less than that which $A$ can ensure for $B$ and than that which $\mathrm{B}$ can ensure for $\mathrm{C}$. For the degree of probability that $\mathrm{A}$ can ensure for $\mathrm{C}$ is given by the product of the degree of probability that A can ensure for B and the degree of probability that B can ensure for C. So, for example, if the degree of 
probability that A can ensure for B is 0.6 , and the degree of probability that $\mathrm{B}$ cam ensure for $\mathrm{C}$ is again 0.6 , then the degree of probability that $\mathrm{A}$ can ensure for $\mathrm{C}$ iis 0.36 . Our 'sufficient degree of probability' might then be 0.6.) Finally, no transitivity can be claimed either, for those explanations which require some specific interlevel theories. ${ }^{22}$ (This kind of explanation will be called 'inter-leveltheory-explanation', or 'ILTE', for short.) For from the existence of a relevant interlevel theory between $\mathrm{A}$ and $\mathrm{B}$, and between $\mathrm{B}$ and $\mathrm{C}$, it does not follow that a theory of the relevant kind will exist also between $\mathrm{A}$ and $\mathrm{C}$.

But all that this shows is that $\mathrm{E}$ cannot - given the transitivity requirement be a case of ILTE, or of PE, or of BE. What is not clear, however, is why one should take the kinds of explanations which are relevant to our case to be exthausted by ILTE, PE, and BE.

Im the example discussed when considering premiss (ii), the interlevel properties are, arguably, related to the bottom and top level properties by at least two of the non-transitive relations mentioned - i.e. BE and ILTE. One might thus think that the explanation relations which are relevant to our case are not transitive, if they are constituted by the explanation relations which involve interlevel properties ${ }^{23}$.

But from the fact that, say, $\mathrm{Bi}$ is related to, say, $\mathrm{Pi}$ and $\mathrm{N}$ by explanation relations which are not transitive, it does not follow that it can't, also, be related

\footnotetext{
${ }^{22}$ Siee Post (forthcoming), pp. 8 ff.

${ }^{23}$ The kinds of explanation relations which are relevant to our case are - one can argue conistituted by the explanation relations which involve interlevel properties, insofar as interlevel properties are among the relata we are concerned with. (This, in turn, is true because of what has been said in support of premiss (i).)
} 
to $\mathrm{Pi}$ and $\mathrm{N}$ by explanation relations which are transitive. For it is not clear why one should deny that something can stand in more than one relation with the same things.

To that it could be objected that what is not clear is actually what these other transitive relations could be. Moreover, the fact that these transitive relations cannot be cases of $\mathrm{BE}$, or of $\mathrm{PE}$, or of ILTE, could be regarded as problematic, insofar as - it could be argued - this fact would mean that all the fundamental kinds of explanation would, thereby, have been precluded as kinds of explanation to which E can belong.

What follows from the fact that these transitive relations cannot be cases of $\mathrm{BE}$, or of PE, or of ILTE, however, is not that BE, PE, and ILTE are, in our context, precluded from holding tout court. (As I've just pointed out, there does not seem to be any inconsistency in maintaining that something can stand in more than one relation to something else; thus, in the case considered, for example, there does not seem to be any inconsistency in allowing for there being a transitive relation of explanation between, say, $\mathrm{Bi}$ and $\mathrm{N}$, while, at the same time, still maintaining that $\mathrm{Bi}$ is related to $\mathrm{N}$ by, say, ILTE. ${ }^{24}$ ) Rather, what

\footnotetext{
${ }^{24}$ In the case of $\mathrm{BE}$, the situation is actually a bit trickier. For what is supposed to be an explanation when something is the best explanation of something else is the same as what is the best explanation. (So, for example, if the relata are $\mathrm{Bi}$ and $\mathrm{N}$, it will be $\mathrm{Bi}$ that is going to be both the best explanation and an explanation.) Correspondently - it might be argued - what is supposed to be a relation of explanation when something is the best explanation of something else is the same as the relation of best explanation. So, it is not clear how we can have both a transitive and a non-transitive relation of explanation here. (Since something cannot be both transitive and non-transitive.)

However, the quandary can be solved by appealing to the possibility of considering the explanation in question under certain features and to the exclusion of others. (In our case, Bi can
} 
follows from the fact that these transitive relations cannot be cases of BE, or of $\mathrm{PE}$, or of $\mathrm{ILTE}$, is that $\mathrm{BE}, \mathrm{PE}$, and ILTE are precluded as kinds of explanations to which E can belong. But then, what can be questioned is that what follows from the fact that these transitive relations cannot be cases of $\mathrm{BE}$, or of $\mathrm{PE}$, or of ILTE, is that all the fundamental kinds of explanations have, thereby, been precluded as kinds of explanation to which E can belong. So, for example, consider PE. If all kinds of probabilistic explanations were to be analysed in terms of PE, the preclusion of PE would, certainly, be problematic. But that all kinds of probabilistic explanations are to be analysed in terms of PE is, actually, quite controversial. Are analyses in terms of the explanans's raising the probability of the explanandum to be excluded? Or, indeed - in case one were not happy about this kind of analysis because one thinks that they too would lead to the nontransitivity of the relation of explanation in question - what about those notions of explanation according to which the explanandum is not required to stand in any specific degree of probability with respect to the explanans, but, rather, what is required is that this latter change (without, however, necessarily increasing) the degree of probability of the explanandum? $?^{25}$

be considered either under those features which make of it an explanation of $\mathrm{N}$, or under those additional? - features which make of it the best explanation of N.) Correspondently, the relation of explanation in question can be considered either as a relation of explanation in general (thus allowing - under this form - for transitivity) or as a relation of best explanation (and it is, of course, this feature of it - i.e that of being a relation of best explanation - which is not transitive). [Note that the point can be put also in terms of what is transitive or not transitive being the second-order properties of being a relation of explanation and of being a relation of best explanation.]

${ }^{25}$ Cf. Salmon (1970), pp. $62 \mathrm{ff}$. As an example of an explanation where the explanandum is made less probable by the explanans, Salmon puts forward the case of the tossing of a coin 
This kind of explanatiom can, besides, be used as an example to respond to the objection that it is mere hand-waving to appeal to the possibility of transitive relations between interleveel properties. And there is, then, the notion of explanation as derivabilitty//entailment considered in ch. 3 . It thus seems that there are no reasons for thinking that some of the explanations which are relevant to our case can'tt bse transitive. And no conclusive reasons have been given either for thinking that the kinds of explanations which are relevant to our case are not, in fact, transittive. It has thus not been possible to establish the conclusion of the argumemt, in which this idea appears as one of the premisses, to the effect that it can't be that dependence entails explanation.

Is the situation any bietter with regard to the claim that it can't be that dependence requires asymimeetry?

Post supports this claim ron the basis of the following considerations. There are cases - Post argues - where we might want to say that determination obtains 'by way of identity' (or, allternatively, by way of nomological equivalence ${ }^{26}$ ). So, for example, suppose thatt the temperature of a cup of tea is identical with the tea's mean molecular kineticc energy. In such a case it might be that we want to say not only that the temipeerature of the cup of tea is identical with the tea's mean molecular kinetic enierģgy, but also that, on the basis of this identity, we can say that the tea's temperature is determined by its mean molecular kinetic energy. Similarly, if the prosperty of being red is identical with the property of

biased for tails and of its landinig theads; the contention is that the tossing of the coin can explain its landing heads even if, being a ttossing of a coin biased for tails, it in fact decreased the chance of the coin landing heads.

${ }^{26}$ The possibility of running thee argument in terms of nomological equivalence instead of identity will have to be borne in miind. I won't keep on reminding the reader of that. 
having a certain triplet off electromagnetic reflectance efficiencies, we might want to say that the formeer is determined by the latter, on the basis of this identity. But if the properrty of being red is both identical to and determined by the property of havings a certain triplet of electromagnetic reflectance efficiencies, it will follow - by substitutivity of identicals - that it will also be the case that, converscelyy, the property of having a certain triplet of electromagnetic reflectancces efficiencies is determined by the property of being red. Generalizing, we can thus say that there can be cases where (1) a property $A$ is identical with a property $a B$; (2) on the basis of this identity, we can say that $A$ determines $B$; (3) by substtitutivity of identicals, $B$ determines $A$. That shows that it is not true that the relation of determination is asymmetric. (Since for the relation of determination 1to be asymmetric, it must be true that if $A$ determines $B$, then $B$ does not determiince $A$.)

My worry with this arrgument is that it seems to presuppose, after all, that determination does not enttaiil explanation (or at least explanation conceived of in a certain way, i.e. as ruliing out that things can be self-explanatory, and that nomological equivalents ccain explain each other). For Post's assumption that in at least some cases of ideenttity (or nomological equivalence) we would still be willing to speak of determiination is, in fact, hardly uncontroversial. And, indeed, only if one has already rejjected the idea that determination entails explanation, one can - it seems - be willling to speak of determination in cases of identity or nomological equivalence.. For suppose that, on the contrary, one believes that determination does entail explanation, and, furthermore, one also believes that things can't be self-explannatory and that nomological equivalents can't explain each other. Then, one woulddn't be willing to say that $x$ determines $y$ when $x=y$ 
(or when $x$ and $y$ are nomological equivalent). If that is true, it follows that Post's argument to the effect that it can't be that determination entails asymmetry is, after all, dependent on his claim that it can't be that determination requires explanation. ${ }^{27}$ And if - as it has been suggested - this latter claim has not been conclusively supported, the claim that it cannot be that determination requires asymmetry cannot, thus, be conclusively supported either. That means that the attempt to rescue the idea that psychophysical supervenience can entail psychophysical dependence by appealing to Post's version of the claim that it can't be that dependence requires things such as asymmetry and explanation, has, in the end, been unsuccessful. In order to hold onto the idea that psychophysical supervenience can entail psychophysical dependence in spite of what has been concluded in the previous section, one must try something else.

\subsection{Second attempt to rescue supervenience : dependence as supervenience}

A radical move to rescue the idea that psychophysical supervenience can entail psychophysical dependence, against the conclusions reached in $\S 4.3$, is that of

${ }^{27}$ The dependence of the argument to the effect that it can't be that dependence entails asymmetry on the claim that it can't be that dependence entails explanation emerges also in the argument that Post provides in defence of the holding of substitutivity in the context of his argument to the effect that it can't be that dependence requires asymmetry. In the argument in defence of substitutivity, in fact, Post makes use of the claim that there can be cases where determination holds 'by way of identity'. And - if what has been argued is right - this is the claim depending on rejecting the claim that dependence entails explanation. 
arguing that the notion of dependence is to be understood in terms of supervenience alone. ${ }^{28}$ The suggestion is that to say that the properties $A s$ depend on the properties $B s$ is to say that there can be no difference in the As without a difference in the $B s$; this, and nothing more, would be what dependence amounts to. ${ }^{29}$ If so, and if it is true that supervenience cannot entail asymmetry and explanation, it would follow that it can't be, after all, that dependence entails asymmetry and explanation. And even if supervenience cannot entail asymmetry and explanation, psychophysical supervenience would - quite trivially - entail psychophysical dependence.

The problem with this suggestion is that it is not clear how it could be grounded. Once again, it does not seem that we can appeal to clear-cut intuitions which go one way or the other. And it is possible to argue against the idea that the present suggestion can be supported on intuitive grounds, by appealing to the very fact that - as we have seen - there are people who do take dependence to be

${ }^{28}$ For this understanding of the notion of dependence, see, e.g., Kim (1978), and Kim (1979). There is, then, Hellman and Thompson's position, where the term 'determination' refers, in fact, to a supervenience thesis. (See Hellman \& Thompson (1975; 1977).)

${ }^{29}$ Note that, if determination is to be taken as the converse of dependence, the determination of the $A$ properties by the $B$ properties would - under the present conception of 'dependence' amount to the entailment of 'sameness in the $A$ properties' by 'sameness in the $B$ properties'. These analyses of the notions of dependence and determination can thus provide a way of satisfying the idea that determination involves a relation concerning something that is sufficient for something else, without rejecting the idea that determination can be regarded as the converse of dependence. (The idea that determination involves a relation concerning something which is sufficient for something else is suggested in Grimes (1991). Grimes, however, takes that as a reason for thinking that determination can not be taken as the converse of dependence - Cf. Grimes (1991), p. 83.) 
characterized by the Desiderata of $\S 4.3$. So, it is far from being intuitively obvious that dependence amounts to supervenience (alone) ${ }^{30}$

Moreover, in the psychophysical case, there are some further considerations which can be brought to bear against the idea that if $A$ supervenes on $B$, then $A$ depends on $B .{ }^{31}$ Under the assumption that determination is the converse of dependence, this idea is equivalent to the claim that if $A$ supervenes on $B$, then $B$ determines $A$. In the psychophysical case: if the mental supervenes on the physical, then the physical determines the mental. But by the determination of the mental by the physical what we mean - it seems - is that the physical facts are sufficient for the mental facts: given that the physical facts are thus and so, the mental facts will be thus and so. All we can say by appealing to supervenience, however, is, at best ${ }^{32}$, that given that the physical facts are thus and so, and given that the mental supervenes on the physical, then the mental facts will be thus and so. All we can get by appealing to supervenience - it seems - is thus, at best, the determination of the mental facts by the physical facts together with the fact that psychophysical supervenience holds. What we can't get, it can be argued, is the determination of the mental facts by the

\footnotetext{
${ }^{30}$ One might suggest that we might, here, in fact be dealing with two notions of dependence: on the one hand the one that amounts exclusively to supervenience; on the other, the one characterized by the Desiderata of $\S 4$.3. If so, the question to be asked (as it will be done in the next section) is then which of the two notions of dependence is the one that is in play, when considering the dependence requirement within the context of issues such as that of the relation between physicalism and supervenience. (Cf. what I said when discussing 'mutual dependence' in $\S 4.3$.)

${ }^{31}$ What follows is - I take it - a variant of an issue discussed in Crane (1991).

${ }^{32}$ I say 'at best' because we need (Th) to be true, here, given that what we want are conditionals of the same type of the Cs of $\S 3.4$.
} 
physical facts alone - i.e. the determination of the mental by the physical.

However, this is true only if the holding of the relation of psychophysical supervenience can't itself be taken as a physical fact. Psychophysical supervenience is, admittedly, a relation between the physical and the mental, and, hence, to this extent, it could be argued to fail to be a physical fact. But if the mental is reducible to the physical, then the featuring of the mental among the relata of psychophysical supervenience will no longer be a problem for taking the holding of psychophysical supervenience to be a physical fact.

So, as long as what we are working with is a reductive form of supervenience (cf. ch. 3), the kind of worry which has just been considered can be dissolved; the considerations above will not be effective against the idea that dependence can be analysed exclusively in terms of supervenience. What remains problematic, on the other hand, is the apparent lack of support for such an idea. Unless such a support can be found, the Desiderata of $\S 4.3$ remain problematic for the idea that psychophysical supervenience can entail psychophysical dependence.

\subsection{Problematic requirements and physicalism}

The idea that psychophysical supervenience can be used to express psychophysical dependence is, as I mentioned in the Introduction, very much related to the idea of using psychophysical supervenience to express physicalism. If there are problems with the idea that psychophysical supervenience can be used to express psychophysical dependence, there will be 
problems also with the idea of using psychophysical supervenience to express physicalism.

As I hinted at in the previous sections, however, it might well be that the notion of dependence relevant to physicalism is not the one characterized by the problematic requirements. I now want to address the issue directly by considering what the relationship between physicalism and the problematic requirements is.

Let me begin with the asymmetry requirement - i.e., in the psychophysical case, the idea that the mental must be asymmetrically related to the physical. The identity theorist - i.e. someone who maintains that every mental property is identical with a physical property - is a physicalist. So, at least when dealing with individual mental and physical properties, one can't associate physicalism in general with the idea that the mental stands to the physical in a relation which is asymmetric.

A close variant of the asymmetry requirement, however, is the antisymmetry requirement: Instead of requiring, for $A$ to be dependent on $B$, that $B$ be not dependent on $A$, what is required is that if $A$ is different from $B$ and $A$ depends on $B$, then $B$ is not dependent on $A ; A$ can be dependent on $B$ and $B$ dependent on $A$ as long as $A$ is identical to $B$. (Formally, a relation $R$ is antisymmetric iff $(\forall x)$ $(\forall y)(x \neq y \& R x y \rightarrow \sim R y x)$, or, put the other way round, iff $(\forall x)(\forall y)(R x y \&$ $R y x \rightarrow x=y)$.) The motivation behind taking dependence to be antisymmetric might be, once again, the idea that dependence entails explanation. This time, however, explanation would be assumed to be antisymmetric, as opposed to asymmetric.

If dependence requires antisymmetry, and if physicalism requires dependence, 
then physicalism requires antisymmetry. According to this picture, then, the physicalist is someone who maintains either that mental properties are identical to physical properties, and there is some mutual dependence between individual mental and physical properties, or that (i) mental properties are not identical to physical properties, (ii) every mental property is dependent on a physical property, (iii) no physical property is dependent on a mental property.

Supervenience is not only neither symmetric nor asymmetric, but it is also not antisymmetric. So, similarly to what happened with the asymmetry requirement, the satisfaction of the antisymmetry requirement cannot be ensured by supervenience in general.

However, if (Th) (see ch. 2) is true, it becomes possible to argue that the holding of an antisymmetric relation between the mental and the physical can follow from the holding of a relation of psychophysical supervenience. For if (Th) is true, it follows that any SSS form (i.e. any form of 'SupervenienceSatisfactorily-Supported') requires the holding of at least one of the Assumptions. And if any of the Assumptions holds, then either every mental property is identical to a physical property, or, every mental property is not identical to a physical property, but then mental properties stand to physical properties as $x$ stands to $y$ in $R x y$, and, given this equivalence, we can say $\sim R y x$. (Hence, as long as we are dealing with an SSS form, the holding of an antisymmetric relation between the mental and the physical does follow from the holding of a relation of psychophysical supervenience.)

That this is so can be seen by considering each of the Assumptions in turn. If $A 2$ holds, then every mental property is identical to a physical property. If, on the other hand, $A 3$ holds, then every mental property is not identical to a 
physical property, but then - given that if $A 3$ holds, mental properties will stand in a one-many, and, hence, asymmetric, relation with physical properties mental properties will stand to physical properties as $x$ stands to $y$ in $R x y$, and, given this equivalence, we can say $\sim R y x$. In the case of $A 1$, then, either every mental property is, in addition to being a property of a whole, also identical to a physical property, or mental properties are not identical to physical properties (i.e. there is no identity between properties of the wholes in question and the properties of the corresponding parts), but then the mental will stand to the physical as $x$ stands to $y$ in $R x y$, and, given this equivalence, it will be possible to say $\sim R y x$. (If mental properties are properties of certain wholes, and the properties of the parts of these wholes are physical properties, and there is no identity between mental and physical properties, then it will not be possible that mental properties are the properties of the parts of the wholes in question.)

We can thus conclude that if any of the Assumptions holds, the mental will stand to the physical in a relation which is antisymmetric. SSS forms entail antisymmetry. So, to sum up, we can say that, when considering individual mental and physical properties, the situation is as follows: Psychophysical supervenience cannot ensure that the mental stands to the physical in an asymmetric relation. But this is not a requirement which can be associated with physicalism in general. On the other hand, it might well be that physicalism is to be associated with a requirement of antisymmetry. But this is something whose satisfaction can be ensured by SSS forms.

Still, even if physicalism in general can't be taken to be associated with the asymmetry requirement when considering individual mental and physical properties, it might be that the situation is different when considering relata other 
than properties. Suppose, for example, that what we are considering are ways of picking out properties - i.e. we would be dealing with mental and physical predicates as opposed to mental and physical properties. Then, there would be room for an asymmetry claim even in the case of property identity. For property identity does not entail predicate identity. (Identity in the referents of predicates does not entail identity in the ways of picking out these referents, i.e. in the predicates themselves.) And, indeed, one might want to regard the physicalist as making an asymmetry claim. For a claim which is commonly regarded as the core of physicalism is that the physical is prior with respect to the mental. And such a 'priority' can be taken to be (also) an explanatory priority. ${ }^{33}$ Taking the relata of the relation of explanation to be predicates, that could then be couched as the idea that for all mental predications there are explanations in terms of physical predications, whereas the converse is not true. (That would amount to taking physicalism to be associated with the 'explanation requirement' under a conception of explanation as asymmetric.)

If such a claim ('the claim of explanatory priority', or 'CEP' for short) is to be regarded as characteristic of physicalism, then it is indeed true that - as the Sceptic would have it - there are problems with the idea of using supervenience to express physicalism. For CEP is an asymmetry claim. And supervenience is not asymmetric.

However, CEP is a claim involving epistemic and linguistic entities. So, there is room for arguing that the considered inadequacy of supervenience is due to our going beyond the merely metaphysical core of physicalist theses. As long as

${ }^{33}$ See, e.g., Post (1987; forthcoming); Melnyk (1991); Charles (1992); Papineau (1993). 
we remain within the non-epistemic/mind-independent sphere, there would be no problem with the idea of using supervenience to express physicalism.

Contrary to that, however, one can argue that even if we do remain within the non-epistemic/mind-independent sphere, there are, actually, problems with the idea of using supervenience to express physicalism. Consider, once again, the identity theorist - a prima facie counterexample to the idea that physicalism in general can be associated with an asymmetry claim. The identity theorist believes that every mental property is a physical property; and yet, for him, it is not equally true that every physical property is a mental property. In other words, identity is upheld between individual mental and physical properties, but not between the sets of the mental and physical properties; the set of the physical properties is meant to include, as a proper part, the set of the mental properties. This gives us asymmetry. And, indeed, an asymmetric relation which does not involve any epistemic entities.

As for properties, so for particulars. A physicalist who believes that every mental particular is a physical particular, will, at the same time, believe that it is not true that every physical particular is a mental particular. For him mental particulars cannot exist without physical particulars; but physical particulars can exist in the absence of mental particulars. (Note that the same kind of claim with 'properties' substituted for 'particulars' - is entailed by the claim of the identity theorist considered above.)

If mental properties supervene on physical properties in the way in which properties supervene in an NCMOF (if, for short, 'Smp'), then (i) it is not possible for mental particulars to exist without physical particulars; (ii) every mental particular is a physical particular; (iii) every mental property is physical 
in the weak sense of physicality considered in ch. $3 .{ }^{34}$ Now focus on (iii). (What I am going to say, however, is meant to apply, mutatis mutandis, also to (i) and (ii).) Given that supervenience is not asymmetric, from Smp it will not follow that physical properties do not supervene on mental properties in the way in which properties supervene in an NCMOF. $(\sim(\mathrm{Smp} \rightarrow \sim \mathrm{Spm})$.) From Smp, it will thus not follow either, that it is not the case that all physical properties are mental properties (in the sense that all the properties which are instantiated by physical particulars are properties which are instantiated by mental particulars). ${ }^{35}$

${ }^{34}$ To remind the reader, that was the sense of physicality according to which a property can count as physical as long as it is instantiated exclusively by particulars possessing properties which are used in the physical sciences.

${ }^{35}$ Proof:

key $S \mathrm{mp}=$ mental properties supervene on physical properties in the way in which properties supervene in an NCMOF.

$\mathrm{Spm}=$ physical properties supervene on mental properties in the way in which properties supervene in an NCMOF.

$\mathrm{Rmp}=$ all mental properties are physical properties.

$\mathrm{Rpm}=$ all physical properties are mental properties.

$\begin{array}{lll}\text { (1) } & \text { Smp } \rightarrow \text { Rmp } & \text { Premiss } \\ \text { (2) } & \text { Spm } \rightarrow \text { Rpm } & \text { Premiss } \\ \text { (3) } & \sim \text { Rpm } \rightarrow \sim \text { Spm } & 2 \text { Contraposition } \\ \text { (4) } & \sim(\text { Smp } \rightarrow \sim \text { Spm) } & \text { Premiss } \\ \text { (5) } & \text { Smp } \rightarrow \sim \text { Rpm } & \text { Ass } \\ \text { (6) } & \text { Smp } & \text { Ass } \\ \text { (7) } & \sim \text { Rpm } & 6,5 \rightarrow E \\ \text { (8) } & \sim \text { Spm } & 7,3 \rightarrow E \\ \text { (9) } \quad \text { Smp } \rightarrow \sim \text { Spm } & 6,8 \rightarrow \text { I } \\ \text { (10) } \quad \lambda & 4,9 \sim \text { E } \\ \text { (11) } & \sim(\text { Smp } \rightarrow \sim \text { Rpm }) & 5,10 \sim \text { I }\end{array}$


So, the conjunction of the claim that all mental properties are physical properties (Rmp) and that not all physical properties are mental properties $(\sim \mathrm{Rpm})$ does not follow from Smp.

If such a conjunction is, as many would agree ${ }^{36}$, required for physicalism, it follows that there are problems with the idea of using supervenience to express physicalism. Schematically: If $\square$ (Physicalism $\rightarrow$ Rmp \& $\sim$ Rpm), and if $\sim$ (Supervenience $\rightarrow$ Rmp \& $\sim$ Rpm), then $\sim$ (Supervenience $\rightarrow$ Physicalism).

However, suppose that it is assumed that $\sim$ Rpm holds as a matter of necessity. (In other words, what would be claimed is - under the present conception of 'being physical' and 'being mental' for properties - that panpsychism is necessarily false.) This would be the case if, for example, mental properties can be analysed in terms of, say, some causal roles which, necessarily, can't be instantiated by certain, very simple, physical properties. For, we may suppose, the impossibility of instantiating these causal roles is a necessary condition for being one of these physical properties; whatever can instantiate one of these causal roles can't count as one of these properties. If so, what would happen could be schematized as follows:

(i) $\square(\mathrm{Smp} \rightarrow \mathrm{Rmp}) \rightarrow \square(\mathrm{Smp} \rightarrow \mathrm{Rmp} \& \sim \mathrm{Rpm})$

(ii) $\square($ Smp $\rightarrow$ Rmp \& $\sim$ Rpm $) \rightarrow \square($ Smp $\rightarrow$ Physicalism $)$

(Assuming that (Rmp \& $\sim \mathrm{Rpm}$ ) is not only necessary but also sufficient for physicalism)

$$
\text { (iii) } \square(\mathrm{Smp} \rightarrow \mathrm{Rmp})
$$

$\therefore$ (iv) $\square($ Smp $\rightarrow$ Physicalism)

\footnotetext{
${ }^{36}$ See, e.g., Charles (1992); Jack (1994); Post (forthcoming).
} 
That shows that even if, given the identification of physicalism with an asymmetric claim, supervenience alone cannot entail physicalism, it is still true that this latter is entailed at least by a combination of a certain form of supervenience (i.e. an NCMOF) with some determinate assumptions (i.e. that $\sim \mathrm{Rpm}$, and that $\square(\mathrm{Rmp} \& \sim \mathrm{Rpm} \rightarrow$ Physicalism $))$. Given these assumptions, the form of supervenience in question does entail physicalism. By focusing on what supervenience does not entail, the Sceptics lead one to forget what supervenience does entail. This is, by contrast, what I want to draw people's attention to. In the present case, as in other cases considered in the previous chapters, it is something quite substantial. 


\section{Conclusion}

In the last two chapters, some of the implications of what has been argued in ch. 2 have cropped up: It has been shown that in virtue of (Th), the Cs of $\S 3.4$ can be derived from a supervenience claim, and an antisymmetric relation between individual properties can be ensured by supervenience. I now want to make some further comments on the bearing of the results of ch. 2 on the contents and expressive power of supervenience claims. In particular, I want to focus on two phenomena arising from (Th), i.e. from the fact that a supervenience claim cannot be satisfactorily supported unless it is grounded in one of the Assumptions.

First: This fact turns supervenience claims into intermediary stages in one's commitments, leading, in fact, to stronger commitments. If (Th) is true, and if one wants one's supervenience claims to be grounded, then one will, by endorsing a supervenience claim, commit oneself to the idea that one or another of the Assumptions holds. Indeed, in some cases, by using supervenience to express one's commitments, one will - as I am now going to illustrate - commit oneself to the idea that one or another of just some (as opposed to all) of the Assumptions holds. 
Consider, for example, the case of someone who wants to use supervenience to express the idea that the mental is 'nothing over and above' the physical. ${ }^{1}$ As we have seen in $\S 3.3$, the forms of supervenience suitable for this purpose is an NCMOF featuring two modal operators which ascribe absolute necessities. That means, then, that among the disjuncts of the disjunctive claim that one or another of the Assumptions holds, will not feature $A 3-D / D$, if this latter is understood, in the way mentioned in $\S 2.2$, as failing to entail a form of supervenience which features two necessity operators. Moreover, also any version of $A 3$ which is put, in the way considered in $\S 2.2$, in terms of $\mathrm{CP}$, is going to be ruled out. For - as we have seen, again, in $\S 2.2$ - the first modal operator of the form of supervenience entailed by any such version, ascribes only physical necessity. ${ }^{2}$

If, then, one wants the relata of the reduction entailed by one's supervenience claim to be intrinsic properties ${ }^{3}$, what's ruled out is going to be also any version of $A 3$ where the mental is analysed in terms of input/output dispositions which require extrinsic categorical bases. So, for example, suppose that one's desire for a glass of water is analysed as the disposition to reach for a

\footnotetext{
${ }^{1}$ See, e.g., Lewis (1983a); Chalmers (1996); Jackson (1998).

${ }^{2}$ I am assuming here that the set of the physically possible worlds is a subset of the set of all possible worlds.

${ }^{3}$ Although Kim now no longer believes that it is possible to get reduction by supervenience alone, I think that he can still be cited, in the present context, as an instance of someone who
} 
glass of $\mathrm{H}_{2} \mathrm{O}$ whenever one stands in a suitable perceptual relation with a glass of $\mathrm{H}_{2} \mathrm{O}$. If so, the categorical base of a desire for a glass of water is not going to be an intrinsic physical state. For all that the standing in an intrinsic physical state can entail is, at best, the disposition to reach for something which has the phenomenal character of water (but which is, maybe, a substance, $\mathrm{XYZ}$, that is not $\mathrm{H}_{2} \mathrm{O}$, and, hence, not water), whenever one stands in a suitable perceptual relation with something which has the phenomenal character of a glass of water. In order for one's state to entail the desire for a glass of water under the present analysis, one needs to fulfil further conditions such as that of being in an environment where what has the phenomenal character of water is $\mathrm{H}_{2} \mathrm{O}$. From that it follows that two individuals can differ in whether or not they have a desire for a glass of water even if they do not differ in their intrinsic physical states. In other words, and generalizing, analyses of mental states in terms of input/output dispositions which require extrinsic categorical bases do not yield forms of supervenience where the subvenient properties are intrinsic. Given that, on the contrary, this is precisely what is needed for the relata of a reduction entailed by one's supervenience claim to be intrinsic, it follows that, as I have claimed, any version of $A 3$ where the mental is analysed in terms of input/output dispositions requiring extrinsic categorical bases will not feature 
among the possible grounds of a supervenience claim which is meant to entail the kind of reduction under discussion.

Moreover, suppose that by making a supervenience claim one wants to commit oneself to something more than there being a reductive relation between the whole set of the mental properties of an individual and the whole set of the physical properties of this individual. ${ }^{4}$ One might believe, for instance, in the existence of some kind of reductive relation between individual mental and physical properties. If so, it won't be possible to ground the relevant kind of supervenience in the kind of derivation considered in $\S 2.2$ when discussing $A 3$ unless individual mental states are analysed exclusively in terms of physical inputs and outputs. (As it will be remembered, this type of analysis of the mental was necessary for concluding, via $\mathrm{CP}$, that the mental supervenes on the physical.) A functionalist analysis of the mental, for instance, will thus have to be excluded as something suitable to ground the kind of reduction wanted. For a functionalist analysis of individual mental states typically includes mental states among the inputs and outputs. All that, according to the functionalist, can be analysed exclusively in terms of physical inputs and outputs is the whole set of the mental states of an individual..$^{5}$ Once more, then, we are cutting down in the range of Assumptions which can ground a

\footnotetext{
${ }^{4}$ Again, I think that the later Kim can be cited as an example. (See, again, Kim (1998), Ch. 1 \& Ch. 4.)

${ }^{5}$ See, e.g., Lewis (1994a), p. 416.
} 
supervenience claim suitable to give one what one wants. And what has been argued in ch. 2 can then be put to work to bring out some otherwise more or less hidden commitments. (E.g. The claim of a reductive relation between individual mental and physical properties can, if expressible only in terms of supervenience, be grounded only if one is willing to go for something at least as strong as behaviourism; anything weaker, such as functionalism, won't do.)

All of that speaks, of course, against some of the Enthusiasts' hopes. For it is doubtful that at least the early Enthusiasts would have welcome the idea that a supervenience claim commits one to one or another of the Assumptions (or, even worse, to one or another of just some of the Assumptions). For them, the hope was precisely that to get what they wanted, supervenience was all that they needed to commit themselves to. ${ }^{6}$

But the fate of supervenience as an interesting concept need not be bound to what the early Enthusiasts had in mind. And if I am right about (Th), any use that could be made of supervenience is in fact going to be available rather to those who are not unwilling to commit themselves to one or another of the Assumptions. I now want to consider what can be said in favour of supervenience within this context, and in connection with the second phenomenon arising from (Th) that I said I would have discussed.

\footnotetext{
${ }^{6}$ See, e.g., Haugeland $(1982 ; 1984)$.
} 
Even in the cases considered above, where by expressing certain (reductive) claims in terms of supervenience one ends up committing oneself to one or another of just some (as opposed to all) of the Assumptions, the commitment implied by the relevant kind of supervenience remains of a disjunctive form: From the fact that the relevant kind of supervenience cannot be grounded in some of the Assumptions, it does not follow that what one ends up with is a single Assumption. This committing oneself to a claim of a disjunctive form is the second phenomenon arising from $(\mathrm{Th})$ that I want to consider. I now want to argue that it provides room for using supervenience in an interesting way.

Suppose that one wants to be a physicalist. ${ }^{7}$ The motivations for that might be the mixed bag discussed in Haugeland (1984). ${ }^{8}$ Or they might be more focused ones having to do with one's beliefs about the causal structure of the world. ' Whatever the motivations, anyway, this person (call it ' $\mathrm{X}$ ') wants to subscribe to some general claims, such as that the mental depends on the physical. Some would then argue that, in order to be a physicalist, one needs to subscribe also to some claims such as that the mental is nothing over and above

\footnotetext{
${ }^{7}$ The position I am about to sketch corresponds, to a certain extent, to what Lewis calls 'minimal materialism'. (See Lewis (1983a), p. 361.)

${ }^{8}$ Haugeland considers ideas such as the appeal to simplicity and unity, together with what he calls 'fear of darkness', and 'the way the wind blows'. (See Haugeland (1984), pp. $3 \mathrm{ff}$.)

${ }^{9}$ See, e.g., Lewis (1966); Armstrong (1968); Papineau (1993).
} 
the physical. ${ }^{10}$ What is important, in any event, is that there is a determinate set of claims, of the kind mentioned, that $\mathrm{X}$ wants to subscribe to. On the other hand, though, $\mathrm{X}$ does not want to commit itself to any detailed account of the relation between the mental and the physical. Is the relation between mental and physical properties of the same kind as the one that holds between the properties of a whole and the properties of its parts? Are mental properties higher-order properties with respect to physical properties? And, if so, what kind of higher-order characterization should be given to mental properties? X does not know. It wants to withhold judgment on that (maybe because it thinks that what has been said so far is not conclusive one way as opposed to the other). All that it wants to rule out are some accounts of the relation between the mental and the physical which it believes to be inadequate. But, for $\mathrm{X}$, the issue of which of the alternatives left is the correct one is to be left open.

Supervenience claims provide the means for expressing this type of position - i.e. the position of someone who wants to subscribe to a determinate set of claims (which are then going to be the ones entailed by the relevant form of supervenience), and, at the same time, withhold judgment on some others. By making one's supervenience claims more or less specific (i.e. by being more or less specific about which form of supervenience one has in mind), one can then, of course, make more or less specific also the claims which are entailed by

\footnotetext{
${ }^{10}$ See, e.g., Smart (1976); Armstrong (1980); Lewis (1983a; 1994a); Chalmers (1996); Jackson
} 
supervenience and those which are required by it in order for it to be grounded. (As we have seen, for example, only some forms of supervenience can give us certain types of reductive relations, and to ground these forms of supervenience only some of the Assumptions will do.) To this extent, one can tailor one's claim of supervenience to suit the level of one's commitments. And this flexibility of the concept can be regarded as one of its virtues.

The Sceptics frequently complain about the weakness of supervenience. Consider, for instance, what it is argued in Kim (1998). Kim blames the consistency of supervenience with 'a host of classic positions on the mind-body problem $^{\prime 11}$ for the (alleged) failure of supervenience to provide a mind-body theory.

But, firstly, if what one wants is a strong claim, then it is not clear that supervenience can't help. In making the claim mentioned, what Kim has in mind must clearly be a very general claim of supervenience, something like 'the mental supervenes on the physical'. Only in this way, in fact, what Kim says can be true of all the 'classic positions' he is considering. For among these positions features, for example, even substance dualism. ${ }^{12}$ And, as we have seen, there are forms of supervenience (i.e. NCMOFs) which are inconsistent with substance dualism. As long as one makes one's claim of supervenience

(1998).

\footnotetext{
${ }^{11} \mathrm{Kim}$ (1998), p. 12.

${ }^{12}$ See Kim (1998), note 20, p. 123.
} 
determinate (by specifying what particular form of supervenience one has in mind), one can rule out much more than what is conceded by Kim.

On the other hand, a certain amount of 'weakness' is precisely what is needed to discharge the job for which Kim concedes that supervenience is suitable. The idea is that of viewing supervenience as capturing the core commitment of 'all positions on the nature of mentality that are basically physicalistic' ${ }^{13}$ As such, supervenience will have to be compatible with all these positions without, at the same time, being identifiable with any of them to the exclusion of the others. ${ }^{14}$

As long as one's form of supervenience is weak/general enough, it can discharge this type of job (which is in fact very close to the idea, described above, of capturing the position of someone who wants to commit oneself to a determinate set of claims without, however, taking any stand on some more specific ones). On the other hand, by making one's claim of supervenience more specific, one can - as we have seen in the case of certain reductive claims - use the notion of supervenience also to express stronger claims. I thus agree with the Enthusiast that there are interesting things supervenience can do. Given what I argued in the previous chapters, however, the notion of

\footnotetext{
${ }^{13}$ Kim (1998), p. 14. This claim of Kim strikes one as being at odds with his previous claim that supervenience is compatible with substance dualism. However, what that can be taken to suggest is that Kim has, in fact, different concepts of supervenience in mind when he makes his claims.

${ }^{14}$ Cf. Lewis (1983a), p. 361.
} 
supervenience which is suitable to do what has been mentioned is going to be quite different from the one that at least some of the Enthusiasts have in mind. For, in order to be grounded, it will have to entail one or another of the Assumptions; and in order to express certain (e.g. reductive) claims, it will have to be characterized by features (e.g. strong necessity), and/or accompanied by a package of metaphysical assumptions, which are not congenial to all. Supervenience, thus, can be useful, but maybe only to a happy few. 


\section{References}

Alexander, S. (1920). Space, Time, and Deity. London: Macmillan.

Armstrong, D.M. (1968). A Materialist Theory of Mind. London: Routledge and Kegan Paul.

(1978). Universals and Scientific Realism. Cambridge: Cambridge University Press.

(1980). 'Naturalism, Materialism, and First Philosophy', in D.M. Armstrong, The Nature of Mind. St. Lucia, Queensland: University of Queensland Press, pp. 149-65.

Audi, R. (1993). 'Mental Causation: Sustaining and Dynamic', in J. Heil (ed.), Mental Causation, New York: Clarendon Press, pp. 53-74.

Bacon, J. (1986). 'Supervenience, Necessary Coextension, and Reducibility'. Philosophical Studies 49: 163-76.

(1995). 'Weak Supervenience Supervenes', in E.E. Savellos \& U.D. Yalçin (eds.), Supervenience: New Essays, Cambridge: Cambridge University Press, pp. 101-9.

Beckermann, A. (1992). 'Introduction - Reductive and Nonreductive Physicalism', in A. Beckermann, H. Flohr, J. Kim (eds.), Emergence or Reduction?, Berlin/New York: de Gruyter, pp. 1-21.

Blackburn, S. (1984). Spreading the Word. Oxford: Clarendon Press.

Bonevac, D. (1995). 'Reduction in the Mind of God', in E.E. Savellos \& U.D. Yalçin (eds.), Supervenience: New Essays, Cambridge: Cambridge University Press, pp. 124-39.

Burks, A.W. (1951). 'The Logic of Causal Propositions'. Mind 60: 363-82. 
Chalmers, D. (1996). The Conscious Mind. New York: Oxford University Press.

Charles, D. (1992). 'Supervenience, Composition, and Physicalism', in D. Charles \& K. Lennon (eds.), Reduction, Explanation, and Realism, Oxford: Oxford University Press, pp. 265-96.

Churchland, Patricia (1986). Neurophilosophy. Cambridge, Massachusetts: MIT Press.

Conee, E. (1995). 'Supervenience and Intentionality', in E.E. Savellos \& U.D. Yalçin (eds.), Supervenience: New Essays, Cambridge: Cambridge University Press, pp. 273-92.

Crane, T. (1991). 'All God Has to Do'. Analysis 51: 235-44.

(1995). 'The Mental Causation Debate'. Proceedings of the Aristotelian Society Supplementary Volumes 69: 211-36.

(forthcoming). 'The Significance of Emergence'.

Davidson, D. (1967). 'Causal Relations'. Journal of Philosophy 64: 691-703. Reprinted in D. Davidson, Essays on Actions and Events, Oxford: Clarendon Press, 1980, pp. 149-62. (Page references are to this latter edition.)

(1969). 'The Individuation of Events', in N. Rescher \& D. Reidel (eds.), Essays in Honor of Carl G. Hempel, Dordrecht: D. Reidel Publishing Company, pp. 216-34. Reprinted in D. Davidson, Esssays on Actions and Events, Oxford: Clarendon Press, 1980, pp. 163-80. (Page references are to this latter edition.)

(1970a). 'Mental Events', in L. Foster and J.W. Swanson (eds.), Experience and Theory, Amherst: The University of Massachusetts Press and 
Duckworth, pp. 79-101. Reprinted in D. Davidson, Essays on Actions and

Events, Oxford: Clarendon Press, 1980, pp. 207-25. (Page references are to this latter edition.)

(1970b). 'Events as Particulars'. Nô̂s 4: 25-32. Reprinted in D. Davidson,

Essays on Actions and Events; Oxford: Clarendon Press, 1980, pp. 181-87.

(Page references are to this latter edition.)

(1971). 'Eternal vs. Ephemeral Events'. Noûs 5: 335-49. Reprinted in D.

Davidson, Essays on Actions and Events, Oxford: Clarendon Press, 1980, pp. 189-203. (Page references are to this latter edition.)

(1973). 'The Material Mind', in P. Suppes, L. Henkin, G.C. Moisil, and A. Joia (eds.), Proceedings of the Fourth International Congress for Logic, Methodology, and Philosophy of Science, North Holland Publishing Company, pp. 709-22. Reprinted in D. Davidson, Essays on Actions and Events, Oxford: Clarendon Press, 1980, pp. 245-59. (Page references are to this latter edition.)

(1993). 'Thinking Causes', in A. Mele and J. Heil (eds.), Mental Causation, New York: Oxford University Press, pp.1-10.

DePaul, M. (1987). 'Supervenience and Moral Dependence'. Philosophical Studies 51: 425-39.

Dretske, F. (1998). 'Minds, Machines, and Money: What Really Explains Behavior', in J. Bransen and S. E. Cuypers (eds.), Human Action, Deliberation, and Causation, Dordrecht: Kluwer Academic Publishers.

Enç, B. (1986). 'Essentialism without Individual Essences: Causation, Kinds, Supervenience, and Restricted Identities.' Midwest Studies in Philosophy 11: 403-26. 
Field, H. (1989). Realism, Mathematics and Modality. Oxford: Basil Blackwell. Fodor, J. (1974). 'Special Sciences or: the Disunity of Science as a Working Hypothesis'. Synthese 28: 97-115.

Goodman, N. (1955). Fact, Fiction, and Forecast. Cambridge, Massachusetts: Harvard University Press.

Grimes, T.R. (1988). 'The Myth of Supervenience'. Pacific Philosophical Quarterly 69: 152-60.

(1991). 'Supervenience, Determination, and Dependency'. Philosophical Studies 62: 81-92.

Hare, R.M. (1952). The Language of Morals. London: Oxford University Press. Haugeland, J. (1982). 'Weak Supervenience'. American Philosophical Quarterly 19: 93-104.

(1984). 'Ontological Supervenience'. Southern Journal of Philosophy Supplementary Volumes 22: 1-12.

Heil, J. (1998). 'Supervenience Deconstructed'. European Journal of Philosophy 6: 146-55.

Hellman, G.P. (1985). 'Determination and Logical Truth'. Journal of Philosophy 82: 607-16.

Hellman, G.P., \& Thompson, F.W. (1975). 'Physicalism: Ontology, Determination, and Reduction'. Journal of Philosophy 72: 551-64. (1977). 'Physicalist Materialism'. Noûs11: 309-45.

Hempel, C. (1965). Aspects of Scientific Explanation and Other Essays in the Philosophy of Science. New York: Free Press.

(1969). 'Reduction: Ontological and Linguistic Facets', in S. Morgenbesser, P. Suppes, M. White (eds.), Philosophy, Science, and Method, New York: 
St. Martin's Press, pp. 179-99.

Hempel, C. \& Oppenheim, P. (1948). 'The Logic of Explanation'. Philosophy of Science 15: 135-75.

Hooker, C. (1981). 'Towards a General Theory of Reduction'. Dialogue 20: 38$59 ; 201-36 ; 496-529$.

Horgan, T. (1982). 'Supervenience and Microphysics'. Pacific Philosophical Quarterly 63: 29-43.

(1984). 'Supervenience and Cosmic Hermeneutics'. Southern Journal of Philosophy Supplementary Volumes 22: 19-38.

(1987). 'Supervenient Qualia'. Philosophical Review 96: 491-520.

(1989). 'Mental Quausation'. Philosophical Perspectives 3: 47-76.

(1993). 'From Supervenience to Superdupervenience: Meeting the Demands of a Material World'. Mind 102: 555-86.

Jack, A. (1994). 'Materialism and Supervenience'. Australasian Journal of Philosophy 72: 426-44.

Jackson, F. (1998). From Metaphysics to Ethics. Oxford: Clarendon Press.

Johnson, W. E. (1921). Logic. London: Cambridge University Press.

Kemeny, J.G. \& Oppenheim, P. (1956). 'On Reduction', Philosophical Studies 7: 6-19.

Kim, J. (1973). 'Causation, Nomic Subsumption, and the Concept of Event'. Journal of Philosophy 70: 217-36. Reprinted in J. Kim, Supervenience and Mind, New York: Cambridge University Press, 1993, pp. 3-21. (Page references are to this latter edition.)

(1976). 'Events as Property Exemplifications', in M. Brand \& D. Walton (eds.), Action Theory, Dordrecht: D. Reidel Publishing Co., pp. 159-77. 
Reprinted in J. Kim, Supervenience and Mind, New York: Cambridge University Press, 1993, pp. 33-52. (Page references are to this latter edition.)

(1978). 'Supervenience and Nomological Incommensurables', American Philosophical Quarterly 15: 149-56.

(1979). 'Causality, Identity, and Supervenience in the Mind-Body Problem'. Midwest Studies in Philosophy 4: 31-49.

(1982). 'Psychophysical Supervenience'. Philosophical Studies 41: 51-70. Reprinted in J. Kim, Supervenience and Mind, New York: Cambridge University Press, 1993, pp. 175-93. (Page references are to this latter edition.)

(1984a). 'Concepts of Supervenience'. Philosophy and Phenomenological Research 45: 153-76. Reprinted in J. Kim, Supervenience and Mind, New York: Cambridge University Press, 1993, pp. 53-78. (Page references are to this latter edition.)

(1984b). 'Epiphenomenal and Supervenient Causation', Midwest Studies in Philosophy 9: 257-70. Reprinted in J. Kim, Supervenience and Mind, New York: Cambridge University Press, 1993, pp. 92-108. (Page references are to this latter edition.)

(1987). " "Strong" and "Global" Supervenience Revisited'. Philosophy and Phenomenological Research 48: 315-26. Reprinted in J. Kim, Supervenience and Mind, New York: Cambridge University Press, 1993, pp. 79-91. (Page references are to this latter edition.)

(1988). 'Supervenience for Multiple Domains'. Philosophical Topics 16: 129-

50. Reprinted in J. Kim, Supervenience and Mind, New York: Cambridge 
University Press, 1993, pp. 109-30. (Page references are to this latter edition.)

(1989a). 'The Myth of Nonreductive Materialism'. Proceedings and Addresses of the American Philosophical Association 63: 31-47. Reprinted in J. Kim, Supervenience and Mind, New York: Cambridge University Press, 1993, pp. 265-84. (Page references are to this latter edition.)

(1989b). 'Mechanism, Purpose, and Explanatory Exclusion'. Philosophical Perspectives 3: 77-108. Reprinted in J. Kim, Supervenience and Mind, New York: Cambridge University Press, 1993, pp. 237-64. (Page references are to this latter edition.)

(1990a). 'Supervenience as a Philosophical Concept'. Metaphilosophy 21: 127. Reprinted in J. Kim, Supervenience and Mind, New York: Cambridge University Press, 1993, pp. 131-60. (Page references are to this latter edition.)

(1990b). 'Explanatory Exclusion and the Problem of Mental Causation', in E. Villanueva (ed.), Information, Semantics, and Epistemology, Cambridge: Blackwell, pp. 36-56.

(1992). " "Downward Causation" in Emergentism and Non-reductive Physicalism', in A. Beckermann, H. Flohr, J. Kim (eds.), Emergence or Reduction?, Berlin/New York: de Gruyter, pp. 119-38.

(1993). Supervenience and Mind. New York: Cambridge University Press. (1997a). 'The Mind-Body Problem: Taking Stock After Forty Years'. Philosophical Perspectives 11: 185-207.

(1997b). 'Does the Problem of Mental Causation Generalize?'. Proceedings of the Aristotelian Society 97: 281-99. 
(1998). Mind in a Physical World. Cambridge, Massachusetts: The MIT Press.

LePore, E. \& Loewer, B. (1987). 'Mind Matters'. Journal of Philosophy 84: $630-42$.

Lewis, D. (1966). 'An Argument for the Identity Theory'. Journal of Philosophy 63: 17-25. Reprinted in D. Lewis, Philosophical Papers Vol. I, New York: Oxford University Press, 1983, pp. 99-107. (Page references are to this latter edition.)

(1983a). 'New Work for a Theory of Universals'. Australasian Journal of Philosophy, 61: 343-77.

(1983b). Philosophical Papers Vol. I. New York: Oxford University Press.

(1986a). Philosophical Papers Vol. II. New York: Oxford University Press.

(1986b). On the Plurality of Worlds. Oxford: Blackwell.

(1994a). 'Lewis, David: Reduction of Mind', in S. Guttenplan (ed.), A Companion to the Philosophy of Mind. Oxford: Blackwell, pp. 412-31.

(1994b). 'Chance and Credence: Humean Supervenience Debugged'. Mind 103: 473-90.

Loewer, B. (1995). 'An Argument for Strong Supervenience', in E.E. Savellos and U.D. Yalçin (eds.), Supervenience: New Essays, Cambridge: Cambridge University Press, pp. 218-25.

Macdonald, C. (1995). 'Psychophysical Supervenience, Dependency, and Reduction', in E.E. Savellos \& U.D. Yalçin (eds.) Supervenience : New Essays. Cambridge: Cambridge University Press, pp. 140-57.

Macdonald, C. \& Macdonald G. (1986). 'Mental Causes and Explanation of Action', in L. Stevenson, R. Squires, J. Haldane (eds.), Mind, Causation, and Action, Oxford: Blackwell, pp. 35-48. 
Marras, A. (1994). 'Nonreductive Materialism and Mental Causation'. Canadian Journal of Philosophy 24: 465-93.

Martin, C.B. (1996). 'Properties and Dispositions', in D.M. Armstrong, C.B. Martin, and U.T. Place, Dispositions, London: Routledge, pp. 71-87.

McLaughlin, B. (1984). 'Event Supervenience and Supervenient Causation'. Southern Journal of Philosophy Supplementary Volumes 22: 71-91.

(1995). 'Varieties of Supervenience', in E.E. Savellos and U.D. Yalçin (eds.), Supervenience: New Essays, Cambridge: Cambridge University Press, pp. 16-59.

Mellor, D.H. (1991). Matters of Metaphysics. New York: Cambridge University Press.

(1993). 'Supervenience? No Chance! Reply to Menuge'. Analysis 53: 236-9.

Melnyk, A. (1991). 'Physicalism: from Supervenience to Elimination'. Philosophy and Phenomenological Research 51: 573-87.

(1995). 'Physicalism, Ordinary Objects, and Identity'. Journal of Philosophical Research 20: 221-35.

(1997). 'On the Metaphysical Utility of Claims of Global Supervenience'. Philosophical Studies 87: 277-308.

Menuge, A. (1993). 'Supervenience, By Chance? Reply to Crane and Mellor'. Analysis 53: 228-35.

Menzies, P. (1988). ‘Against Causal Reductionism'. Mind 97: 551-74.

Moore, G.E. (1922). Philosophical Studies. London: Routledge \& Kegan Paul.

Moser, K. \& Trout, J.D. (1995). 'Physicalism, Supervenience, and Dependence', in E.E. Savellos \& U.D. Yalçin (eds.) Supervenience: New Essays, Cambridge: Cambridge University Press, pp. 187-217. 
Nagel, E. (1951). 'Mechanistic Explanation and Organismic Biology', Philosophy and Phenomenological Research11: 327-38.

(1961). The Structure of Science. London: Routledge \& Kegan Paul.

Nozick, R. (1981). Philosophical Explanations. Cambridge, Massachusetts: Harvard University Press.

Oddie, G., and Tichy, P. (1990). 'Resplicing Properties in the Supervenience Base'. Philosophical Studies 58: 259-69.

Oliver, A. (1996). 'The Metaphysics of Properties'. Mind 105: 1-80.

Owens, D. (1989). 'Disjunctive Laws'. Analysis 49: 197-202.

Papineau, D. (1993). Philosophical Naturalism. Oxford: Blackwell. (1995). 'Arguments for Supervenience and Physical Realization', in E.E. Savellos \& U.D. Yalçin (eds.), Supervenience: New Essays, Cambridge: Cambridge University Press, pp. 226-43.

Paull, C.P., \& Sider, T.R. (1992). 'In Defense of Global Supervenience'. Philosophy and Phenomenological Research, 32: 330-45.

Peacocke, C. (1993). 'Externalist Explanation'. Proceedings of the Aristotelian Society 93: 203-30.

Petrie, B. (1987). 'Global Supervenience and Reduction'. Philosophy and Phenomenological Research 48: 119-30.

Post, J.F. (1987). The Faces of Existence. Ithaca, N.Y.: Cornell University Press.

(1995). ' "Global” Supervenient Determination: Too Permissive?', in E.E. Savellos \& U.D. Yalçin (eds.) Supervenience: New Essays, Cambridge: Cambridge University Press, pp. 73-99. (forthcoming). 'Versus Asymmetric Supervenient Determination'. 
Prior, A.N. (1949). 'Determinables, Determinates and Determinants', Mind 58: $1-20 ; 178-94$.

Quine, W.V.O. (1953). 'Two Dogmas of Empiricism', in W.V.O. Quine, From a Logical Point of View. Cambridge, Massachusetts: Harvard University Press, pp. 20-46.

(1981). 'Things and their Place in Theories', in W.V.O. Quine, Theories and Things, Cambridge, Massachusetts: Harvard University Press, pp. 1-23.

(1985). 'Events and Reification', in E. LePore \& B. McLaughlin (eds.), Actions and Events: Perspectives on the Philosophy of Donald Davidson, Oxford: Blackwell, pp. 162-71.

Salmon, W.C. (1970). 'Statistical Explanation', in W.C. Salmon, R.C. Jeffrey, and J.G. Greeno, Statistical Explanation and Statistical Relevance, University of Pittsburgh Press, pp. 29-87.

(1998). Causality and Explanation. New York: Oxford University Press.

Savellos, E.E. \& Yalçin, U.D. (1995). 'Introduction', in E.E. Savellos \& U.D. Yalçin (eds.), Supervenience: New Essays, Cambridge: Cambridge University Press, pp. 1-15.

Seager, W. (1988). 'Weak Supervenience and Materialism'. Philosophy and Phenomenological Research 48: 697-709.

Searle, J. (1959). 'Determinables and the Notion of Resemblance'. Proceedings of the Aristotelian Society Supplementary Volumes 33: 141-58.

Segal, G. \& Sober, E. (1991). 'The Causal Efficacy of Content'. Philosophical Studies 63: 1-30.

Smart, J.J.C. (1976). 'The Revival of Materialism'. The Listener 95: 535-9. Reprinted in J.J.C. Smart, Essays Metaphysical and Moral, Oxford: 
Blackwell, 1987, pp. 240-5. (Page references are to this latter edition.)

Stalnaker, R. (1996). 'Varieties of Supervenience', Philosophical Perspectives 10: $221-41$.

Stephan, A. (1992). 'Emergence - A Systematic View on its Historical Facets', in A. Beckermann, H. Flohr, J. Kim (eds.), Emergence or Reduction?, Berlin/New York: de Gruyter, pp. 25-48.

Teller, P. (1984a). 'A Poor Man's Guide to Supervenience and Determination', Southern Journal of Philosophy 22: 137-67.

(1984b). 'Comments on Kim's Paper'. Southern Journal of Philosophy 22: $57-61$.

Van Cleve, J. (1990). 'Mind - Dust or Magic? Panpsychism versus Emergence.' Philosophical Perspectives 4: 215-26.

Von Kutschera, F. (1992). 'Supervenience and Reduction'. Erkenntnis 36: 33343.

Yablo, S. (1992). 'Mental Causation'. The Philosophical Review 101: 245-80.

Zangwill, N. (1996). 'Good Old Supervenience: Mental Causation on the Cheap'. Synthese 106: 67-101. 


\section{Abbreviations and Definitions}

A1: Mental properties can be taken to be properties of (some) wholes, and the properties of the parts of these wholes are physical properties

A2: Mental properties are such that they can be identified with physical properties

A3: Mental properties and physical properties are such as to stand in the kind of relation which holds between, respectively, a property which is picked out by a higher-order characterization (i.e. by a predication which is entailed by a number

of, 'lower-order', predications) and the properties which are picked out by the lower-order predications entailing the higher-order predication in question

ACEM = The Argument from the Causal Efficacy of the Mental

APWS $=$ The Analytically Possible Worlds

$\mathbf{B E}=$ The relation which holds between two sets of properties when the instantiation of properties from one set is the Best Explanation for the instantiation of properties from the other

BDs = Behavioural Differences

$\mathbf{B} / \mathbf{N}=$ The distinction between Broad and Narrow dependence 
CCP = The Causal Closure of the Physical: Any physical effect can be completely accounted for in terms of the causal efficacy, with regard to it, of certain physical properties

CCS = Causal Conditionals / Supervenience combination

CEE = Causal Explanatory Exclusion: For any effect there is no more than a single complete and independent causal explanation

CEM = The Causal Efficacy of the Mental: There are causal transactions with the physical which take place in virtue of the mental properties of the relata CEP = The Claim of Explanatory Priority: The physical is explanatorily prior with respect to the mental

CMOFs = Modal-Operator Forms of supervenience where the subvenient sets are Closed under conjunction and complementation

$\mathbf{C} / \mathbf{N}=$ The 'Contingent/Necessary distinction', i.e. the distinction between relations of covariance which hold as a matter of contingency, and relations of covariance which hold as a matter of necessity

$\mathbf{C P}=$ The Completeness of Physics: Physics is complete; for any physical phenomenon there exists a complete physical explanation

$\mathbf{C R}=$ Closure under Resplicing: Where $\varphi_{\mathrm{w}}$ is the extension of [a property $\varphi$ at world $w$, and $B_{w}=\left\{\varphi_{\mathrm{w}}: \varphi \in B\right\}, B$ is also to contain any property $\psi$ such that $\psi_{\mathrm{w}}$ $\in B_{w}$ for each world $w$ 
Cs $=$ Conditionals of form $(\forall x)(G x \rightarrow F x)$ which do not rest on the prior assumption that some individual is $F$ and, for this reason, also $G$ D = Detectability principle: Things (by which it is meant objects, phenomena, etc.) are detectable only in virtue of physical manifestations (by which it is meant manifestations which are describable by appealing to predicates which are used in the physical sciences)

DI $=$ One's Degree of Intelligence

DMD = One's Degree of Manual Dexterity

$\mathbf{E}=$ The relation of Explanation which is implied by dependence

ES = Event-Supervenience

GDF $=$ Some Genetic and Developmental Factors

$\mathbf{G} / \mathbf{L}=$ The distinction between Global and Local forms of supervenience

GS = Global Supervenience: A set of properties $A$ globally supervenes on a set of properties $B$ iff worlds which are $B$-indiscernible are also $A$-indiscernible $\mathbf{H}=$ Homogeneity: 'causal efficacy' means the same when the concept is applied to the mental as when the concept is applied to the physical

ILTE $=$ Inter-Level-Theory-Explanation

Local Supervenience: A set of properties A locally supervenes on a set of properties $B$ iff individuals who are $B$-indiscernible are also $A$-indiscernible LPWS $=$ The Logically Possible Worlds 
MDS = Forms of Supervenience for Multiple Domains: Forms of supervenience where it is left open whether we have the same domain for both $A$-distributions and $B$-distributions

MOFs = Modal-Operator Forms of supervenience MOT $=$ Modal-Operator Talk

MPS $=$ The claim that Mental properties Supervene on Physical properties MS = Mereological Supervenience: [A macoproperty] $F$ mereologically supervenes on [a micro(based)-property] $m(F)$ iff it is nomologically necessary that if any object, $x$, has $m(F)$ at any time, $t$, then $x$ has $F$ at $t$.

NCMOFs = Modal-Operator Forms of supervenience where the subvenient sets are Not Closed under conjunction and complementation NCSS $=$ Forms of Strong Supervenience whose sets are Neither Closed nor semi-closed under identity and quantification

NHA $=$ No-Homogeneity Account, i.e. an account of the causal efficacy of the mental which (a) is satisfactory, and (b) is compatible with a rejection of $(\mathrm{H})$ NRSS $=$ Forms of Strong Supervenience whose sets are Not closed under Resplicing $\mathbf{P C}=$ The Petrie Case PD = Property Dualism: Mental properties cannot be identified with physical properties 
$\mathbf{P E}=$ Probabilifying Explanation: A kind of explanation characterized by the explanans's making the explanandum sufficiently probable - i.e. reaching the degree of probability (generally greater than 0.5 ) which is judged sufficient for having an explanation

PRS $=$ Predicate-Supervenience

PS = Property-Supervenience

PWFs $=$ Possible-World Forms of supervenience

PWT $=$ Possible-World Talk

Regional Supervenience: A set of properties $A$ regionally supervenes on a set of properties $B$ iff world-regions (i.e. portions of worlds corresponding to subsets of the sets of the inhabitants of the various possible worlds) which are $B$ indiscernible are also $A$-indiscernible RSS $=$ Forms of Strong Supervenience where the subvenient sets are closed under Resplicing

SCSS $=$ Forms of Strong Supervenience where the subvenient sets are Semiclosed under identity and quantification SDS $=$ Single Domain Supervenience: Forms of supervenience where we have the same domain for both $A$-distributions and $B$-distributions

SBGS = Similarity-Based Global Supervenience: A set of properties $A$ is related by SBGS to a set of properties $B$ iff any two worlds that are pretty much similar in respect of $B$ are pretty much similar in respect of $A$ 
SGS = Strong forms of Global Supervenience, i.e. forms of global supervenience which require the yielding of $A$-indiscernibility by $B$ indiscernibility under any mapping

SS1: A set of properties $A$ strongly supervenes on a set of properties $B$ iff for any worlds $w_{j}$ and $w_{k}$, and for any objects $x$ and $y$, if $x$ in $w_{j}$ is $B$-indiscernible from $y$ in $w_{k}$, then $x$ in $w_{j}$ is $A$-indiscernible from $y$ in $w_{k}$

SS2: A set of properties $A$ strongly supervenes on a set of properties $B$ iff, necessarily, for each $x$ and each property $F$ in $A$, if $x$ has $F$, then there exists a property $G$ in $B$ such that $x$ has $G$, and necessarily if any $y$ has $G$, it has $F$

SSS $=$ Supervenience as Satisfactorily Supported

Th: MPS in its full generality (i.e. without any restriction on the mental differences which are meant to entail physical differences) can be satisfactorily supported if and only if one is willing to make any of some substantial assumptions about the nature of mental and physical properties

WA1 $=1^{\text {st }}$ Wayward-Atom-Desideratum: Given a relation of co-variance between mental and physical properties, the physical differences which are entailed by large mental differences must themselves be large

WA2 $=2^{\text {nd }}$ Wayward-Atom-Desideratum: Given a relation of co-variance between mental and physical properties, the physical differences which are entailed by mental differences must be differences which are relevant to the mental differences 
WA3 $=3^{\text {rd }}$ Wayward-Atom-Desideratum: To have a relation of psychophysical dependence, we must have, as relata, specific sets of mental and physical properties; the whole families won't do

WGS = Weak forms of Global Supervenience, i.e. forms of global supervenience which require only that there be some mapping under which $A$ indiscernibility is yielded by $B$-indiscernibility

W/S = The distinction between Weak and Strong supervenience

WS1: A set of properties $A$ weakly supervenes on a set of properties $B$ iff for any world $w$, and for any objects $x$ and $y$, if $x$ and $y$ are $B$-indiscernible in $w$, then they are $A$-indiscernible in $w$

WS2: A set of properties $A$ weakly supervenes on a set of properties $B$ iff necessarily for any object $x$ and for any property $F$ in $A$, if $x$ has $F$, then there exists a property $G$ in $B$ such that $x$ has $G$, and if any $y$ has $G$ it has $F$ 